\author{
UNIVERSIDADE DE SÃO PAULO \\ INSTITUTO DE FÍSICA DE SÃO CARLOS
}

Sálvio Jacob Bereta

Vórtices em condensados de Bose-Einstein com geometria esférica

São Carlos

2019 

Sálvio Jacob Bereta

\section{Vórtices em condensados de Bose-Einstein com geometria esférica}

Dissertação apresentada ao Programa de PósGraduação em Física do Instituto de Física de São Carlos da Universidade de São Paulo, para obtenção do título de Mestre em Ciências.

Área de concentração: Física Teórica e Experimental

Orientadora: Dra. Mônica Andrioli Caracanhas Santarelli

\section{Versão corrigida}

(versão original disponível na Unidade que aloja o Programa)

\section{São Carlos}


AUTORIZO A REPRODUÇÃO E DIVULGAÇÃO TOTAL OU PARCIAL DESTE TRABALHO, POR QUALQÜER MEIO CONVENCIONAL OU ELETRÔNICO PARA FINS DE ESTUDO E PESQUISA, DESDE QUE CITADA A FONTE.

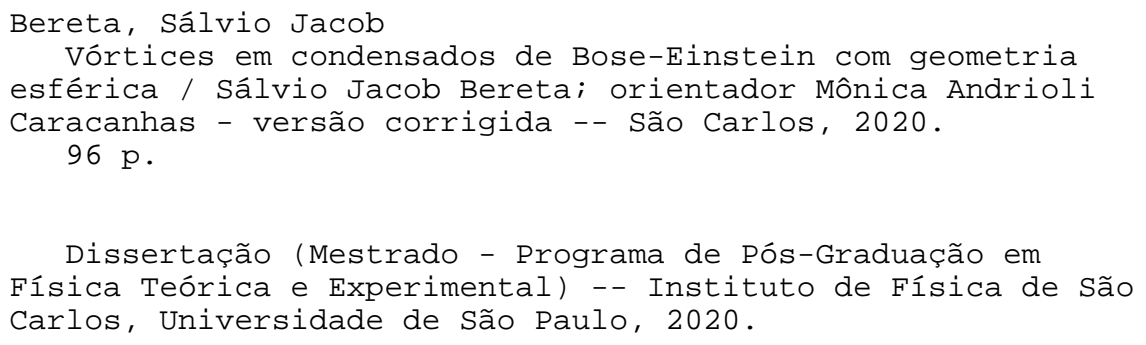

1. Condensado de Bose-Einstein. 2. Bubble Trap. 3. Densidade de Estados. 4. Pares de Vórtices. 5. Dipolos. I. Caracanhas, Mônica Andrioli, orient. II. Título. 


\section{AGRADECIMENTOS}

Gostaria de agradecer minha orientadora Dr. Mônica Andrioli Caracanhas Santarelli pela orientação do meu mestrado e desta dissertação, por todas reuniões em que me ensinou o conteúdo necessário para poder desenvolver a pesquisa, e por tanta paciência em corrigir meus erros. Muito obrigado, minha amiga Mônica.

Agradeço as pessoas que me ajudaram a desenvolver esse trabalho, em especial ao Pós-doutorando Lucas Madeira por suas sugestões e por me ajudar na parte de programação, e ao Prof. Dr. Vanderlei Salvador Bagnato pelas suas ideias criativas. Agradeço a eles, juntamente a minha orientadora, pelas reuniões em grupo muito úteis para o desenvolvimento da pesquisa.

Agradeço aos meus pais: Saula Maria Jacob Bereta e José Gabriel Bereta, pelo apoio amoroso e emocional, pelos ensinamentos que me fizeram alcançar meus objetivos como pessoa e profissional. E também a toda minha família que me incentivou e me apoiou quando mais precisava.

Agradeço aos meus amigos colegas do IFSC da sala 20 que me ajudaram sanando minhas dúvidas quando eu os procurava.

Agradeço ao Prof. Dr. Alexander L. Fetter pelo auxílio no desenvolvimento do capítulo 5 desta dissertação.

"O presente trabalho foi realizado com apoio da Coordenação de Aperfeiçoamento de Pessoal de Nível Superior - Brasil (CAPES) - Código de Financiamento 001". 



\section{RESUMO}

\section{BERETA, S. J. Vórtices em condensados de Bose-Einstein com geometria esfé-}

rica. 2019. 96p. Dissertação (Mestrado em Ciências) - Instituto de Física de São Carlos, Universidade de São Paulo, São Carlos, 2019.

Neste texto será apresentada uma introdução ao condensado de Bose-Einstein ${ }^{1-3} \mathrm{em}$ armadilhas com simetria esférica, também conhecida como "bubble trap", que consiste da caixa e casca esféricas ${ }^{4,5}$, seguido de um estudo sobre a estabilidade e dinâmica de vórtices nessa configuração do condensado. Para averiguarmos a ocorrência da condensação nessa geometria, calculamos o cumulante e a densidade de estados, usando cálculo numérico ${ }^{6} \mathrm{e}$ aproximação analítica (semi-clássica) ${ }^{7,8}$. A diferença nos resultados de ambos os métodos, quando aplicados nas diferentes configurações de armadilha, revelou como as condições de contorno afetam os auto-estados do problema quântico, e consequentemente o valor da temperatura crítica de condensação. Na segunda parte do trabalho determinamos a dinâmica de vórtices em filme superfluido na casca esférica fina (duas dimensões), respeitando o vínculo da superfície fechada ${ }^{9}$ quanto à neutralidade total das cargas ${ }^{10,11}$, isto é, considerando apenas configurações com pares de vórtices de circulação oposta. Derivamos o campo de velocidade na superfície da esfera com cálculo do potencial de fluxo ${ }^{12}$, aplicando a transformação conforme e projeção estereográfica ${ }^{13}$ : após calcular o potencial de um par de vórtices no plano complexo ${ }^{12}$, determinamos como seria esse mesmo potencial na superfície da esfera com a transformação de coordenadas relativa à projeção estereográfica $^{13,14}$. Determinamos também a energia total do sistema, obtendo uma expressão simplificada da energia de interação entre os vórtices em termos do potencial de fluxo. Verificamos a instabilidade dos pares, e a ocorrência da aniquilação dos vórtices na presença de dissipação. Por fim, utilizamos a aproximação de dipolo para derivar a energia de interação entre os dipolos, a qual varia como função do alinhamento dos dipolos. Através dessa energia pudemos analisar a estabilidade e prever a dinâmica dos dipolos na superfície da esfera.

Palavras-chave: Condensado de Bose-Einstein. Bubble Trap. Densidade de Estados . Pares de Vórtices. Dipolos. 



\section{ABSTRACT}

BERETA, S. J. Vortices in Bose-Einstein condensate on spherical geometry. 2019. 96p. Dissertação (Mestrado em Ciências) - Instituto de Física de São Carlos, Universidade de São Paulo, São Carlos, 2019.

In this text it is presented an introduction of the Bose-Einstein condensate ${ }^{1-3}$ in a spherical symmetric trap, known as "bubble trap", which consists in a spheric box and shell ${ }^{4,5}$, followed by a study of the stability and dynamics of vortices in this configuration. In order to check the occurrence of condensation in this geometry we calculated the cumulant and density of state, using numerical calculation ${ }^{6}$ and analytical approximation (semi-classical) ${ }^{7,8}$. The difference between the results in both methods, when applied in different configurations of the trap, revealed how the boundary conditions affects the eingenstates of the quantum problem, and hence the value of the critical temperature of condensation. In the second part of the text we determine the dynamics of vortices in a superfluid bubble-shaped film (two dimensions), respecting the charge neutrality ${ }^{10,11}$ constraint imposed by this closed surface, in other words, considering only the configurations with pairs of vortices with opposite circulations. We derive the velocity field on the surface of the sphere, we calculated the flow potential ${ }^{12}$ by using a conformal transformation and stereographic projection: after calculating the potential of a vortex pair in the complex plane ${ }^{12}$, we determined how it would be the same flow potential in the surface of sphere by using the coordinates transformation of the stereoghaphic projection ${ }^{13,14}$. We also determinated the total energy of the system, obtaining a simplified expression to the interacting energy between the vortices in terms of the flow potential. We verified the instability of pairs and the vortices anihilation in the presence of dissipation. Finally, we apply the dipole approximation to derive the interaction energy between the dipoles, which varies with their alignment. Through this energy we could analyse the instability and predict the dipole dynamics on the surface.

Keywords: Bose-Einstein Condensate. Bubble Trap. Density of States. Vortexs pairs. Dipoles. 



\section{LISTA DE FIGURAS}

Figura 1 - Representação da evolução do comprimento de onda térmico de De Broglie para diferentes temperaturas decrescentes.

Fonte: Elaborada pelo autor. . . . . . . . . . . . . . . . . . . . . . . . 19

Figura 2 - Um quarto de anel de raio $n$ representando uma classe de partículas $\operatorname{com}$ energia $\varepsilon$.

Fonte: Elaborada pelo autor. . . . . . . . . . . . . . . . . . . . . . 23

Figura 3 - Curvas das três primeiras funções esféricas de Bessel e de Neumann. Fonte: Elaborada pelo autor. . . . . . . . . . . . . . . . . . . . 27

Figura 4 - Gráfico do cumulante $G(\varepsilon)$ em função de $\varepsilon$ para $0 \leq \varepsilon \leq 12000 \varepsilon_{0}$, onde $\varepsilon_{0} \frac{\hbar^{2}}{2 m a^{2}}$ e $a=10^{-5} \mathrm{~m}$.

Fonte: Elaborada pelo autor. . . . . . . . . . . . . . . . . . . 28

Figura 5 - Representação da razão entre a diferença dos valores dos cumulantes da caixa esférica obtidos pelas raizes das equações de Bessel e pela aproximação semi-clássica e o próprio cumulante obtido pelas raizes das equações de Bessel.Observe que: $C_{s}$ é o cumulante obtido pela aproximação semi-clássica e $C_{q}$ é o cumulante da caixa obtido pelos autovalores do sistema.

Fonte: Elaborada pelo autor. . . . . . . . . . . . . . . . . . . . . 29

Figura 6 - Representação gráfica de $\ln [G(\varepsilon)]$ por $\ln (\varepsilon)$ para $0 \leq \varepsilon \leq 12000 \varepsilon_{0}$, com intervalo de energia de $1000 \varepsilon_{0}$ entre cada ponto.

Fonte: Elaborada pelo autor. . . . . . . . . . . . . . . . . . . . 30

Figura 7 - Representação dos coeficientes angulares e lineares (intervalos de energia $\sim 10^{3}$ ), vemos que os pontos tendem a linha tracejada (valor dos coeficientes da aproximação semi-clássica) conforme $\varepsilon$ aumenta para diferentes intervalos de momento linear apresentados no quadro.

Fonte: Elaborada pelo autor. . . . . . . . . . . . . . . . . . . .

Figura 8 - Temperaturas críticas calculadas com os coeficientes obtidos pelo cumulante calculado numericamente para raio $a=10^{-5} \mathrm{~m}$ comparado ao modelo semi-clássico.

Fonte: Elaborada pelo autor. . . . . . . . . . . . . . . . . . . . 32

Figura 9 - Temperaturas críticas calculadas com os coeficientes obtidos pelo cumulante calculado numericamente para raio $a=1,33 \times 10^{-5} \mathrm{~m}$ comparadas para raio $a=10^{-5} \mathrm{~m}$.

Fonte: Elaborada pelo autor. . . . . . . . . . . . . . . . . . 33 
Figura 10 - Cumulantes da caixa e da casca esféricas no regime quântico e semiclássico.

Fonte: Elaborada pelo autor. . . . . . . . . . . . . . . . . . . . . . . 34

Figura 11 - Representação dos coeficientes angulares e lineares das funções $\ln [G(\varepsilon)]$ por $\ln (\varepsilon)$ para diferentes raio internos $a$, onde $\delta R=(b-a)$.

Fonte: Elaborada pelo autor. . . . . . . . . . . . . . . . . . . 35

Figura 12 - Temperaturas críticas para diferentes raios que formam a casca esférica. Fonte: Elaborada pelo autor. . . . . . . . . . . . . . . . . . 36

Figura 13 - Comparação de temperaturas críticas de mesmo volume mas diferentes formatos (caixa e casca).

Fonte: Elaborada pelo autor. . . . . . . . . . . . . . . . . . . 36

Figura 14 - Esquema de um vórtice precessionando em uma nuvem armadilhada com rotação $\Omega$. O vórtice rotaciona com velocidade angular $w_{a}$ enquanto que o centro de massa (CM)do condensado também rotaciona, mas com diferença de fase de $\pi$.

Fonte: Elaborada pelo autor. . . . . . . . . . . . . . . . . 57

Figura 15 - Localização de certo vórtice $i$ na posição $r_{i}$ em um ângulo $\phi_{i}$ atuando em certo ponto determinado por $r$ e $\phi$ a uma distância $d_{i}$.

Fonte: Elaborada pelo autor. . . . . . . . . . . . . . . . . . 58

Figura 16 - A e B correspondem a vórtices de vorticidades iguais e opostas.

Fonte: Elaborada pelo autor. . . . . . . . . . . . . . . . . . . . . . . 59

Figura 17 - Dinâmica de um vórtice e um anti-vórtice, onde (a) são imagens experimentais e (b) imagens de simulação numérica.

Fonte: NEELY et $a l^{15} \ldots \ldots$. . . . . . . . . . . . . . . . . . 61

Figura 18 - Linhas de velocidade de fluxo de dois vórtices de sinais opostos e separados de $\pi, \vec{n}$ é a normal.

Fonte: Elaborada pelo autor. . . . . . . . . . . . . . . . . . .

Figura 19 - Projeção estereográfica da esfera no plano. Na figura podemos observar a projeção de um ponto qualquer $\mathrm{P}$.

Fonte: Elaborada pelo autor. . . . . . . . . . . . . . . . . . . .

Figura 20 - Par de vórtices em uma posição qualquer enquanto que os eixos adotados são tais que ambos estão em $\phi=0$ e $\theta=\pi / 2$ coincide com o ponto médio entre eles.

Fonte: Elaborada pelo autor. . . . . . . . . . . . . . . . .

Figura 21 - As situações propostas A e B esquematizam os dipolos de vórtices dispostos simetricamente e antisimetricamente respectivamente.

Fonte: Elaborada pelo autor. . . . . . . . . . . . . . . . . . . . . . . 70 
Figura 22 - Representação de dipolos alinhados (situação A) em que o primeiro vórtice está localizado em $(\theta, \phi)=(0,0)$. No caso em que o segundo dipolo inverta teremos a situação B.

Fonte: Elaborada pelo autor. . . . . . . . . . . . . . . . . . . 73 



\section{LISTA DE ABREVIATURAS E SIGLAS}

$\begin{array}{ll}\text { BEC } & \text { Bose-Einstein Condensate } \\ \text { GPE } & \text { Gross-Pitaevskii Equation } \\ \text { IFSC } & \text { Instituto de Física de São Carlos } \\ \text { TF } & \text { Thomas-Fermi } \\ \text { USP } & \text { Universidade de São Paulo }\end{array}$





\section{LISTA DE SÍMBOLOS}

\begin{tabular}{|c|c|}
\hline$\alpha$ & Constante genérica \\
\hline$\beta$ & Um dividido por constante de Boltzmann vezes a temperatura \\
\hline$\gamma$ & Ângulo entre o vórtice e o ponto de interesse na casca esférica \\
\hline$\delta$ & Ângulo do desalinhamento dos dipolos de vórtices \\
\hline$\epsilon$ & Abertura angular dos dipolos na casca \\
\hline$\varepsilon$ & Autovalor de energia \\
\hline$\eta$ & Deslocamento do centro de massa da nuvem \\
\hline$\theta$ & Ângulo raidial entre o eixo z e o ponto de interesse \\
\hline$\lambda$ & Comprimento de onda \\
\hline$\mu$ & Potencial químico \\
\hline$\xi$ & "Core" do vórtice \\
\hline$\pi$ & Razão entre a circunferência e o diâmetro de um disco \\
\hline$\rho$ & Coordenada polar \\
\hline$\tau$ & Número de partículas por unidade de comprimento em z \\
\hline$\phi$ & Coordenada angular \\
\hline$\chi$ & Função de fluxo \\
\hline$\psi$ & Função de onda de uma partícula \\
\hline$\zeta$ & Função zeta de Riemman \\
\hline$\Delta$ & Variação angular \\
\hline$\Gamma$ & Contorno em superfície fechada \\
\hline$\Pi$ & Produtorial \\
\hline$\Sigma$ & Somatório \\
\hline$\Psi$ & Função de onda \\
\hline$<$ & Menor \\
\hline
\end{tabular}




\begin{tabular}{|c|c|}
\hline$>$ & Maior \\
\hline$=$ & Igual \\
\hline$\leq$ & Menor ou igual \\
\hline$\geq$ & Maior ou igual \\
\hline$\ll$ & Muito menor \\
\hline$\gg$ & Muito maior \\
\hline$\approx$ & Aproximadamente \\
\hline$\sim$ & Da magnitude \\
\hline$\|$ & Módulo \\
\hline $\int$ & Integral \\
\hline$\oint$ & Integral de caminho \\
\hline$\frac{\mathrm{d}}{\mathrm{d} x}$ & Derivda total com respeito a $x$ \\
\hline$\frac{\partial}{\partial x}$ & Derivada parcial com respeito a $x$ \\
\hline$\nabla^{2}$ & Laplaciano \\
\hline$\nabla$ & Divergente \\
\hline$\nabla$ & Gradiente \\
\hline$\dot{r}$ & Derivada de $r$ \\
\hline$x$ & Produto vetorial \\
\hline . & Produto escalar \\
\hline$\rightarrow$ & Tende a \\
\hline
\end{tabular}




\section{SUMÁRIO}

INTRODUÇÃo $\ldots \ldots \ldots \ldots \ldots \ldots$

MECÂNICA ESTATÍSTICA DOS BÓSONS . . . . . . . . . . . . 21

2.1.1 Temperatura crítica do Condensado de Bose-Einstein . . . . . . . . . . . 24

2.2 Densidade de Estados em uma Caixa Esférica . . . . . . . . . . 25

$2.2 .1 \quad$ Método numérico para cálculo do cumulante na caixa esférica . . . . . . 27

2.2.2 Cálculo da Temperatura crítica na caixa esférica . . . . . . . . . . . 30

2.3 Densidade de Estados em uma Casca Esférica . . . . . . . . . . . 31

2.3.1 Método numérico para cálculo do cumulante na caixa esférica . . . . . . 33

2.3.2 Cálculo da temperatura crítica na casca esférica . . . . . . . . . . 35

2.3.3 Casca puramente $2 \mathrm{D} \ldots \ldots \ldots \ldots \ldots \ldots$

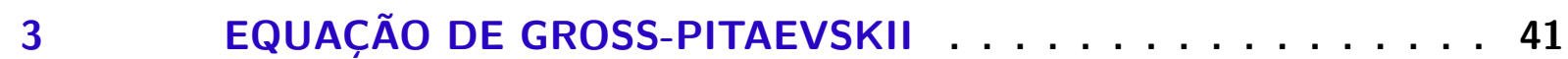

3.1 Introdução da Equação de Gross-Pitaevskii. . . . . . . . . . . . 41

3.2 Equação de Gross-Pitaevskii dependente do tempo e relações com a dinâmica de fluidos . . . . . . . . . . . . . . 46

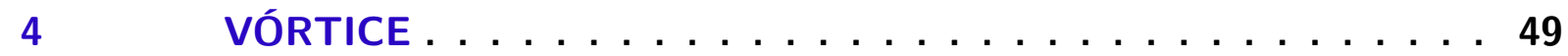

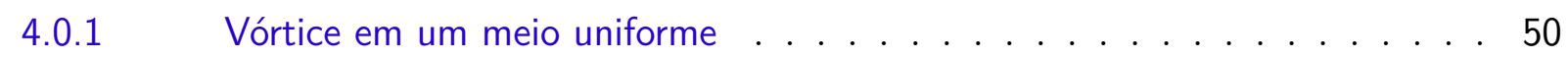

4.0.2 Vórtice com muitos quanta de energia . . . . . . . . . . . . . 52

4.0.3 Vórtice em uma nuvem armadilhada . . . . . . . . . . . . . . 53

4.0.4 Armadilhas que Rotacionam . . . . . . . . . . . . . . . . . 54

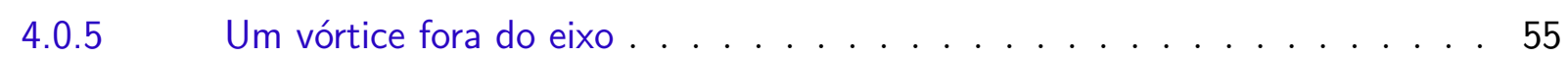

4.1 Dinâmica de um vórtice armadilhado . . . . . . . . . . 56

4.2 Interação de um par de vórtices . . . . . . . . . . . . . 57

$4.2 .1 \quad$ Efeito Magnus . . . . . . . . . . . . . . . . . . . . . . . . 59

4.3 Dinâmica Combinada de Interação Vórtice-Nuvem e Par de Vórtices 60

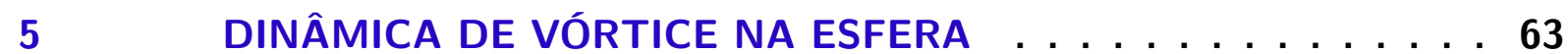

5.1 Projeção estereográfica para dois vórtices na esfera . . . . . . 65

$5.2 \quad$ Energia de interação . . . . . . . . . . . . . 67

5.3 Dinâmica de um par de vórtice na casca esférica . . . . . . 68

5.3.1 Dinâmica de dois pares de vórtices na esfera . . . . . . . . . . . . . 69

5.4 Minimização de energia de dipolos . . . . . . . . . . . . 72

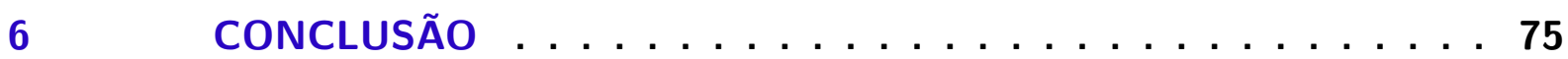




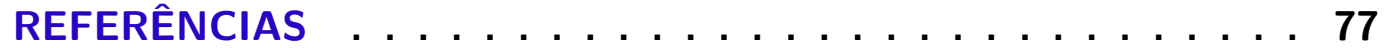

APÊNDICES

APÊNDICE A - DEMONSTRAÇÃO DO RESULTADO DA INTEGRAL UTILIZADO PARA CONCLUIR A EQ. 2.383

APÊNDICE B - DENSIDADE DE ESTADOS DO OSCILADOR HARMÔNICO ........... . 85

APÊNDICE C - DENSIDADE DE ESTADOS DO OSCILADOR HARMÔNICO UTILIZANDO A EQ. 2.3 . . . 87

APÊNDICE D - DEMONSTRAÇÃO DO RESULTADO DA INTEGRAL UTILIZADO PARA CONCLUIR A EQ. 2.1689

APÊNDICE E - DEMONSTRAÇÃO DAS EQ. 2.22 E $2.24 \ldots \ldots 91$

APÊNDICE F- DEDUÇÃO DA EQ. $2.48 \ldots \ldots \ldots$

APÊNDICE G - DEDUÇÃO DA EQ. $5.13 \ldots \ldots \ldots$ 


\section{INTRODUÇÃO AO CONDENSADO DE BOSE-EINSTEIN}

Um condensado de Bose-Einstein (BEC) consiste na ocupação macroscópica do estado de partícula única de mais baixa energia, ou seja, no estado de mais baixa energia (veja Refs. ${ }^{16-18}$ para uma visão mais ampla da história). O BEC foi inicialmente previsto por Einstein em 1925, utilizando um método estatístico introduzido por Satyendra Nath Bose. Segundo o artigo publicado por Einstein ${ }^{2}$, quando um gás de bósons for resfriado abaixo de certa temperatura crítica $\left(T_{c}\right)$, uma fração das partículas irá para o estado fundamental e formará um novo estado da matéria, diferente daqueles que já conhecemos (sólido, líquido e gasoso) ${ }^{1}$.

Considerando as partículas descritas por uma função de onda, podemos associar a elas o comprimento de onda térmico de De Broglie $\lambda_{d B}=\sqrt{2 \pi \hbar^{2} / m k_{B} T}$, onde $\hbar$ corresponde à constante de Planck dividida por $2 \pi, k_{B}$ é a constante de Boltzmann, $m$ é a massa da partícula e $T$ a temperatura do sistema. Uma característica desse novo estado da matéria é que $\lambda_{d B}$ se torna da ordem da distância média entre as partículas de forma que, para temperaturas $T$ suficientemente baixas, haverá a sobreposição da função de onda dos átomos, que se tornam indistinguíveis conforme esquematizado na Fig. 1, Ref. ${ }^{3}$.

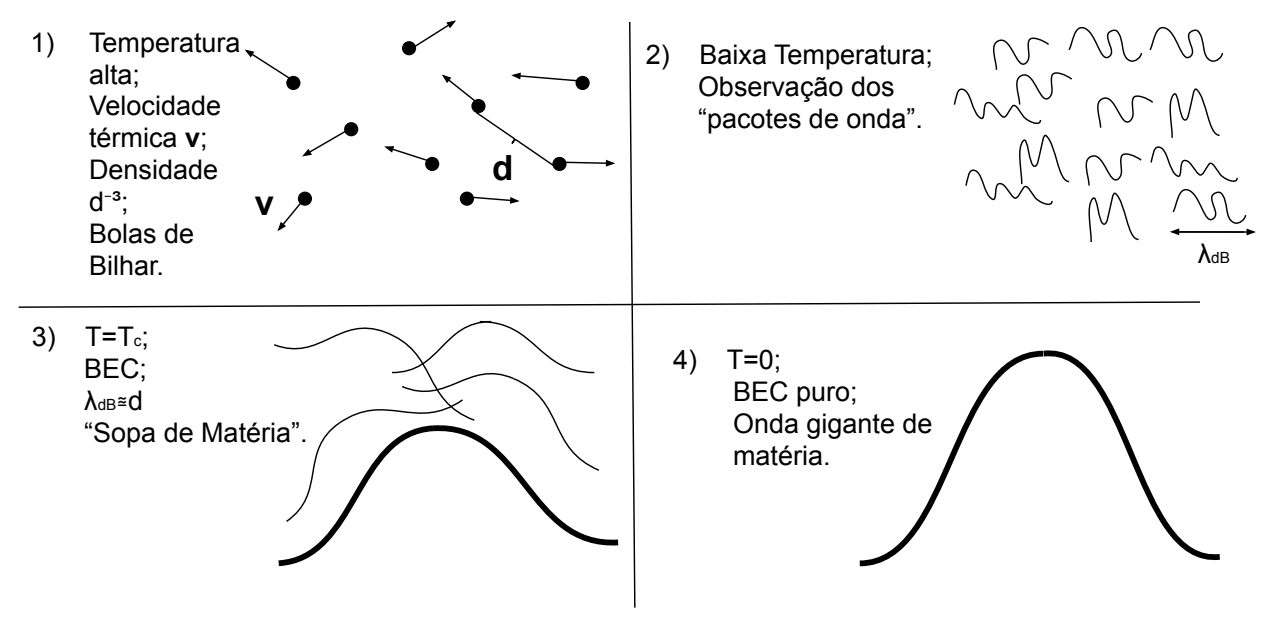

Figura 1: Representação da evolução do comprimento de onda térmico de De Broglie para diferentes temperaturas decrescentes.

Fonte: Elaborada pelo autor.

Uma estimativa da temperatura crítica de condensação $T_{c}$ pode ser dada pela relação $n \lambda_{d B}^{3}=2,612^{7}$, onde a distância média entre os átomos é dada em termos da densidade do gás $n\left(d \sim 1 / n^{1 / 3}\right)^{3}$. Por exemplo, para o ${ }^{4} \mathrm{He}$, cuja densidade típica de $n \sim 10^{20} \mathrm{~m}^{-3}{ }^{3}$, a temperatura crítica $T_{c}$ é da ordem de $1 \mathrm{~K}{ }^{19}$, enquanto que gases bosônicos 
de metais alcalinos (como ${ }^{7} \mathrm{Li},{ }^{23} \mathrm{Na}$ e ${ }^{87} \mathrm{Rb}$ ) possuem $T_{c}$ da ordem de $100-1000 \mathrm{nK}{ }^{3}$, em razão da maior massa atômica e do baixo valor de densidade $n \sim 10^{13} \mathrm{~cm}^{-3}=10^{19} \mathrm{~m}^{-3}$. Não há formação de BEC em superfícies planas de duas dimensões, ver Ref. ${ }^{20}$.

A grande motivação de se estudar o BEC é desvendar as propriedades dessa nova matéria quântica macroscópica. Vale ressaltar que a primeira observação experimental de um BEC ${ }^{21-23}$ foi feita em 1995 por Eric A. Cornell; Wolfgang Ketterle and Carl E. Wieman, vencedores do prêmio Nobel em 2001, depois de inúmeras tentativas para resfriar átomos da primeira coluna da tabela periódica ${ }^{3}$.

O BEC se forma através da saturação do estado de ocupação das partículas excitadas $^{1}$. Quando um sistema não comporta nenhuma outra partícula excitada, toda partícula adicionada irá para estado fundamental. Esse aglomerado de partículas no estado fundamental é o que denominamos BEC. Para contabilizarmos o número de partículas excitadas possíveis devemos considerar a probabilidade de uma partícula estar em certo estado de energia (função de Bose) vezes a quantidade de estados dessa mesma energia, como será abordado no Capítulo 2, onde tentamos validar a existência do BEC armadilhado na caixa e casca esféricas. Esse estudo de um BEC armadihado é motivado pela possibilidade experimental de confinar átomos neste tipo de armadilha ${ }^{4,5,24}$, a qual deve estar inserida em microgravidade para produzir uma distribuição atômica esférica (caso contrário as partículas serão puxadas pela gravidade para o polo sul) ${ }^{25}$. Vale observar que existe uma proposta ${ }^{26}$ para realização de um experimento para gerar um BEC casca esférica usando um aparato experimental em órbita (NASA Cold Atom Laboratory, a bordo da International Space Station).

Quando ocore uma perturbação no BEC de forma que ele adquira momento angular, haverá formação de vórtices quantizados. Trata-se de uma singulariadade local da função de onda macroscópica do BEC, cujo campo de velocidade se associa a um número inteiro do quantum de circulação ${ }^{1}$. Enquanto os vórtices clássicos assumem qualquer valor de circulação, os superfluidos são irrotacionais, e qualquer rotação ou momento angular é restrita a ocorrer através de vórtices com circulação quantizada. O vórtice quântico ${ }^{27}$ será discutido no Capítulo 3. Esses vórtices são formados somente quando a armadilha é rotacionada em uma frequência maior do que um certo valor crítico, conforme será apresentado no Capítulo 4. Na sequência, analisaremos no Capítulo 5 a dinâmica de vórtice na casca esférica. Finalmente no Capítulo 6 apresentamos nossas conclusões e perspectivas para a continuação desta pesquisa 


\section{MECÂNICA ESTATÍSTICA DOS BÓSONS}

O sistema de interesse é assumido estar em equilíbrio com um reservatório de temperatura $T$ e potencial químico $\mu$, o qual determina a energia média e o número médio de partículas do sistema. Considerando um gás ideal de bósons, as propriedades termodinâmicas desse sistema podem ser obtidas através da função de partição grancanônica $^{28}$ :

$$
\ln \Omega(T, V, \mu)=-\sum_{j} \ln \left(1-e^{-\beta\left(\varepsilon_{j}-\mu\right)}\right)
$$

onde $\beta=1 / k_{B} T$ e $\varepsilon_{j}$ é a energia do estado $j$, com índice $j$ varrendo todos os possíveis autoestados do sistema. Através das relações termodinâmicas ${ }^{28}$ podemos obter a probabilidade de uma partícula ocupar certo estado de energia $\varepsilon, N_{B}(\varepsilon)$, também conhecida como distribuição de Bose-Einstein, a qual será proporcional à derivada da função de partição gran-canônica em relação à energia:

$$
N_{B}(\varepsilon)=-\frac{1}{\beta} \frac{\partial \ln \left(1-e^{-\beta(\varepsilon-\mu)}\right)}{\partial \varepsilon}=\frac{1}{e^{\beta(\varepsilon-\mu)}-1} .
$$

Para que a função de ocupação assuma valores estritamente positivos, temos sempre que garatir que $\varepsilon>\mu$, com $\mu$ estando associado à energia do estado fundamental. É também conveniente definir a fugacidade do sistema pelo fator $z=e^{-\beta \mu}$, o qual assume valor igual a 1 no BEC ideal, $\mu=0$ (uma explicação mais clara sobre o conceito do potencial químico em um contexto mais amplo pode ser obtida na Ref. ${ }^{29}$ ), porque o custo energético para adicionar uma partícula no estado fundamenal é nulo.

\subsection{Densidade de Estados}

O conceito de densidade de estados é empregado em muitas áreas da física, como por exemplo no cálculo de taxas de reação nuclear, do calor específico, da radiação de corpo negro e também no cálculo da temperatura crítica do BEC. Para defirmos a densidade de estados $g(\varepsilon)$, devemos contabilizar o número de estados contidos no intervalo de energia $\varepsilon$ e $\varepsilon+d \varepsilon$ (para uma visão mais abrangente e pedagógica sobre densidade de estados, ver Ref. $\left.{ }^{30}\right)$.

Começando com a análise semi-clássica, podemos assumir que o volume no espaço de fase delimitado pelas superfícies de energia $\varepsilon$ e $\varepsilon+d \varepsilon$ é proporcional ao número de estados dentro desse intervalo de energia ${ }^{7,8}$. Em particular, a expressão semi-clássica para densidade de estados pode ser generalizada para o sistema com potencial externo $U(\mathbf{r})$, de 
forma que a densidade de estados será dada por (ver apêndice A):

$$
g(\varepsilon)=\frac{2 \pi(2 m)^{3 / 2}}{h^{3}} \int_{V(\varepsilon)} \sqrt{\varepsilon-U(\mathbf{r})} d^{3} r
$$

onde $V(\varepsilon)$ é o volume do espaço de fase disponível para as partículas com energia $\varepsilon$. Introduziremos também o conceito de cumulante, $G(\varepsilon)$. O cumulante é a função que nos dá o número de estados de energia até um certo valor $\varepsilon$ de energia do sistema. É facil concluir que a sua derivada deverá corresponder a densidade de estados do sistema, isto é, $g(\varepsilon)=\mathrm{d} G(\varepsilon) / \mathrm{d} \varepsilon$. Agora vamos solucionar $g(\varepsilon)$ e $G(\varepsilon)$ para o caso onde as partículas estão livres, $U(\mathbf{r})=0$, temos que

$$
g(\varepsilon)=\frac{V}{(2 \pi)^{2}}\left(\frac{2 m}{\hbar^{2}}\right)^{3 / 2} \sqrt{\varepsilon}
$$

e, consequentemente,

$$
G(\varepsilon)=\frac{2 V}{3(2 \pi)^{2}}\left(\frac{2 m}{\hbar^{2}}\right)^{3 / 2} \varepsilon^{3 / 2}
$$

Perceba que o cálculo da densidade semi-clássica e do cumulante semi-clássico indenpende do formato do confinamento, mas apenas do valor do volume, ou seja, as partículas não sentem as paredes que delimitam o confinamento nesse modelo.

Um dos sistemas mais simples de serem resolvidos para o cálculo da densidade de estados seria o da partícula em uma caixa. No caso unidimensional de uma caixa de tamanho L, os níveis de energia são dados por ${ }^{31}$ :

$$
\varepsilon=n^{2} \varepsilon_{0}
$$

onde $\varepsilon_{0}=\pi^{2} \hbar^{2} /\left(2 M L^{2}\right)$ com $n$ um número inteiro $(1,2,3 \ldots)$ que rotula os autoestados. Através do espectro de energia é possível achar o número de estados quânticos com energia menor do que $\varepsilon$ (cumulante em uma dimensão $G_{1}$ ) como função da energia. Por exemplo, para $\varepsilon=81 \varepsilon_{0}$ temos 9 estados possíveis $\left(\varepsilon_{0} ; 2^{2} \varepsilon_{0} ; 3^{2} \varepsilon_{0} \ldots 9^{2} \varepsilon_{0}\right)$, ou seja, o cumulante será $G_{1}\left(81 \varepsilon_{0}\right)=9$. Por indução, considerando o caso contínuo, onde os intervalos de energias são pequenos comparados à energia total do sistema, o cumulante será $G(\varepsilon)=\left(\varepsilon / \varepsilon_{0}\right)^{1 / 2} \mathrm{e}$ a densidade de estados em uma dimensão será:

$$
g_{1}(\varepsilon)=\frac{\mathrm{d} G_{1}(\varepsilon)}{\mathrm{d} \varepsilon}=\frac{1}{2 \sqrt{\varepsilon \varepsilon_{0}}} .
$$

Já no caso 2D (quadrado de lado L) a energia pode ser obtida através de $\varepsilon=$ $\left(n_{x}^{2}+n_{y}^{2}\right) \varepsilon_{0}$, onde $n_{x}$ e $n_{y}$ são números inteiros positivos. Se representarmos em um gráfico a energia $\varepsilon$ como função de $n_{x}$ e $n_{y}$, conforme mostra a Fig. 2, a distância entre as coordenadas $(0,0)$ e $\left(n_{x}, n_{y}\right)$ representará uma classe de estados com energia $\varepsilon=n^{2} \varepsilon_{0}$, onde $n^{2}=n_{x}^{2}+n_{y}^{2}$. Dessa forma, um quarto da área (somente os eixos positivos $n_{i}>0$ ) entre os raios $\varepsilon$ e $\varepsilon+d \varepsilon$ conterá o número de estados em tal intervalo:

$$
g_{2}(\varepsilon) d \varepsilon=\frac{\pi}{4}\left[(n+d n)^{2}-n^{2}\right]=\frac{\pi}{4 \varepsilon_{0}} d \varepsilon .
$$


Note que na última igualdade utilizamos $n=\sqrt{\varepsilon / \varepsilon_{0}}$.

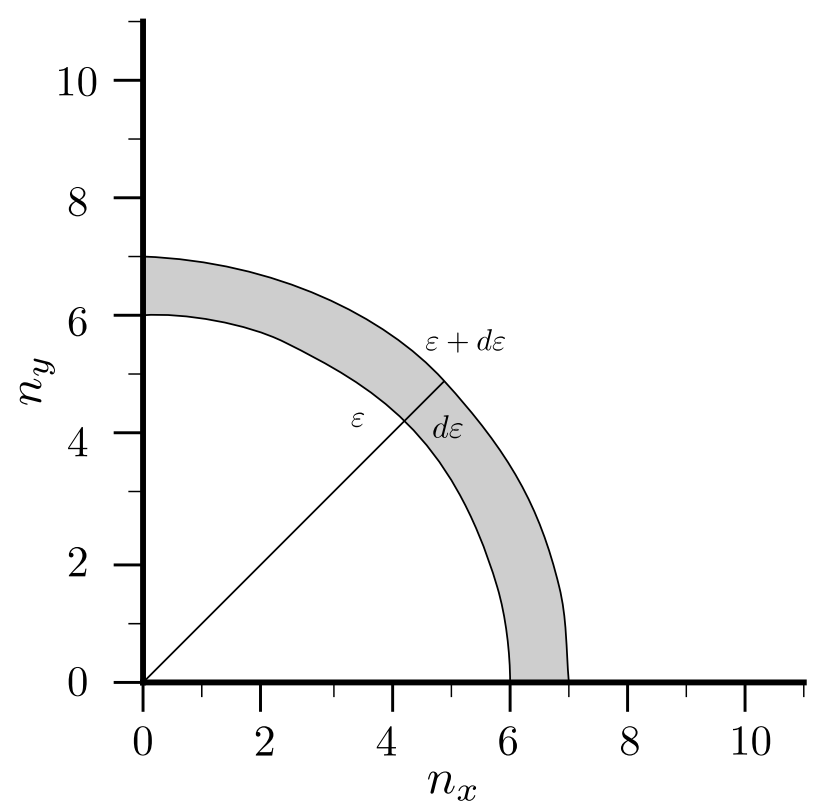

Figura 2: Um quarto de anel de raio $n$ representando uma classe de partículas com energia $\varepsilon$. Fonte: Elaborada pelo autor.

No caso da caixa em três dimensões (cubo de lado L), o espectro de energia do sistema será $\varepsilon=\varepsilon_{0}\left(n_{x}^{2}+n_{y}^{2}+n_{z}^{2}\right)$, com $n_{i}(i=x, y, z)$ inteiros positivos. Podemos escrever a energia na forma $\varepsilon=n^{2} \varepsilon_{0}$, com n sendo o raio no sistema cartesiano com eixos $n_{x}, n_{y}$ e $n_{z} ; n^{2}=n_{x}^{2}+n_{y}^{2}+n_{z}^{2}$. De forma análoga ao caso $2 \mathrm{D}$, a densidade de estados será o volume que compreende um oitavo do volume (somente os eixos positivos $n_{i}>0$ ) da casca esférica entre os raios $\varepsilon$ e $\varepsilon+d \varepsilon$ :

$$
g_{3}(\varepsilon) d \varepsilon=\frac{4 \pi}{24}\left[(n+d n)^{3}-n^{3}\right]=\frac{\pi \sqrt{\varepsilon}}{2 \varepsilon_{0}^{3 / 2}} d \varepsilon
$$

$\operatorname{com} n=\sqrt{\varepsilon / \varepsilon_{0}}$

Pelos resultados obtidos, podemos concluir que a densidade de estados para uma caixa em dimensão $D$ será:

$$
g_{D}(\varepsilon)=C_{\alpha} \varepsilon^{(\alpha-1)},
$$

com $\alpha=D / 2$, sendo $D$ a dimensão da caixa. Observe que para $D=3, g_{3}(\varepsilon)$ é proporcional a $\sqrt{\varepsilon}$, assim como no resultado obtido no regime semi-clássico.

Consideraremos agora o caso de um potencial harmônico, com $V(\mathbf{r})$ descrito da forma:

$$
V(\mathbf{r})=\frac{m w^{2}}{2}\left(z^{2}+y^{2}+x^{2}\right)
$$


onde $x, y$ e $z$ são as posições em coordenadas cartesianas. Temos que o espectro de energia do oscilador dado por:

$$
\varepsilon\left(n_{x}, n_{y}, n_{z}\right)=\hbar w\left(n_{z}+n_{y}+n_{x}\right)+\varepsilon_{0}
$$

onde $n_{i}$ corresponde ao autoestado da coordenada cartesiana $i$ e $\varepsilon_{0}=\frac{3}{2} \hbar w$ é a energia do estado fundamental. A densidade de estados do oscilador harmônico pode ser calculada considerando os níveis de energia degenerados do sistema. O que nos dá ${ }^{32}$ (ver apêndice $\mathrm{B})$ :

$$
g_{o s c}(\varepsilon)=\frac{\varepsilon^{2}}{2(\hbar w)^{3}}+\frac{3}{2} \frac{\varepsilon}{(\hbar w)^{2}}+\mathscr{O}\left(\varepsilon^{0}\right) .
$$

Se considerarmos um regime onde $\varepsilon \gg \hbar w_{i}$ para discretizar os $n_{i}$ e negligenciar o ponto zero de energia $\varepsilon_{0}=\frac{3}{2} \hbar w$, isso porque os intervalos de energia serão muito pequenos quando comparados as escalas de energia de interesse, podemos utilizar a Eq. 2.3 para calcular a densidade de estados do oscilador harmônico no regime clássico (ver apêndice C):

$$
g_{o s c}(\varepsilon)=\frac{\varepsilon^{2}}{2(\hbar w)^{3}}
$$

Quando comparamos as duas densidade de estados dadas pela Eq. 2.13 e Eq. 2.14, vemos que a Eq. 2.3 fornece uma boa aproximação para a densidade de estados no regime em que $\varepsilon \gg \hbar w$, pois assim os termos que diferem entre essas duas equações pouco irão contribuir. Isso nos mostra que nesse regime a aproximação semi-clássica é bem eficaz.

O interesse de resolver o potencial harmônico é devido ao armadilhamento necessário para o esfriamento das partículas no laboratório.

\subsubsection{Temperatura crítica do Condensado de Bose-Einstein}

A probabilidade de uma partícula estar em um certo auto-estado de energia é dada pela Eq. 2.2, e o número de auto-estados naquela energia é dada por $g(\varepsilon) d \varepsilon$. Então o número total de partículas excitadas possíveis será dado pela integral (no limite termodinâmico é possível converter o somatório em integral ${ }^{33}$ ) que segue, considerando todo o intervalo de energia possível:

$$
N_{e x}(T)=\int_{0}^{\infty} g(\varepsilon) N_{B}(\varepsilon) d \varepsilon=\int_{0}^{\infty} C_{\alpha} \varepsilon^{\alpha-1} \frac{1}{e^{\beta(\varepsilon-\mu)}-1}
$$

onde $C_{\alpha}$ é uma constante genérica que acompanha a densidade de estados, cuja forma assumimos como $g(\varepsilon)=C_{\alpha} \varepsilon^{\alpha-1}$. O condensado aparece quando o número total de partículas do sistema, $N$, se iguala ao número de partículas excitadas possíveis $N_{e x}$, com o potencial químico $\mu$ convergindo para o valor zero (BEC ideal). A temperatura no qual isso ocorre é chamada de temperatura crítica $T_{c}$. Assim toda partícula adicionada ao sistema irá 
para o estado fundamental, pois estatisticamente não poderá estar excitada, eis o BEC. Resolvendo a integral da Eq. 2.15 para $T=T_{c}$ (ver apêndice D), teremos:

$$
N=N_{e x}\left(T_{c}\right)=C_{\alpha} \Gamma(\alpha) \zeta(\alpha)\left(k_{B} T_{c}\right)^{\alpha},
$$

onde $\Gamma(\alpha)=\int_{0}^{\infty} X^{\alpha-1} e^{-X} d X$ e $\zeta(\alpha)=\sum_{n=1}^{\infty} n^{-\alpha}$. E se isolarmos $T_{c}$ :

$$
T_{c}=\frac{1}{k_{B}}\left[\frac{N}{C_{\alpha} \Gamma(\alpha) \zeta(\alpha)}\right]^{\frac{1}{\alpha}} .
$$

Perceba que a $T_{c}$ é inversamente proporcional a $\zeta(\alpha)$, a qual diverge para $\alpha \leq 1$ (ou $D \leq 2$ ) implicando em $T_{c} \rightarrow 0$. Então não há a formação do BEC, em temperatura finita, para um sistema uniforme infinito em um espaço de dimensão inferior a três ${ }^{20}$. Isso porque o número de partículas excitadas possíveis será infinito, assim todas as partículas do sistema nunca irão se condensar por mais numerosas que sejam.

Se utilizarmos a densidade de estados semi-clássica (Eq. 2.9) para um sistema 3D, $T_{c}$ será dada por:

$$
T_{c}=\left[\frac{N}{\frac{V}{(2 \pi)^{2}} \Gamma(3 / 2) \zeta(3 / 2)}\right]^{\frac{2}{3}} \frac{\hbar^{2}}{2 m k_{B}},
$$

com $T_{c}$ inversamente proporcional a $V^{2 / 3}$. Na Eq. 2.18, reescrevendo $T_{c}$ como função de $\lambda_{d B}$, reobtemos a relação apresentada na introdução, $\left(n \lambda^{3}=2,612\right)$, onde $n=N / V$.

Na sequência determinaremos as densidades de estados e suas respectivas temperaturas críticas para os potenciais de caixa e casca esféricas.

\subsection{Densidade de Estados em uma Caixa Esférica}

Vamos considerar uma partícula de massa $m$ e energia $E>0$ sujeita ao seguinte potencial:

$$
U(r)=\left\{\begin{array}{cc}
0 & 0 \leq r<a \\
\infty & r \geq a
\end{array},\right.
$$

onde $a$ corresponde ao raio da esfera em que a partícula está confinada. A parte espacial da função de onda satisfaz a equação de Schrödinger independente do tempo:

$$
-\frac{\hbar^{2}}{2 m} \nabla^{2} \Psi(\mathbf{r})+V(r) \Psi(\mathbf{r})=E \Psi(\mathbf{r}) .
$$

A simetria do potencial $V(r)$ sugere a adoção de coordenadas esféricas $\mathbf{r} \equiv(r, \theta, \varphi)$ na Eq. 2.20. Considerando separação de variáveis, iremos procurar soluções da forma ${ }^{13}$ :

$$
\Psi_{n, l, m}(r, \theta, \varphi)=R_{n, l}(r) Y_{l, m}(\theta, \varphi) .
$$

Obviamente a função de onda deve ser zero para $r \geq a$ onde o potencial é infinito, e uma função bem comportada para $r=0$. Se aplicarmos Eq. 2.21 na Eq. 2.20, para região 
$0 \leq r<a$, encontramos a equação para a parte radial:

$$
\frac{\mathrm{d}^{2} R_{n, l}}{\mathrm{~d} r^{2}}+\frac{2}{r} \frac{\mathrm{d} R_{n, l}}{\mathrm{~d} r}+\left(k^{2}-\frac{l(l+1)}{r^{2}}\right) R_{n, l}=0,
$$

onde $l$ é um número inteiro positivo e o número de onda $k$ está relacionando com a energia $E$ da partícula através da seguinte equação:

$$
k^{2}=\frac{2 m \varepsilon}{\hbar^{2}} .
$$

Para a parte angular teremos a equação:

$$
\frac{1}{\sin \theta} \frac{\partial\left(\sin \theta \frac{\partial Y_{l, m}}{\partial \theta}\right)}{\partial \theta}+\frac{1}{\sin ^{2} \theta} \frac{\partial^{2} Y_{l, m}}{\partial \varphi^{2}}+l(l+1) Y_{l, m}=0
$$

cuja a solução são os harmônicos esféricos ${ }^{13}$ (dedução da Eq. 2.22 e Eq. 2.24 no apêndice $\mathrm{E})$.

Aplicando a transformação de variáveis $z=k r$ na equação radial termos:

$$
\frac{\mathrm{d}^{2} R_{n, l}}{\mathrm{~d} z^{2}}+\frac{2}{z} \frac{\mathrm{d} R_{n, l}}{\mathrm{~d} z}+\left(1-\frac{l(l+1)}{z^{2}}\right) R_{n, l}=0,
$$

cujas soluções são combinações de:

$$
\begin{gathered}
j_{l}(z)=(-z)^{l}\left(\frac{\mathrm{d}}{z \mathrm{~d} z}\right)^{l}\left(\frac{\sin z}{z}\right), \\
y_{l}(z)=-(-z)^{l}\left(\frac{\mathrm{d}}{z \mathrm{~d} z}\right)^{l}\left(\frac{\cos z}{z}\right),
\end{gathered}
$$

com $j_{l}(z)$ e $y_{l}(z)$ sendo as funções esférica de Bessel e de Neumann de ordem $l$, respectivamente.

Na Figura 3 é possível ver essas soluções para diferentes valores de $l$. As funções $y_{l}(z)$ divergem para $z=0$, por isso só as funções $j_{l}(z)$ serão utilizadas para a solução da Eq. 2.25. Nossa condição de contorno em $r=a$ implica que $R_{n, l}(a)=0$, portanto os valores de $k$ devem ser escolhidos tais que $z=k a$ corresponda a um dos zeros de $j_{l}(z)$. Denotaremos o n-ésimo zero de $j_{l}(z)$ por $z_{n, l}$. Os níveis de energia possíveis serão ${ }^{13}$

$$
\varepsilon_{n, l}=\frac{\hbar^{2}}{2 m} \frac{z_{n, l}^{2}}{a^{2}}
$$

com degenerescência de $(2 l+1)^{13}$ associada à solução dos harmônicos esféricos. Nosso próximo passo será calcular o cumulante $G(\varepsilon)$ para o caso da esfera, através do cálculo numérico de $z_{n, l}$. 


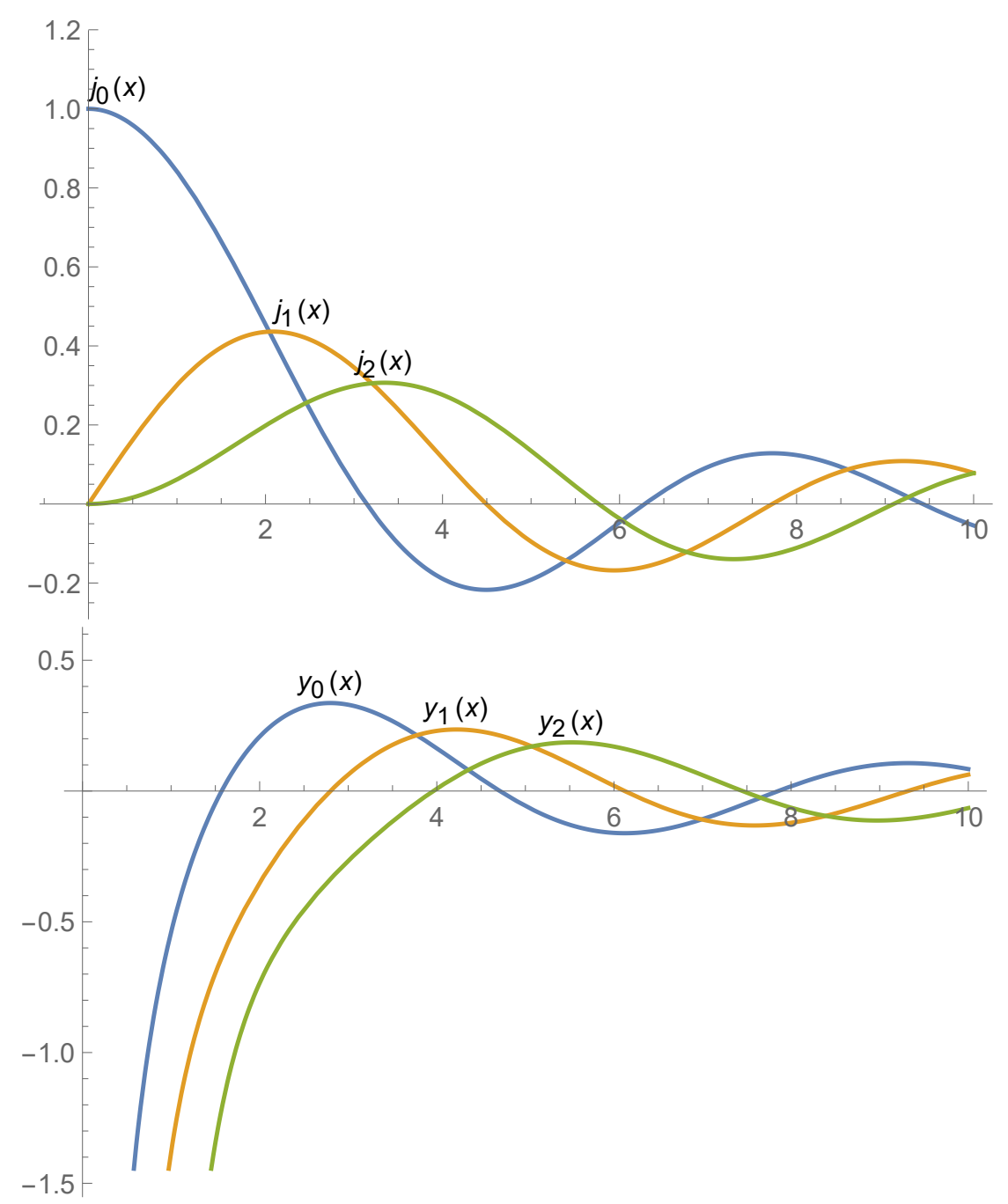

Figura 3: Curvas das três primeiras funções esféricas de Bessel e de Neumann. Fonte: Elaborada pelo autor.

\subsubsection{Método numérico para cálculo do cumulante na caixa esférica}

Nesta subseção iremos calcular o cumulante utilizando métodos numéricos ${ }^{6} \mathrm{e}$ atribuí-lo a uma função analítica que se aproxima do cálculo numérico. O emprego do cumulante é mais eficiente do que da densidade de estados numericamente porque a densidade de estados tem mais flutuações de pontos locais. Por outro lado, o cumulante conta esses pontos reduzindo assim, essas flutuações e agregando menor erro numérico.

Para calcular o cumulante numericamente determinamos todas as raízes até certo valor de energia $\varepsilon$ (obviamente esta terá um único $k$ correspondente) e as contaremos, cada raíz terá peso $(2 l+1)$. O cumulante de $\varepsilon, G(\varepsilon)$ é a dado contagem de todas as raízes (com a degenerescência) até certa energia $\varepsilon$ dada por Eq. 2.28, pois como já argumentado as raízes nos dão os estados de energia possíveis. A Fig. 4 mostra a curva $G(\varepsilon)$ calculada numericamente, até um valor máximo de $\varepsilon=12.000 \varepsilon_{0}$; adotamos que $\varepsilon_{0}=\frac{\hbar^{2}}{2 m a^{2}}$ e $a=10^{-5} \mathrm{~m}$ (valor típico para BEC ${ }^{34}$ ). 
Para encontrarmos as raizes é necessário escrever as funções de Bessel utilizando relações de recorrência, ou seja, para encontrar a l-ésima função basta ter a primeira. Quando a função l-ésima de Bessel inverte o sinal é porque neste intervalo entre o positivo e o negativo há uma raíz. Basta agora montar um contador e acrescentar a degenerescência.

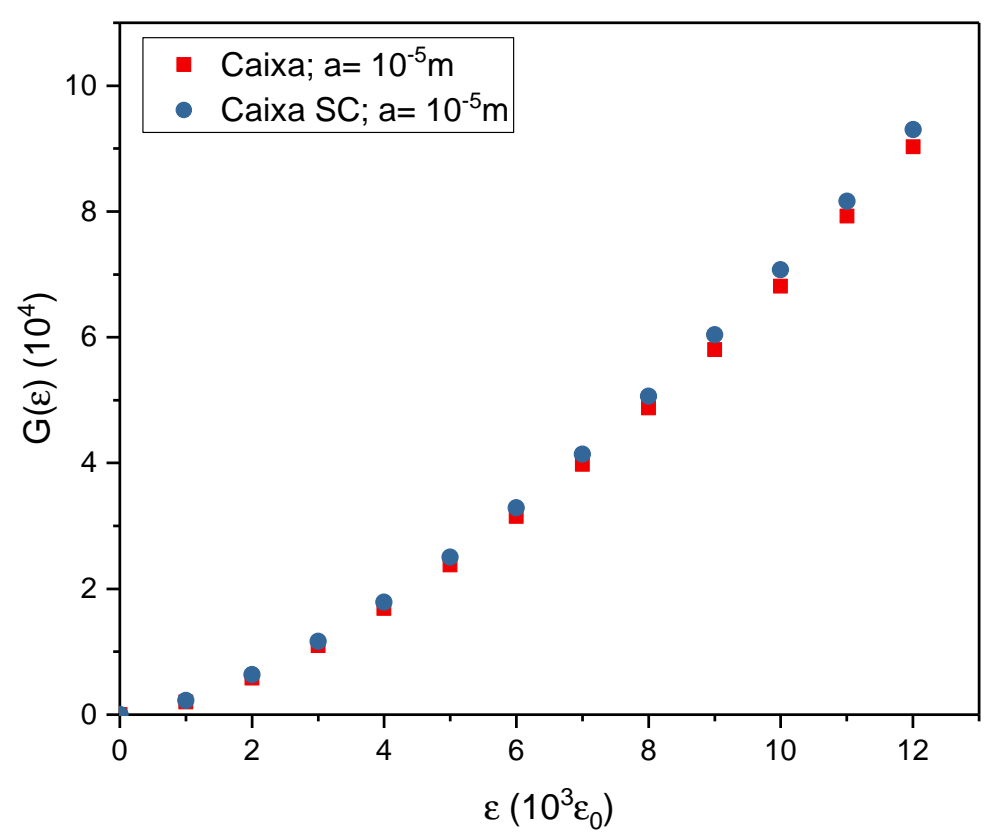

Figura 4: Gráfico do cumulante $G(\varepsilon)$ em função de $\varepsilon$ para $0 \leq \varepsilon \leq 12000 \varepsilon_{0}$, onde $\varepsilon_{0} \frac{\hbar^{2}}{2 m a^{2}}$ e $a=10^{-5} \mathrm{~m}$.

Fonte: Elaborada pelo autor.

De acordo com Fig. 4, notamos que o cumulante obtido através das raízes provenientes da equação de Schrödinger tem valor ligeramente menor do que o cumulante calculado por meio da aproximação semi-clássica, Eq. 2.5.

A Fig. 5nos mostra os valores de $\left(C_{s}-C_{s}\right) / C_{q}$ em função da energia. Podemos ver na figura que quanto maior a energia mais o modelo semi-clássico é eficiente.

Iremos investigar agora a lei de potência de $G(\varepsilon)$. Assumindo que $G(\varepsilon)=C_{\alpha} \varepsilon^{\alpha}$, utilizando a propriedade logarítmica $\ln \left(C_{\alpha} \varepsilon^{\alpha}\right)=\ln \left(C_{\alpha}\right)+\alpha \ln (\varepsilon)$, na Fig. 6 apresentamos $\ln [G(\varepsilon)]$ como função de $\ln (\varepsilon)$ para investigarmos os coeficientes angulares e lineares. O gráfico da Fig. 6 corresponde a Fig. 4 em escala logarítmica. Para cada intervalo entre dois pontos consecutivos da Fig. 6 foi traçada uma reta, o que nos dá 12 coeficientes angulares e lineares de cada intervalo e seus respectivos erros (os erros provem das fluatuações das raizes que não foram representados para melhor visualização do gráfico) estão representados na Fig. 7. Na Fig. 7 também foram apresentados os coeficientes angulares e lineares do modelo semi-clássico. A título de comparação, lembramos que o coeficiente angular do caso tridimensional semi-clássico tem valor $3 / 2$, enquanto que o coeficiente linear vale 


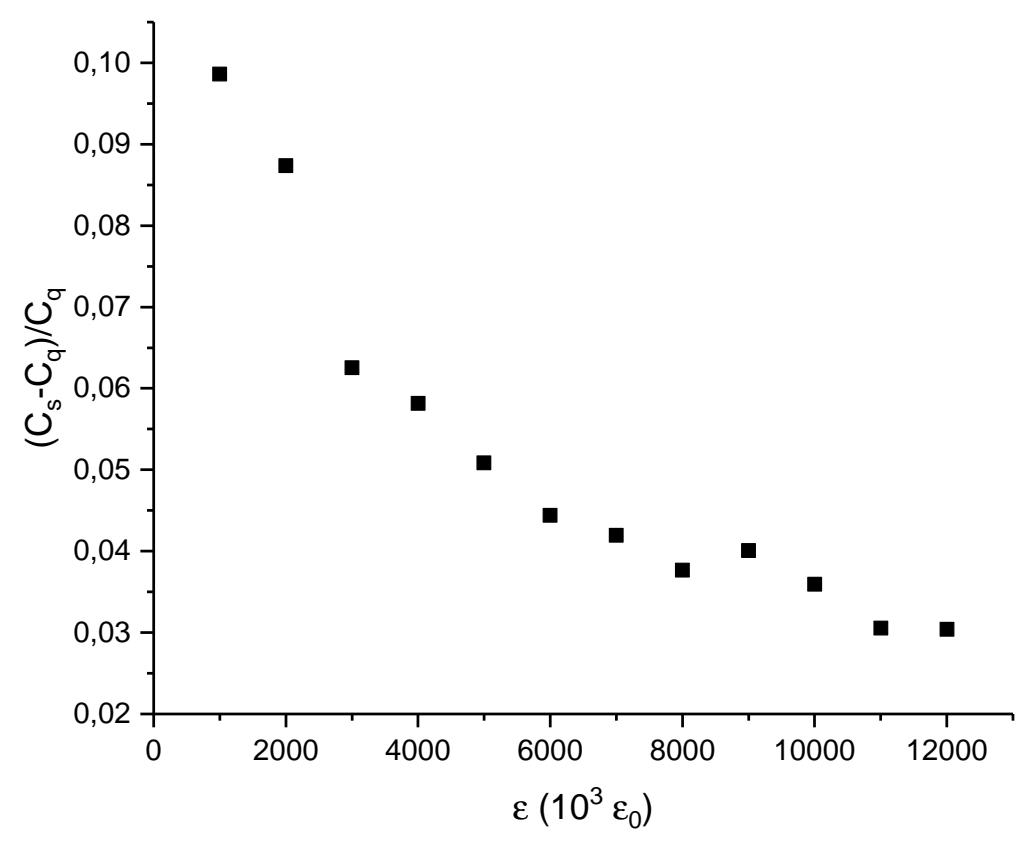

Figura 5: Representação da razão entre a diferença dos valores dos cumulantes da caixa esférica obtidos pelas raizes das equações de Bessel e pela aproximação semiclássica e o próprio cumulante obtido pelas raizes das equações de Bessel.Observe que: $C_{s}$ é o cumulante obtido pela aproximação semi-clássica e $C_{q}$ é o cumulante da caixa obtido pelos autovalores do sistema.

Fonte: Elaborada pelo autor.

$\ln \left(\frac{2}{9 \pi}\right)=-2,64$ (veja Eq. $\left.2.5 \operatorname{com} V=4 \pi a^{3} / 3\right)$

Com os dados da Fig. 7 podemos recuperar o cumulante através de uma função aproximada e analítica em que assumimos que o cumulante $G(\varepsilon)$, sendo $1000 \varepsilon_{0}(i-1) \leq$ $\varepsilon \leq 1000 \varepsilon_{0} i$ e $i$ um número inteiro positivo, será dado por $G(\varepsilon)=C_{\alpha, i}\left[\varepsilon-1000 \varepsilon_{0}(i-1)\right]^{\alpha_{i}}+$ $G\left(1000 \varepsilon_{0}(i-1)\right)$, onde $\ln C_{\alpha, i}$ e $\alpha_{i}$ correspondem ao i-ésimo coeficiente linear e angular respectivamente apresentados na Fig. 7. Utilizaremos essa função analítica para encontrar a densidade de estados e usá-la no cálculo de $T_{c}$ e seu respectivo erro mais adiante nesta Seção. Vale observar que $G(0)=0$.

Observamos que, com o aumento gradativo da energia, o coeficiente angular da reta tende à $3 / 2$ e o coeficiente linear tende a -2,64, ou seja, há uma boa concordância entre os resultados quântico e semi-clássico. A explicação desse limite é dada pelo Teorema de Weyl ${ }^{35}$, que compara as dimensões da caixa com o comprimento de onda da partícula aprisionada. Segundo o Teorema, para o caso em que $\lambda_{d B}^{3} \ll V$, a partícula não seria afetada pelo formato da caixa, e, por isso obtemos o mesmo resultado da aproximação semi-clássica. Para um dado volume da caixa, conforme a energia da partícula cresce, o 


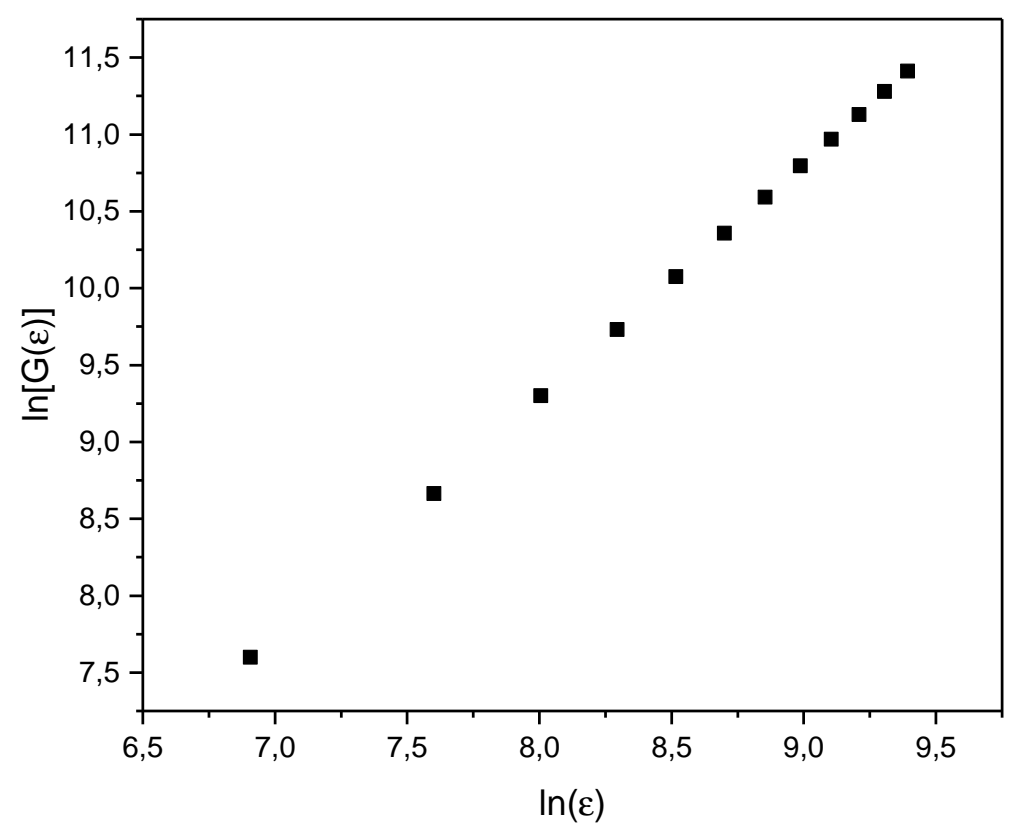

Figura 6: Representação gráfica de $\ln [G(\varepsilon)]$ por $\ln (\varepsilon)$ para $0 \leq \varepsilon \leq 12000 \varepsilon_{0}$, com intervalo de energia de $1000 \varepsilon_{0}$ entre cada ponto.

Fonte: Elaborada pelo autor.

número de onda $k$ aumenta, fazendo com que o valor de $\lambda=2 \pi / k$ diminua, satisfazendo assim cada vez mais a condição de Weyl.

\subsubsection{Cálculo da Temperatura crítica na caixa esférica}

Nesta subseção iremos estimar a temperatura crítica de condensação do BEC na caixa esférica (para um maior aprofundamento ver Ref. ${ }^{36}$ ). Para calcular a $T_{c, q}$ (teperatura crítica através do modelo quântico) do sistema quântico, resolvemos a integral da Eq. 2.15 aplicando $\frac{\mathrm{d} G(\varepsilon)}{\mathrm{d} \varepsilon}$ para a densidade de estados até valores de $\varepsilon=10.000 \varepsilon_{0}$, ou seja, até $i=10$, enquanto que para valores acima de $10.000 \varepsilon_{0}$, utilizamos a expressão da densidade de estados semi-clássica. Além da caixa com raio $a=10^{-5} \mathrm{~m}$, consideramos tabém uma caixa de raio $a=1,33 \times 10^{-5} \mathrm{~m}$, o porquê da escolha deste valor será justificado na seção que segue. As temperaturas críticas são calculadas para $N=10^{5}$. Os dados são apresentados nas Fig. 8 e Fig. 9.

Conforme esperado pela relação entre os cumulantes quântico e semi-clássico (Fig. 4), os valores da $T_{c, q}$ são maiores do que a $T_{c, s}$ (temperatura crítica no modelo semi-clássico), ambos estão mostrados na Fig. 8, embora eles concordem dentro da barra de erro do cálculo numérico. O fato do cumulante semi-clássico ser um pouco maior do que o cumulante do modelo quântico indica que as partículas possuem mais estados de energia disponíveis para uma dada temperatura do sistema, de forma que a saturação da ocupação desses estados, 


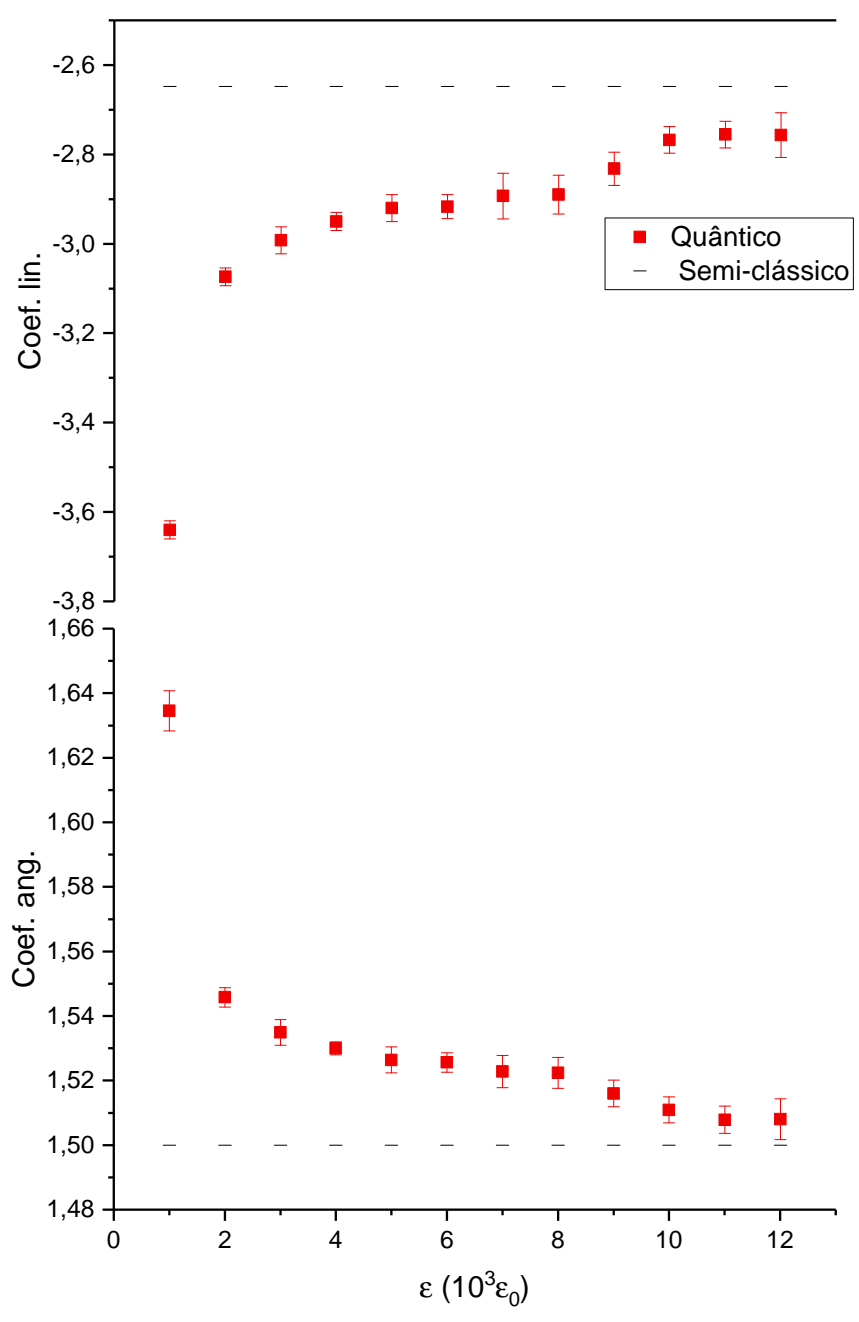

Figura 7: Representação dos coeficientes angulares e lineares (intervalos de energia $10^{3}$ ), vemos que os pontos tendem a linha tracejada (valor dos coeficientes da aproximação semi-clássica) conforme $\varepsilon$ aumenta para diferentes intervalos de momento linear apresentados no quadro.

Fonte: Elaborada pelo autor.

e consequentemente a condensação de Bose-Einstein, deva ocorrer para valores menores da temperatura. Além disso, observamos que a temperatura crítica também diminui com o aumento do raio da caixa mantendo o número de partículas constante, ver Fig. 9.

\subsection{Densidade de Estados em uma Casca Esférica}

Vamos agora considerar uma partícula de massa $m$ aprisionada em um potencial descrito por:

$$
U(r)=\left\{\begin{array}{lc}
0, & a<r<b, \\
\infty & \text { caso contrário. }
\end{array}\right.
$$

O tratamento deste sistema é similar ao caso anterior da caixa esférica. Como a região do potencial não inclui a origem do sistema de coordenados $\mathbf{r}=0$, a parte radial da função 


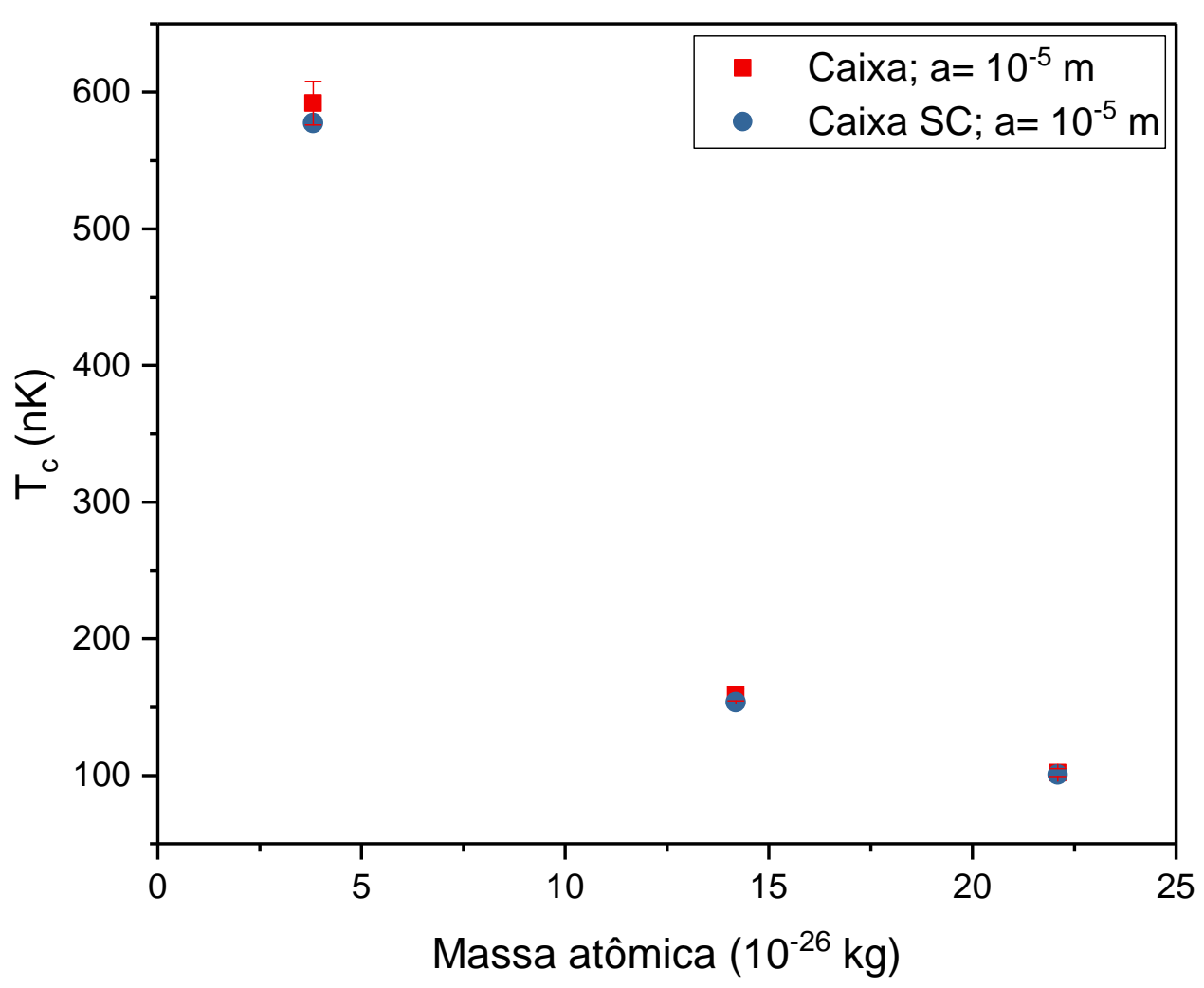

Figura 8: Temperaturas críticas calculadas com os coeficientes obtidos pelo cumulante calculado numericamente para raio $a=10^{-5} \mathrm{~m}$ comparado ao modelo semiclássico.

Fonte: Elaborada pelo autor.

de onda será uma combinação linear das funções esféricas de Bessel e de Neumann ${ }^{37}$ :

$$
R_{n, l}(r)=C j_{l}(k r)+C^{\prime} y_{l}(k r)
$$

onde $C$ e $C^{\prime}$ são constantes a serem definidas pelas condições de contorno e a condição de normalização da função de onda. No entanto, não é de nosso interesse neste texto encontrar tais valores, apenas as autoenergias do sistema.

A condição de contorno impõe que $R_{n, l}$ deve ser zero em $r=a$ e $b$, já que o potencial é infinito nestes pontos, de forma que teremos o sistema de equações:

$$
\begin{aligned}
& R_{n, l}(a)=C j_{l}(k a)+C^{\prime} y_{l}(k a)=0, \\
& R_{n, l}(b)=C j_{l}(k b)+C^{\prime} y_{l}(k b)=0 .
\end{aligned}
$$




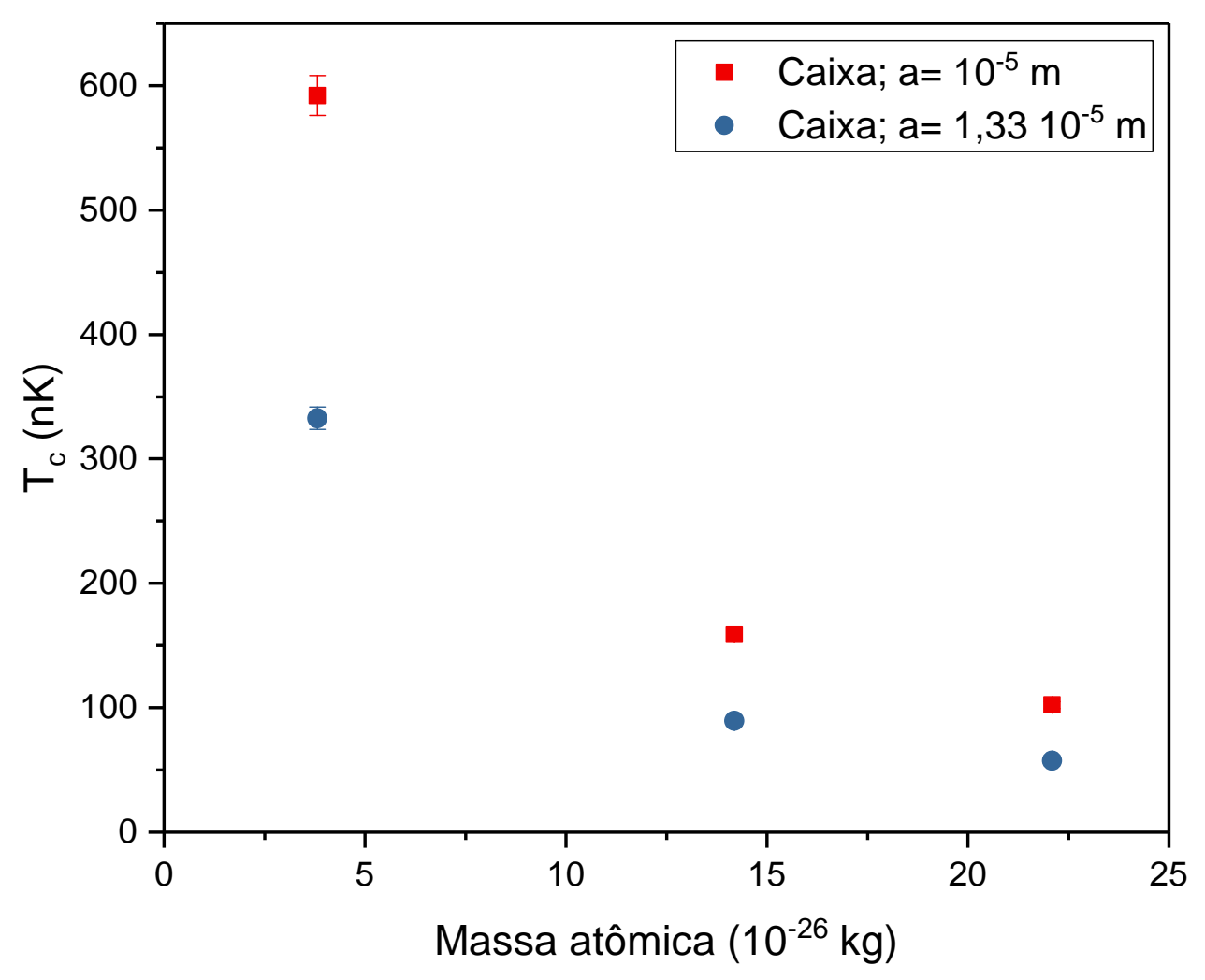

Figura 9: Temperaturas críticas calculadas com os coeficientes obtidos pelo cumulante calculado numericamente para raio $a=1,33 \times 10^{-5} \mathrm{~m}$ comparadas para raio $a=10^{-5} \mathrm{~m}$.

Fonte: Elaborada pelo autor.

Para obter a solução não-trivial desse sistema de equações, o determinante da matriz dos coeficientes do sistema deve ser nulo, o que implica que:

$$
j_{l}(k a) y_{l}(k b)-j_{l}(k b) y_{l}(k a)=0 \text {. }
$$

Uma vez determinadas as raízes da Eq. 2.33, os autoestados serão dados por $\varepsilon=\hbar^{2} k_{n, l}^{2} /(2 m)$ onde $k_{n, l}$ é a n-ésima solução $k$ da l-ésima equação.

\subsubsection{Método numérico para cálculo do cumulante na caixa esférica}

Através dos cálculos numéricos análogos aos feitos na caixa obtivemos o gráfico do cumulante $G(\varepsilon)$ em função de $\varepsilon$, para dois valores do raio interno a $\left(10^{-5} \mathrm{~m} \mathrm{e} 1,5 \times 10^{-5} \mathrm{~m}\right)$, mas com raio externo $b$ fixo $\left(\approx 1,5 \times 10^{-5} \mathrm{~m}\right.$, ordens típicas para BEC $\left.{ }^{34}\right)$, os quais são mostrados na Fig. 10, juntamente com os cumulantes semi-clássicos para estes mesmo raios, mas dessa vez estamos utilizando $\varepsilon_{0}=\frac{\hbar^{2}}{2 m \times\left(10^{-5} \mathrm{~m}\right)^{2}}$. Ainda na Fig. 10 podemos ver 
o cumulante do modelo quântico da caixa de raio igual a $1,33 \times 10^{-5}$. Vale observar que o volume de uma casca de raios interno e externo respectivamente iguais a $10^{-5} \mathrm{~m}$ e $1,5 \times 10^{-5} \mathrm{~m}, V=\frac{4 \pi}{3}\left(1,5^{3}-1^{3}\right) 10^{-15} \mathrm{~m}^{3}=9,94 \times 10^{-15} \mathrm{~m}^{3}$, é o mesmo que o de uma caixa esférica de raio $1,33 \times 10^{-5} \mathrm{~m}^{3}, V=\frac{4 \pi}{3}(1,33)^{3} \times 10^{-15} \mathrm{~m}^{3}=9,94 \times 10^{-15} \mathrm{~m}^{3}$. Então na Fig. 10 vemos que o cumulante do modelo quântico da caixa é maior do que o da casca de mesmo volume, apesar de ser pequena a diferença. Ainda na mesma figura vemos que o cumulante de uma casca de volume maior (raio interno menor) tem cumulante maior do que a de volume menor ainda mantendo o número de partículas. Também mostrado na Fig. 10, igualmente ao caso da caixa esférica, na casca esférica o cumulante do modelo quântico é menor do que o correspondente semi-clássico.

A fim de explorar melhor o sistema da casca esférica, investigamos a lei de potência

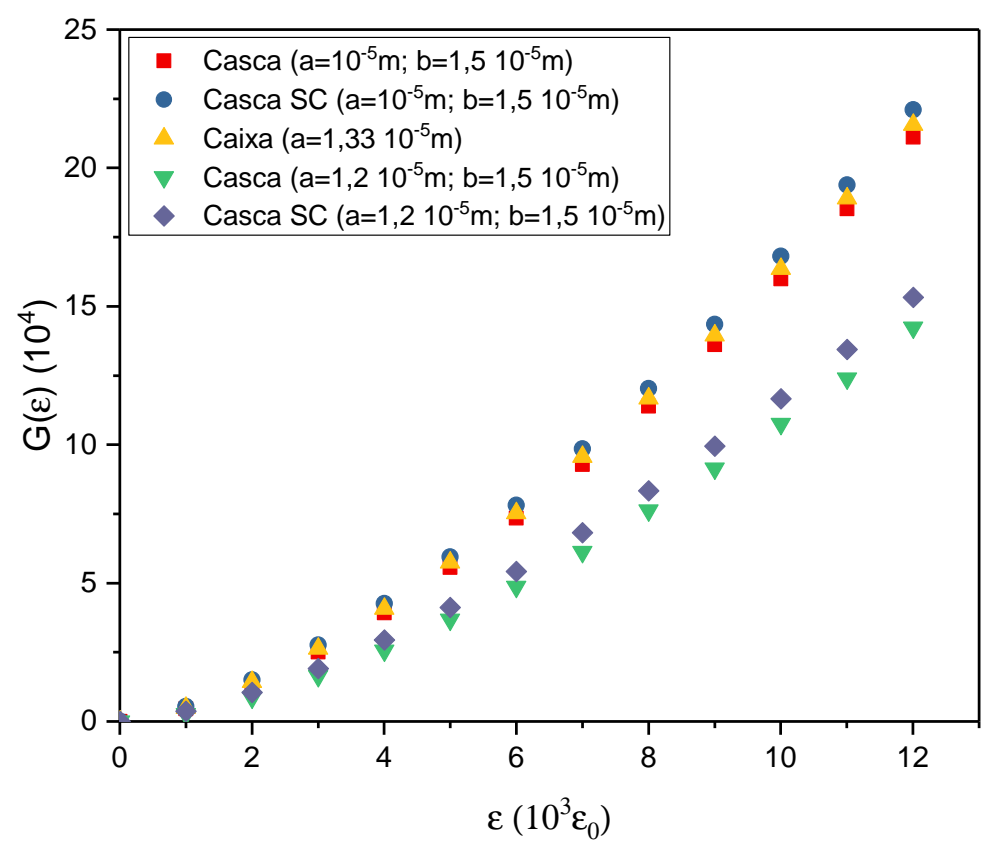

Figura 10: Cumulantes da caixa e da casca esféricas no regime quântico e semi-clássico. Fonte: Elaborada pelo autor.

de $G(\varepsilon)$. Na Fig. 11 mostramos o gráfico com os coeficientes angulares e lineares resultantes da aplicação do logaritmo em ambos os eixos do gráfico de $G(\varepsilon)$ por $\varepsilon$, para diferentes espessuras da casca $\delta R=b-a$ mantendo $b=1,5 \times 10^{-5} \mathrm{~m}$ fixo e variando $a$ e diferentes valores de $k$, ou seja, diferentes valores de $\varepsilon$. Como argumentado na Seção anterior, quanto maior $\varepsilon$ e,consequentemente maior o $k$, mais o regime quântico se aproxima do regime semi-clássico. Considerando o volume da casca esférica $V=4 \pi\left(b^{3}-a^{3}\right) / 3=$ $4 \pi\left[(a+\delta R)^{3}-a^{3}\right] / 3$, o cumulante dado pela aproximação semi-clássica terá coeficiente linear dado por $\ln \left\{2\left[(a+\delta R)^{3}-a^{3}\right] /(9 \pi)\right\}$. Para maiores valores de $k$ (implica em menor $\left.\lambda_{d B}\right)$ e de $\delta R$ (implica em maior volume), mais o sistema se aproxima do regime semi- 
clássico, consistente com a condição de Weyl.

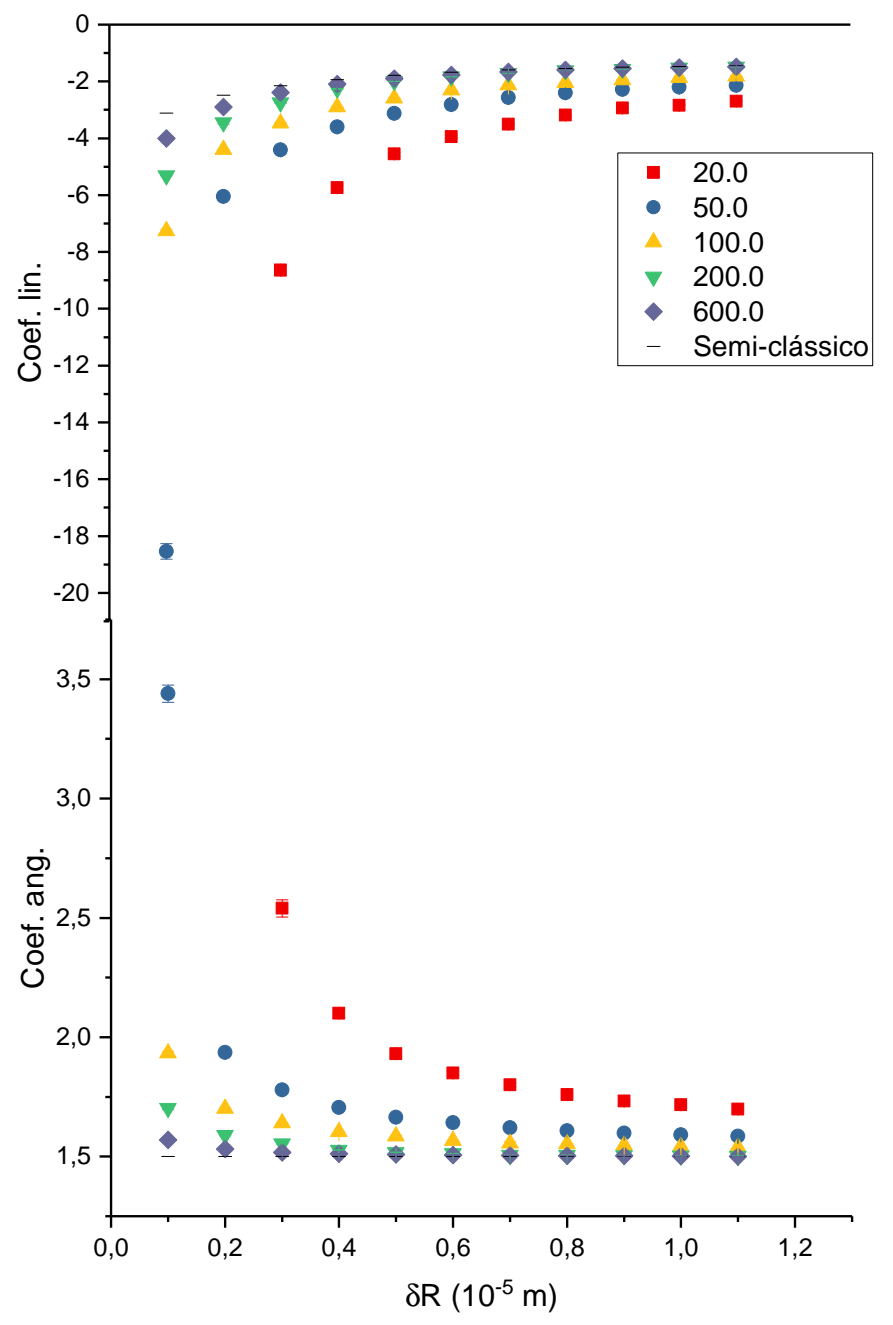

Figura 11: Representação dos coeficientes angulares e lineares das funções $\ln [G(\varepsilon)]$ por $\ln (\varepsilon)$ para diferentes raio internos $a$, onde $\delta R=(b-a)$. Fonte: Elaborada pelo autor.

\subsubsection{Cálculo da temperatura crítica na casca esférica}

Nesta subseção calcularemos as temperaturas críticas numericamente e pelo modelo semi-clássico (para um maior aprofundamento ver Ref. ${ }^{36}$ ). Para isso utilizaremos $a=10^{-5} \mathrm{~m}$ e $b=1,5 \times 10^{-5} \mathrm{~m}$ e calcularemos a $T_{c}$ do mesmo modo que na Seção anterior. Também calcularemos o valor da $T_{c, q 2}$, que corresponde à temperatura crítica para $a=1,2 \times 10^{-5} \mathrm{~m}$ e $b=1,5 \times 10^{-5} \mathrm{~m}$. Os valores podem ser observados na Fig. 12 e na Fig. 13 , novamente para o número total de átomos $N=10^{5}$.

Comparando as temperaturas críticas da casca e caixa esférica de mesmo volume (ver Fig. 13), vemos que a primeira apresenta valores maiores de $T_{c}$. De acordo com 


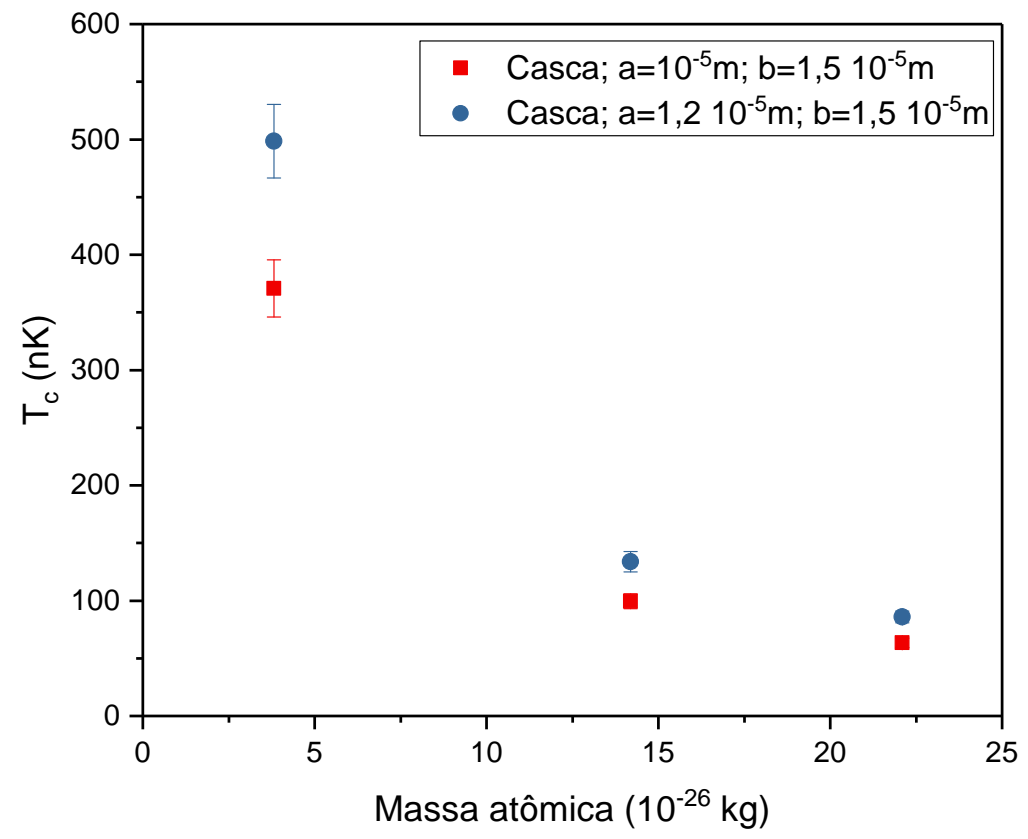

Figura 12: Temperaturas críticas para diferentes raios que formam a casca esférica. Fonte: Elaborada pelo autor.

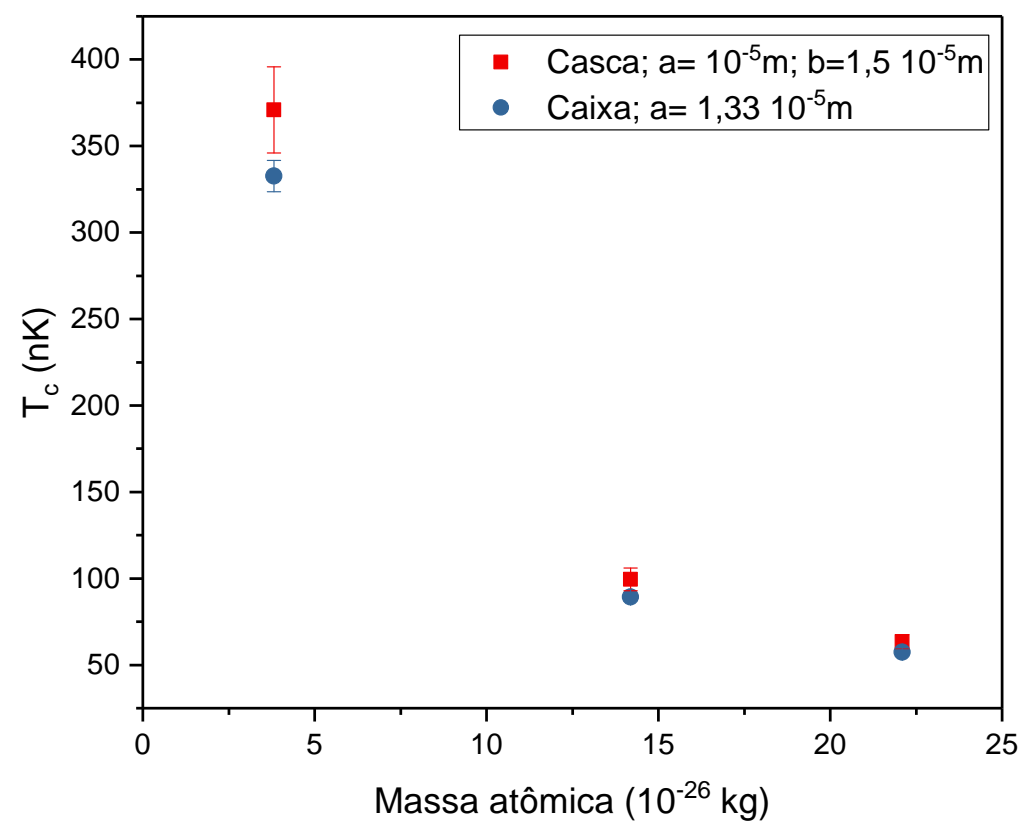

Figura 13: Comparação de temperaturas críticas de mesmo volume mas diferentes formatos (caixa e casca).

Fonte: Elaborada pelo autor. 
Fig. 10, o cumulante da casca esférica é menor do que o da caixa esférica para dada energia, justificando a relação entre as temperaturas. Outra observação relevante é a de que conforme o raio interno se aproxima do raio externo a temperatura crítica para um mesmo número de partículas aumenta conforme pode-se ver na Fig. 12.

\subsubsection{Casca puramente 2D}

Nos resta agora investigar o caso no limite onde o raio interno tende ao raio externo, fazendo com que a casca fique cada vez mais fina. Para isso vamos considerar que $b=a+\Delta$. A Eq. 2.33 fica da forma:

$$
j_{l}(k a) y_{l}[k(a+\Delta)]-j_{l}[k(a+\Delta)] y_{l}(k a)=0 .
$$

A expansão de Taylor para $\Delta=0$ resulta em:

$$
j_{l}[k(a+\Delta)]=j_{l}(k a)+\left.\frac{\mathrm{d} j_{l}[k(a+\Delta)]}{\mathrm{d} \Delta}\right|_{\Delta=0} \Delta+\mathscr{O}\left(\Delta^{2}\right) .
$$

A derivada da Eq. 2.35 é dada pela propriedade das funções esféricas de Bessel e de Neumann ${ }^{37}$ :

$$
\frac{\mathrm{d} j_{l}[k(a+\Delta)]}{\mathrm{d}(a+\Delta)}=\left\{\frac{l}{a+\Delta} j_{l}[k(a+\Delta)]-j_{l+1}[k(a)]\right\} .
$$

O mesmo procedimento adotado nas Eq. 2.35 e Eq. 2.36 se aplica a $y_{l}[k(a+\Delta)]$. Substituindo essas relações na Eq. 2.34, o mesmo para as funções de Neumann, e assumindo que $\Delta \rightarrow 0$ (desprezando termo de ordem $\mathscr{O}\left(\Delta^{2}\right)$ ou superior) teremos, após alguma álgebra:

$$
-y_{l}(k a) j_{l+1}(k a)+j_{l}(k a) y_{l+1}(k a)=0 .
$$

Considerando a seguinte propriedade das funções esféricas de Bessel e de Neumann nos dá 37 :

$$
-y_{l}(k a) j_{l+1}(k a)+j_{l}(k a) y_{l+1}(k a)=\frac{1}{(k a)^{2}} .
$$

Temos que comparando as Eq. 2.37 e Eq. 2.38, concluímos que:

$$
\frac{1}{k a}=0
$$

A Eq. 2.39 mostra que as raízes que satisfazem a Eq. 2.34 tendem a um mesmo valor $k \rightarrow \infty$, para um dado valor fixo do raio $a$, ou seja, quando o raio interno está muito próximo do raio externo da casca temos uma solução incoerente para se determinar o cumulante. Essa incoerência também é percebida nos cálculos numéricos porque as raízes das funções esféricas de Bessel e de Neumann admitem valores muito grandes e suas flutuações tornam o método utilizado para calcular a $T_{c}$ inútil por causa do elevado erro das retas traçadas. A questão aqui seria que os espaçamentos entre os níveis de energia em r são muito grandes frente as escalas de energia relevantes do sistema, de tal forma que 
extingue a possibilidade de excitar uma partícula para os níveis excitados de r. A solução para esse impasse seria desconsiderar os graus de liberdade radiais do sistema.

Se pensarmos no confinamento puramente $2 D$ da partícula, essa terá apenas graus de liberdade angulares e nenhum radial. Dessa forma, para encontrar os estados possíveis desse sistema vamos utilizar a equação de Schrödinger simplificada, ou seja, sem a parte radial:

$$
\frac{-\hbar^{2}}{2 m} \nabla^{2} Y_{l, m}=\frac{\hbar^{2}}{2 m} \frac{l(l+1)}{a^{2}} Y_{l, m}=E_{l} Y_{l, n}
$$

onde $a$ é o raio e $l$ é um número inteiro positivo. Assim extraimos trivialmente que:

$$
E_{l}=\frac{\hbar^{2}}{2 m} \frac{l(l+1)}{a^{2}}=l(l+1) E_{0}
$$

onde $E_{0}=\frac{\hbar^{2}}{2 m a^{2}}$. Como já argumentado antes, a degenerescência dos níveis de energia será $2 l+1$. Podemos reescrever a Eq. 2.41 da seguinte forma:

$$
E_{l}=E_{0}(l+1 / 2)^{2}-\frac{E_{0}}{4}
$$

e definir $E^{\prime}$ :

$$
E_{l}+\frac{E_{0}}{4}=E^{\prime}=(l+1 / 2)^{2} E_{0}
$$

A Eq. 2.42 é muito similar a Eq. 2.6 então a densidade de estados sem degenerescência será $g\left(E^{\prime}\right) d E^{\prime}=\frac{1}{2 \sqrt{E^{\prime} E_{0}}} d E^{\prime}$. Adicionando a degenerescência, porém, teremos $g\left(E^{\prime}\right) d E^{\prime}=(2 l+1 / 2) \frac{1}{\sqrt{E^{\prime} E_{0}}} d E^{\prime}$. Substituindo agora $E^{\prime}$ por $l$, teremos $g\left(E^{\prime}\right) d E^{\prime}=(2 l+1) d l$. Agora que já temos a densidade de estados, para calcular $T_{c}$ basta usar a integral da Eq. 2.15, mas com uma pequena diferença: a integração será em $l$. Como queremos contabilizar as partículas excitadas, iremos empregar o limite de $l=1$ até $\infty^{38}$.

$$
N=\int_{2 E_{0}}^{\infty} g\left(E^{\prime}\right) N_{B}\left(E^{\prime}\right) d E^{\prime}=\int_{1}^{\infty} \frac{(2 l+1)}{e^{-E_{0} l(l+1) / k_{B} T_{c}}-1} d l .
$$

Expressando a função de Bose na forma de somatório, da Eq. 2.44 teremos:

$$
N=\sum_{n=1}^{\infty} \int_{1}^{\infty}(2 l+1) e^{-n E_{0} / k_{B} T_{c} l(l+1)} d l=\sum_{n=1}^{\infty} \frac{e^{-2 n E_{0} / k_{B} T_{c}}}{n E_{0} / k_{B} T_{c}} .
$$

Resolvendo o somatório:

$$
N=2-\frac{\ln \left(e^{2 E_{0} / k_{B} T_{c}}-1\right)}{E_{0} / k_{B} T_{c}}
$$

Recuperando o valor de $E_{0}$ :

$$
N=2-\frac{2 m a^{2} k_{B} T_{c}}{\hbar^{2}} \ln \left(e^{\frac{\hbar^{2}}{m a^{2} k_{B} T_{c}}}-1\right) .
$$

Considerando os valores $N=10^{5}, a=1,5 \times 10^{-5} \mathrm{~m}$ e $m=1,419 \times 10^{-25} \mathrm{~kg}\left({ }^{87} \mathrm{Rb}\right)$ na Eq. 2.47, teremos $T_{c} \approx 145,3 \mathrm{nK}$. Este resultado é coerente com a casca esférica $3 \mathrm{D}$, visto que quando o raio interno aumenta de $1,0 \times 10^{-5} \mathrm{~m}$ para $1,2 \times 10^{-5} \mathrm{~m}$, a temperatura 
crítica também tende a um valor maior (varia de 99, $54 \mathrm{nK}$ para 135,0 nK). Perceba que na Eq. 2.47 o termo $a^{2}$ sempre aparece multiplicando $T_{c}$ e vice-versa, ou seja, a $T_{c}$ é inversamente proporcional a $a^{2}$. Concluimos que para o caso do raio interno tender ao infinito, a temperatura crítica tenderá a zero, o que seria o mesmo que dizer que a $T_{c}$ é zero no plano porque se $a \rightarrow \infty$ a casca puramente $2 \mathrm{D}$ vira um plano.

Vamos agora discutir a casca esférica no caso semi-clássico. A densidade de estados na aproximação semi-clássica para potencial nulo $g_{2 D}(\varepsilon)$ é dada por (ver apêndice $\mathrm{F}$ ):

$$
g_{2 D}(\varepsilon)=\frac{m}{2 \pi} \frac{A}{\hbar^{2}}
$$

onde $A$ é a área onde a partícula está confinada. Perceba que se $g_{2 D}(\varepsilon)$ for escrita na forma $g_{2 D}(\varepsilon)=C_{\alpha} \varepsilon^{\alpha-1}$ temos que $\alpha=1$. Se utilizarmos a Eq. 2.16 para calcularmos a $T_{c}$ temos que $T_{c}=0$, pois quando $\alpha=1, \zeta(\alpha) \rightarrow \infty$. O que justifica a discrepância entre o resulado semi-clássico e o quântico é o Teorema de Weyl. Para o caso em que $A / \lambda_{d B}^{2} \gg 1$, ou seja, para o sistema quântico tender ao semi-clássico, a condição de Weyl só será satisfeita se $a \rightarrow \infty$. Conforme discutido anteriormente, neste limite temos que $T_{c} \rightarrow 0$ no regime quântico, com a convergência ao regime de validade do cálculo semi-clássico. 



\section{EQUAÇÃO DE GROSS-PITAEVSKII}

Neste Capítulo iremos desenvolver a teoria para gases não uniformes diluídos.

Trata-se de um tópico de estudo importante pelo fato de que experimentalmente o condensado de Bose-Einstein em gases atômicos armadilhados é um sistema não uniforme.

\subsection{Introdução da Equação de Gross-Pitaevskii.}

O gás de Bose ideal considerado no Capítulo anterior é um sistema muito peculiar. Quando consideramos a interação entre as partículas, as propriedades desse gás serão afetadas de forma dramática, mesmo para amostras bem diluídas desse gás. Mesmo o problema do gás fracamente interagente não é trivial, já que o estado fundamental tem energia zero na ausência de interação, sendo a teoria de perturbação incapaz de trazer resultados satisfatórios ${ }^{1}$.

Considere que todas as partículas de um gás diluído de bósons condensado estão no estado fundamental, ou seja, $N_{0}=N$.

Para investigar a energia de muitos corpos ${ }^{1,39}$ vamos assumir que a função de onda é o produto simetrizado de cada função de partícula única. Em um BEC puro todos os bósons estão no mesmo estado de energia, $\phi(\mathbf{r})$, então podemos escrever a função de onda de um sistema de $N$ partículas como

$$
\Phi\left(\mathbf{r}_{1}, \mathbf{r}_{2} \ldots, \mathbf{r}_{N}\right)=\phi\left(\mathbf{r}_{i}\right)
$$

É conveniente introduzir o conceito de função de onda $\Psi(\mathbf{r})$ de um condensado pela definição

$$
\Psi(\mathbf{r})=N^{1 / 2} \phi(\mathbf{r})
$$

A densidade $n(\mathbf{r})$ é dada por:

$$
n(\mathbf{r})=|\Psi(\mathbf{r})|^{2}
$$

Consequentemente o número de partículas $N$ pode ser calculado da forma:

$$
N=\int d V n(\mathbf{r})=\int d V|\Psi(\mathbf{r})|^{2} .
$$

Pelo fato de considerarmos os gases diluídos temos que, $r_{0} \ll d$, onde $r_{0}$ representa o alcance das forças interatômicas e $d$ a distância média entre os átomos $d=n^{-1 / 3}$, sendo $n=N / V$ a densidade do gás. Esta condição permite que consideremos apenas a interação entre pares de partículas, com a possibilidade de negligenciarmos a colisão de três ou mais partículas.

A distância entre as partícula é sempre grande o suficiente para justificar a expressão 
assintótica para a função de onda do movimento relativo entre as partículas, a qual fixa a amplitude de espalhamento. Como consideramos baixos valores de energia, a amplitude de espalhamento se torna independente da energia e do ângulo de espalhamento. Teremos a chamada seção de choque de onda s $\left(a_{s}\right)$, a qual caracteriza todos os efeitos da interação nas propriedades físicas do gás. Em geral, consideramos o gás como fracamente interagente se a condição $\left|a_{s}\right| \ll d$ for satisfeita. Essa condição é equivalente a considerarmos um valor pequeno do parâmetro $n\left|a_{s}\right|^{3}$.

Então nesta aproximação o potencial de interação tem característica de uma função delta de Dirac. As constantes que multiplicam essa delta são $4 \pi \hbar^{2} a_{s} / m$, onde $a_{s}$ corresponde a seção de choque atômica (se for negativo a interação é atrativa, caso contrário, repulsiva). Portanto tem-se que o potencial de interação $g$ é dada por ${ }^{27}$ :

$$
g=\frac{4 \pi \hbar^{2} a_{s}}{m} \delta\left(\mathbf{r}-\mathbf{r}^{\prime}\right)=g_{0} \delta\left(\mathbf{r}-\mathbf{r}^{\prime}\right)
$$

onde $g_{0}$ é a constante que multiplica a delta, $\mathbf{r}$ é a posição de uma partícula e $\mathbf{r}^{\prime}$ a posição da outra.

A Hamiltoniana $H$ do sistema pode ser escrita em função dos momentos $\mathbf{p}_{i}$ (momento da partícula $i$ ) e do potencial externo $V\left(\mathbf{r}_{i}\right)$ que atua na partícula de posição $\mathbf{r}_{i}$, e será dada por:

$$
H=\sum_{i=1}^{N}\left\{\left[\frac{\mathbf{p}_{i}^{2}}{2 m}+V\left(\mathbf{r}_{i}\right)\right]+g_{0} \sum_{i<j} \delta\left(\mathbf{r}_{i}-\mathbf{r}_{j}\right)\right\} .
$$

Para $N$ muito grande a energia do sistema proveniente da Hamiltoniana será ${ }^{39}$ :

$$
E_{s i s}=\int d V\left[\frac{\hbar^{2}}{2 m}|\nabla \Psi(\mathbf{r})|+V(\mathbf{r})|\Psi(\mathbf{r})|^{2}+\frac{1}{2} g_{0}|\Psi(\mathbf{r})|^{4}\right] .
$$

O primeiro termo da integral corresponde à energia cinética, o segundo termo corresponde à energia potencial da partícula devido ao campo externo e o último termo já discutido é a energia de interação. Observe que a densidade de energia de interação depende da função de onda elevada à quarta potência, o que faz todo o sentido porque duas moléculas devem estar no mesmo $\mathbf{r}$ para que haja interação: a probabilidade de uma estar em $\mathbf{r}$ é $|\Psi(\mathbf{r})|^{2}$ e ambas em $\mathbf{r}$ é $\left(|\Psi(\mathbf{r})|^{2}\right)^{2}=|\Psi(\mathbf{r})|^{4}$. Se aplicarmos a Eq. 3.3 na energia de interação vê-se que a ela depende da integral do quadrado da densidade $\int n^{2} d V$, ou seja, a energia de interação aumenta com a densidade.

Agora vamos analisar o raio médio $R$ da nuvem, que minimiza a energia por partícula $\varepsilon_{p}$. No entanto, vamos inicialmente encontrar o parâmetro $d_{0}$ que equaliza a ordem da energia cinética e da potencial. A ordem da energia cinética de uma partícula é 
$\sim \hbar^{2} / 2 m R^{2}$, a da energia potencial harmônica é $\sim m w_{0}^{2} R^{2} / 2$. Logo ambas são da mesma ordem se a nuvem for do tamanho $\left(d_{0}\right)$ :

$$
d_{0}=\sqrt{\frac{\hbar}{m w_{0}}},
$$

onde $w_{0}=\left(w_{x} w_{y} w_{z}\right)^{\frac{1}{3}}$.

A energia de interação por partícula é $\sim n g_{0}=3 N \hbar^{2} a_{s} / R^{3}$. Então a energia de uma partícula $\varepsilon_{p}$ será $\sim \hbar^{2} /\left(2 m R^{2}\right)+m w_{0}^{2} R^{2} / 2+3 N \hbar^{2} a_{s} / R^{3}$. Para encontrar o $R$ que minimiza a magnitude de $\varepsilon_{p}$ vamos derivar essa expressão com respeito a $R$ e igualar a zero, isto é:

$$
\frac{\mathrm{d} \varepsilon_{p}}{\mathrm{~d} R} \sim-\frac{\hbar^{2}}{m R^{3}}+m w_{0}^{2} R-\frac{9 N \hbar^{2} a_{s}}{R^{4}} \sim 0
$$

Pela transformação de variável $R=d_{0} y$ e pela multiplicação de $\frac{m}{d_{0} \hbar^{2}}$ em ambos lados da igualdade na Eq. 3.9, temos

$$
-y+y^{5}-\frac{9 N a_{s}}{d_{0}} \sim 0
$$

Considerando as ordens de grandezas típicas: $N \sim 10^{6}, a_{s} \sim 10^{-9} \mathrm{~m}$ e $d_{0} \sim 10^{-6} \mathrm{~m}$ é possível concluir que a grandeza adimensional $\frac{N a_{s}}{d_{0}} \gg 1$. Isso significa que para resolver a Eq. 3.10 y deverá ser um número muito grande, ou seja, $y^{5} \gg y$, portanto o termo linear pouco irá contriuir para solução. Trivialmente obtemos o valor de $y$,

$$
y \approx\left(\frac{N a_{s}}{d_{0}}\right)^{1 / 5}
$$

e a magnitude de $R$

$$
R \sim d_{0}\left(\frac{N a_{s}}{d_{0}}\right)^{1 / 5}
$$

Se aplicarmos o valor da magnitude de $R$ na $\varepsilon_{p}$ podemos concluir que a ordem de grandeza da energia do sistema $E_{s i s}=\varepsilon_{p} N$ será

$$
E_{s i s} \sim N \hbar w_{0}\left(\frac{N a_{s}}{d_{0}}\right)^{\frac{2}{5}}
$$

Também nos será conveniente mais adiante no texto comparar a magnitude da energia cinética e de interação para definirmos uma escala de comprimento $\xi$ :

$$
\xi=\frac{\hbar}{\sqrt{8 \pi a_{s} n}}
$$

Se aplicarmos o valor da magnitude de $R$ na energias cinética, potencial e de interação obteremos $\sim \hbar w_{0} /\left(\frac{N a_{s}}{d_{0}}\right)^{\frac{2}{5}}, \sim \hbar w_{0}\left(\frac{N a_{s}}{d_{0}}\right)^{\frac{2}{5}}$ e $\sim \hbar w_{0}\left(\frac{N a_{s}}{d_{0}}\right)^{\frac{2}{5}}$, respectivamente. 
Assim sendo, podemos concluir que a energia potencial e de interação contribuirão muito mais para a energia do sistema do que a parte cinética. Adiante iremos utilizar uma aproximação onde desprezamos o termo da energia cinética para solucionar a Equação de Gross-Pitaevskii, a qual provem da minimização da energia.

A minimização de $E_{\text {sis }}\left(\delta E_{\text {sis }}-\mu N=0\right)$ da Eq. 3.7 com respeito à $\Psi^{*}$ resulta na equação de Gross-Pitaevskii independente do tempo (GPE) ${ }^{1}$ :

$$
\left(-\frac{\hbar^{2}}{2 m} \nabla^{2}+V(\mathbf{r})+g_{0}|\Psi(\mathbf{r})|^{2}\right) \Psi(\mathbf{r})=\mu \Psi(\mathbf{r}) .
$$

Observe que mantivemos o $\mu$ como multiplicador de Lagrange e mantivemos o número de partículas $N$ conservado. A minimização da energia é a que nos dá a função de onda porque todo sistema físico tende ao minimo de energia. Essa equação foi derivada independentemente por Gross (1961) e Pitaevskii (1961) ${ }^{27,40}$ e consiste na ferramenta teórica principal para investigar gases de Bose diluídos a baixas temperaturas. Trata-se de uma equação de campo médio com parâmetro de ordem $\Psi$ sendo determinado de forma auto-consistente.

Vale ressaltar que essa equação para o parâmetro de ordem $\Psi$ tem o papel análogo às equações de Maxwell na eletrodinâmica clássica. Conforme ressaltado anteriormente, a função de onda do condensado representa o limite clássico da onda de de Broglie, onde os aspectos corpusculares da matéria não são mais relevantes. Diferentemente das equações de Maxwell, porém, a GPE contém a constante quântica de Planck explicitamente. Isso atrela os fenômenos de coerência, como interferência, ao valor dessa constante. Outra peculiaridade da GPE advém do seu termo não-linear. Este se origina da interação entre as partículas e introduz uma importante analogia entre a condensação de Bose-Einstein em gases atômicos e ótica não-linear. Efeitos de interação e coerência são importantes características que podem ser investigadas a partir da equação de Gross-Pitaevskii e que tornam a física dos condensados de Bose-Einstein um campo de estudo muito rico para pesquisa teórica e experimental ${ }^{1}$.

Como a energia cinética pouco contribui para a Eq. 3.7, podemos desprezar o primeiro termo na Eq. 3.15 e considerar que a nuvem terá um tamanho da ordem de $R$ dado em Eq. 3.12. Essa é a chamada aproximação de Thomas-Fermi (TF). Nesse regime de TF as Eqs. 3.7 e 3.15 podem ser escritas na forma:

$$
\begin{gathered}
E_{\text {sis }} \approx \int d V\left[V(\mathbf{r})|\Psi(\mathbf{r})|^{2}+\frac{1}{2} g_{0}|\Psi(\mathbf{r})|^{4}\right] \\
\left(V(\mathbf{r})+g_{0}|\Psi(\mathbf{r})|^{2}\right) \Psi(\mathbf{r})=\mu \Psi(\mathbf{r})
\end{gathered}
$$


A Eq. 3.17 pode ser resolvida algebricamente para $|\Psi|^{2}$, fornecendo o perfil para a densidade atômica:

$$
n(\mathbf{r})=\frac{\mu-V(\mathbf{r})}{g_{0}} .
$$

Podemos considerar o ponto onde a densidade vai a zero $n\left(R_{0}\right)=0$, e definir o potencial químico em termos de $R_{0}$ para potencial harmônico $V(\mathbf{r})$ dado pela Eq. 2.11:

$$
\mu=V\left(\mathbf{R}_{0}\right)=\frac{m}{2} w_{0}^{2} R_{0}^{2}
$$

onde $R_{0}^{3}=R_{x} R_{y} R_{z}$. Se substituirmos o valor do potencial químico na Eq. 3.18 obtemos:

$$
n(\mathbf{r})=n(0)\left(1-\frac{R^{2}}{R_{0}^{2}}\right),
$$

onde $n(0)=\mu / g_{0}=R_{0}^{2} /\left(8 \pi a_{s} d_{0}^{4}\right)$ e $R_{i}$ corresponde aos raios máximos nas coordenadas cartesianas $\left(R_{x}, R_{y}\right.$ e $\left.R_{z}\right)$. Pela Eq. 3.20 é possível ver que a nuvem do condensado terá o formato de um elipsóide de secção parabólica.

Ao aplicar o resultado encontrado na Eq. 3.20 em Eq. 3.4 (condição de normalização) é possível encontrar o raio $R_{0}$ da nuvem em termos do número de partículas condensadas:

$$
N=\frac{8 \pi n(0) R_{0}^{3}}{15}
$$

Ao substituir o valor de $n(0)$ na Eq.3.21 temos que:

$$
\frac{R_{0}^{5}}{d_{0}^{5}}=15 \frac{N a_{s}}{d_{0}} .
$$

Utilizando a Eq. 3.22 é possível escrever a Eq. 3.19 somente em termos de $\hbar, w_{0}$ e $d_{0}$ :

$$
\mu=\frac{\hbar w_{0}}{2}\left(\frac{15 N a_{s}}{d_{0}}\right)^{\frac{2}{5}} .
$$

Finalmente, a energia $E_{\text {sis }}$ pode ser encontrada através relação termodinâmica $\mu=\frac{\partial E_{s i s}}{\partial N}$, resultando na equação:

$$
E_{\text {sis }}=\frac{5}{7} \mu N
$$

Para comparar as variáveis $\xi, d_{0}$ e $R_{0}$, consideramos o o pico de densidade da nuvem $n(0)$ dado na Eq. 3.18. De acordo com a Eq. 3.22 obtemos que

$$
\xi=\frac{1}{\sqrt{\pi a_{s} n(0)}}=\frac{d_{0}^{2}}{R_{0}} .
$$

Sabendo que $R_{0} \gg d_{0}$, concluimos que $\xi \ll d_{0}$. Portanto, a energia de interação é muito maior do que a cinética. 


\subsection{Equação de Gross-Pitaevskii dependente do tempo e relações com a dinâmica de fluidos}

O interesse deste tópico é o tratamento da equação de Gross-Pitaevskii dependente do tempo, portanto a função de onda não será escrita somente com a dependência de $\mathbf{r}$, mas também do tempo $t$, ficando da forma $\Psi(\mathbf{r}, t)$. Com a dependência temporal a equação de Gross-Pitaevskii fica da forma:

$$
i \hbar \frac{\partial \Psi(\mathbf{r}, t)}{\partial t}=\left(-\frac{\hbar^{2}}{2 m} \nabla^{2}+V(\mathbf{r})+g_{0}|\Psi(\mathbf{r}, t)|^{2}\right) \Psi(\mathbf{r}, t),
$$

onde $i=\sqrt{-1}$. Para ser consistente com a Eq. 3.15, a função de onda deverá evoluir no tempo com uma fase dada pelo potencial químico, $-\frac{i \mu t}{\hbar}$, ou seja, se a função de onda tiver essa dependência temporal na fase e for aplicada na Eq. 3.26 obteremos a Eq. 3.15.

Essa equação de Schrodinger não-linear pode ser reformulada através de um formalismo hidrodinâmico, representando a função de onda do condensado em termos da fase $S$ e da magnitude $|\Psi(\mathbf{r}, t)|$ :

$$
\Psi(\mathbf{r}, t)=|\Psi(\mathbf{r}, t)| e^{i S(\mathbf{r}, t)} .
$$

Utilizando a definição da densidade de corrente de probabilidade ${ }^{41}$, empregando a relação Eq. 3.27 teremos

$$
\mathbf{j}=\frac{\hbar}{2 m i}\left(\Psi^{*} \nabla \Psi-\Psi \nabla \Psi^{*}\right)=\frac{|\Psi|^{2} \hbar}{m} \nabla S(\mathbf{r}, t)
$$

A usual relação hidrodinâmica $\mathbf{j}=n \mathbf{v}$ (onde $\mathbf{v}$ é o vetor da velocidade) fornecerá:

$$
\mathbf{v}=\frac{\hbar}{m} \nabla S(\mathbf{r}, t)
$$

Por esta relação, com a velocidade sendo proporcional ao gradiente da fase de $\Psi$, concluimos que a velocidade de um superfluido é irrotacional, como assumiu Landau (1947) em seu modelo fenomenologico de dois fluidos proposto para resolver ${ }^{4} \mathrm{He}$, veja Ref. ${ }^{1}$.

Se aplicarmos a Eq. 3.27 na Eq. 3.26 e separarmos as partes reais e imaginárias, teremos as seguintes equações (a da continuidade e a de fluidos quânticos):

$$
\begin{gathered}
\frac{\partial n}{\partial t}+\nabla(n \mathbf{v})=0 \\
\frac{m v^{2}}{2}+V(\mathbf{r})-\frac{\hbar^{2}}{2 m \sqrt{n}} \nabla^{2} \sqrt{n}+g_{0} n=-\hbar \frac{\partial S}{\partial t} .
\end{gathered}
$$

Em primeira aproximação, onde não há potencial externo exercido, tem-se que $\mu=n g_{0}$, o 
que nos permite escrever a Eq. 3.31 sem a dependência de $g_{0}$. Além disso, também temos a propriedade termodinâmica de Gibbs-Duhem $d p=n d \mu$, ou seja, $p=n^{2} g_{0} / 2$, onde $\mathrm{p}$ é pressão. Substituindo essas relações na Eq. 3.31, e tomado o gradiente em ambos lados da igualdade, teremos:

$$
\frac{\partial \mathbf{v}}{\partial t}=-\frac{1}{m n} \nabla p-\nabla\left(\frac{v^{2}}{2}\right)+\frac{1}{m} \nabla\left(\frac{\hbar^{2}}{2 m \sqrt{n}} \nabla^{2} \sqrt{n}\right)-\frac{1}{m} \nabla V(\mathbf{r}),
$$

onde usamos a propriedade dada pela Eq. 3.29. Perceba que a Eq. 3.32 é muito parecida com a de Euler para fluidos dinâmicos ${ }^{1}$

$$
\begin{aligned}
& \frac{\partial \mathbf{v}}{\partial t}+(\mathbf{v} \cdot \nabla) \mathbf{v}+\frac{1}{m n} \nabla p=-\frac{1}{m} \nabla V(\mathbf{r}) \text { ou } \\
& \frac{\partial \mathbf{v}}{\partial t}-\mathbf{v}(\nabla \times \mathbf{v})+\frac{1}{m n} \nabla p+\nabla\left(\frac{v^{2}}{2}\right)=-\frac{1}{m} \nabla V(\mathbf{r}) .
\end{aligned}
$$

Contrastando as equações do superfluido quânticos (Eq.3.32) e clássico (Eq. 3.33), notamos a ausência do termo rotacional na Eq. 3.32, já que ele é zero se não houver singularidades. Já o terceiro termo da Eq. 3.32, também denominado de pressão quântica, não tem correspondente na equação de Euler.

Apesar da descrição física da densidade e velocidade serem diferentes nas condições clássica e quântica, ambas as equações obedecem as leis de conservação de massa (número de partículas) e de momento. 



\section{VÓRTICE}

Gross (1961) e Pitaevskii (1961) introduziram a equação de Gross-Pitaevskii especificamente para descrever vórtices em um BEC. O objetivo deste capítulo é de apresentar a função que descreve o vórtice e a partir dela derivar a densidade, fluxo de velocidade e energia de um condensado com vórtices.

Vamos então considerar que, pela unicidade da função de onda, segue que em um contorno fechado a variação $\Delta S$ na fase da função de onda deve ser múltiplo de $2 \pi$,

$$
\Delta S=\oint \nabla S \cdot d \mathbf{l}=2 \pi q,
$$

onde $q$ é um número inteiro. Dada a definição do campo de velocidade de um superfluido em termos do gradiente da fase da função de onda (Eq. 3.29), podemos calcular a circulação $(\Gamma)$. Essa circulação consiste em uma integral de caminho fechada da velocidade $\mathbf{v}(\mathbf{r})$ conforme segue:

$$
\Gamma=\oint_{C} \mathbf{v}(\mathbf{r}) \cdot d \mathbf{l}
$$

Pelo teorema de Stokes:

$$
\Gamma=\int_{A(C)} \nabla \times \mathbf{v} d \mathbf{A}=2 \pi q \frac{\hbar}{m} .
$$

Considerando um vórtice ao longo do eixo $\hat{\mathbf{z}}$, em coordenadas cilíndricas o campo de velocidade $\mathbf{v}(\rho, \phi)$ será dado por ${ }^{27}$ :

$$
\mathbf{v}(\rho, \phi)=q \frac{\hbar}{m \rho} \hat{\phi}
$$

Observe que esse campo de velocidade tende ao infinito sobre a posição da linha do vórtice. Essa singularidade em $\rho=0$ é que permite que em fluidos quânticos o rotacional de $\mathbf{v}$ seja diferente de zero apenas sobre a posição dos vórtices.

Através do segundo termo da Eq. 4.3, ou se calcularmos o rotacional de Eq. 4.4, teremos que:

$$
\nabla \times \mathbf{v}=\hat{\mathbf{z}} \frac{q h}{m} \delta^{2}(\rho, \phi) .
$$

Para satisfazer a unicidade da função de onda, a função de onda do condensado deve variar como $e^{i q \phi}$, onde $\phi$ é o ângulo azimutal. Tendo como base a Eq. 3.27 e os resultados acima, concluimos que a função de onda de um sistema com um único vórtice pode ser escrita da seguinte forma:

$$
\Psi(\mathbf{r})=f(\rho, \phi, z) e^{i q \phi} .
$$

onde $f(\rho, \phi, z)$ é uma amplitude de valor real. Aplicando a Eq. 4.6 na Eq. 3.7 obteremos o funcional de energia:

$$
E_{s i s}=\int d V\left\{\frac{\hbar^{2}}{2 m}\left[\left(\frac{\partial f}{\partial \rho}\right)^{2}+\left(\frac{\partial f}{\partial z}\right)^{2}\right]+\frac{\hbar^{2}}{2 m} q^{2} \frac{f^{2}}{\rho^{2}}+V(\rho, z) f^{2}+\frac{1}{2} g_{0} f^{4}\right\} .
$$


A diferença desse resultado para o caso de um condensado sem vórtice corresponde predominantemente à energia cinética proveniente da circulação dada pelo campo de velocidade do vórtice $\left(\frac{\hbar^{2}}{2 m} q^{2} \frac{f^{2}}{\rho^{2}}=m \frac{f^{2} v^{2}}{2}\right)$.

A equação para obter a amplitude $(f)$ da função de onda do condensado pode ser obtida aplicando a Eq. 4.6 na equação de Gross-Pitaevskii :

$$
-\frac{\hbar^{2}}{2 m}\left[\frac{1}{\rho} \frac{\partial}{\partial \rho}\left(\rho \frac{\partial f}{\partial \rho}\right)+\frac{\partial^{2} f}{\partial z^{2}}\right]+\frac{\hbar^{2}}{2 m \rho^{2}} q^{2} f+V(\rho, z) f+g_{0} f^{3}=\mu f .
$$

Esta equação será melhor discutida e trabalhada nas seções a seguir.

\subsubsection{Vórtice em um meio uniforme}

Neste seção iremos discutir sobre vórtice em um nuvem de BEC uniforme. Para considerar um meio uniforme, tomamos o potencial externo igual a zero. Considerando novamente a linha de vórtice ao longo do eixo $\hat{z}$, a função de onda do vórtice não depende da coordenada $z$, sendo que a velocidade do fluxo é também azimutal. Para simplificar as contas o valor de $q$ será tomado igual a um, ou seja, trataremos somente de vórtices com um quantum de circulação.

Na Eq. 4.8 pode-se observar que, para grandes distâncias os dois primeiros termos contribuem muito pouco por dependerem de $1 / \rho^{2}$. Perto do eixo do vórtice, porém, a contribuição da energia cinética (os dois primeiros termos) é mais relevante. A distância que equilibra a contribuição, tanto para longe quanto para perto, é $\xi$ dado na Eq. 3.14, o qual caracteriza o "core" (caroço) da linha de vórtice.

As equações Eq. 4.7 e Eq. 4.8 para $V(\rho, z)=0$ e $\frac{\partial^{2} f}{\partial z^{2}}=0$ podem ser simplificadas com a introdução de duas novas variáveis: $x=\rho / \xi$ e $X=f / f_{0}$, onde $\xi=\frac{\hbar}{\sqrt{2 m g_{0} f_{0}^{2}}}$ e $f_{0}=\sqrt{\frac{\mu}{g_{0}}}$, sendo $f_{0}$ a densidade longe do "core". Teremos então o funcional de energia:

$$
E_{s i s}=\int d V \frac{\hbar^{2} f_{0}^{2}}{2 m \xi^{2}}\left[\left(\frac{\partial X}{\partial x}\right)+\frac{X^{2}}{x^{2}}+\frac{X^{4}}{2}\right]
$$

e a correspondente equação dinâmica:

$$
-\frac{1}{x} \frac{\mathrm{d}}{\mathrm{d} x}\left(x \frac{\mathrm{d} X}{\mathrm{~d} x}\right)+\frac{X}{x^{2}}+X^{3}-X=0 .
$$

A solução da Eq. 4.10 pode ser determinada numericamente, e a solução analítica que muito se aproxima da numérica é dada por Ref. ${ }^{1}$ :

$$
X=\frac{x}{\sqrt{2+x^{2}}}
$$

Se tomarmos na Eq. $4.11 \rho \rightarrow 0$ temos $x \rightarrow 0$ e $X \rightarrow 0$ o que significa que a densidade é zero próximo do "core". Já no caso em que $\rho \gg \xi$ temos que $X \rightarrow 1\left(f \rightarrow f_{0}\right)$, ou seja, o vórtice não interfere na densidade da nuvem para grandes distâncias do "core". 
A Eq. 4.9 fornece a energia total do sistema. Se considerarmos que a energia do vórtice é uniforme em $\mathrm{z}$, temos a energia $\varepsilon_{\text {sis }}$ por unidade de comprimento em z dada por:

$$
\varepsilon_{\text {sis }}=\int_{1}^{\rho_{0} / \xi} 2 \pi x d x \frac{\hbar^{2} f_{0}^{2}}{2 m}\left[\left(\frac{\partial X}{\partial x}\right)^{2}+\frac{X^{2}}{x^{2}}+\frac{X^{4}}{2}\right] .
$$

Observe que foi colocado um "cut-off" inferior $\xi$ no limite da integração para evitarmos a divergência logarítimica do segundo termo ${ }^{40}$. Podemos considerar esse cut-off como o "tamanho da singularidade" do vórtice, enquanto que $\rho_{0}$ seria o limite superior dado pela dimensão do sistema.

A energia $\varepsilon_{\text {sis }}$ está associada ao BEC com o vórtice. Porém, para encontrarmos somente a energia associada ao vórtice, considerando a conservação do número de partículas do sistema, subtrairemos de $\varepsilon_{\text {sis }}$ a energia de um BEC sem vórtice. Uma nuvem sem vórtice com o mesmo número de partículas $\tau$ por unidade de comprimento em $z$ contido em um cilindro de raio $D$, mas com densidade $f^{2}$, terá sua energia $\varepsilon_{0}$ por comprimento em $z$ totalmente associada à interação atômica. O número de partículas por unidade de comprimento em $z$ será dado por ${ }^{1}$

$$
\tau=\int_{0}^{D} 2 \pi \rho d \rho f^{2}=\pi D^{2} f_{0}^{2}-\int_{0}^{D} 2 \pi \rho d \rho\left(f_{0}^{2}-f^{2}\right)
$$

Podemos considerar que a energia de nuvem sem vórtice $\varepsilon_{0} \approx \bar{n} g_{0} / 2$, onde $\bar{n}=\tau / \pi D^{2}$ é a média da densidade. Então é possível obter $\varepsilon_{0}$ substituindo $\bar{n}$ por $\tau$ dado pela Eq. 4.13:

$$
\varepsilon_{0}=\frac{1}{2} \pi D^{2} f_{0}^{4} g_{0}-f_{0}^{2} g_{0} \int_{0}^{D} 2 \pi \rho d \rho \cdot\left(f_{0}^{2}-f^{2}\right) .
$$

Os cálculos para obtenção da Eq. 4.14 estão detalhados em citepethick, nestes cálculos o termo $1 / D^{2}$ foi desprezado dado que $D \gg \xi$.

A substituição pelas variáveis adimensionais consideradas na Eq. 4.9 nos dára:

$$
\varepsilon_{0}=\frac{1}{2} \pi D^{2} f_{0}^{4} g_{0}-f_{0}^{4} g_{0} \xi^{2} \int_{0}^{D / \xi} 2 \pi x d x\left(1-X^{2}\right)
$$

Para calcular a energia associada ao vórtice, $\varepsilon_{v}$, basta subtrair a Eq. 4.15 da Eq. 4.12 :

$$
\varepsilon_{v}=\frac{\pi \hbar^{2}}{m} n \int_{0}^{\frac{D}{\xi}} x d x\left[\left(\frac{\partial X}{\partial x}\right)^{2}+\frac{X^{2}}{x^{2}}+\frac{1}{2}\left(1-X^{2}\right)^{2}\right]
$$

A Eq. 4.16 resolvida parcialmente, considerando somente o termo da energia cinética azimutal do vórtice $\left(X^{2} / x^{2}\right)$, resulta em (tomado o valor numérico de $\Xi$ )

$$
\varepsilon_{v} \approx \frac{\pi \hbar^{2}}{m} n \ln \left(\frac{D}{\xi}\right) .
$$

A solução numérica exata da Eq. 4.16 será $^{1}$ :

$$
\varepsilon_{v}=\frac{\pi \hbar^{2}}{m} n \ln \left(1,464 \frac{D}{\xi}\right) .
$$


Considerando $\ln (1,464 D / \xi)=\ln (D / \xi)+\ln (1,464)$ e que $D / \xi \gg 1,464$ ( $D$ é da ordem do raio da nuvem), pois o "core" do vórtice é muito pequeno, vemos que de fato o termo que mais contribui é o $X^{2} / x^{2}$, ou seja, a Eq. 4.17 e a Eq. 4.18 são aproximadamente iguais, de modo que a Eq. 4.17 será considerada a energia de vórtice. Considerando a função analítica aproximada de $X$ (Eq. 4.11) aplicada na Eq. 4.16, o valor de da energia atribuída ao vórtice por unidade de comprimento em $z$ será $\varepsilon_{v} \approx \frac{\pi \hbar^{2}}{m} n \ln \left(1,497 \frac{D}{\xi}\right)$, a qual está muito próxima da solução real Eq. 4.18, Ref. ${ }^{1}$. Logo, está demonstrado que a Eq. 4.11 é uma boa aproximação para a amplitude.

\subsubsection{Vórtice com muitos quanta de energia}

Na Seção anterior foi discutido o caso onde o vórtice tem um quantum de circulação. Nesta Seção iremos tratar o caso onde há vários quanta de energia em um único vórtice.

Como demonstrado na Eq. 4.17, o termo que mais contribui para energia do vórtice no condensado é o advindo da sua energia de circulação, conforme mostrado pela Eq. 4.17. No caso onde $|q|>1$, porém, aparecerá um fator $q^{2}$ multiplicando a Eq. 4.17, já que a energia cinética depende da velocidade ao quadrado e esta é proporcional a $q$. Além disso, o valor de $\xi$, antes proveniente da relação $\frac{\hbar}{2 m \xi^{2}}=n g_{0}$, agora virá da relação $\frac{\hbar q^{2}}{2 m \xi^{2}}=n g_{0}$ pelo mesmo argumento dado acima sobre a energia cinética. Portanto o "novo" $\xi$ aparecerá multiplicado por $q$. Finalmente, podemos escrever a energia aproximada de um vórtice de $q$ quanta de circulação:

$$
\varepsilon_{v} \approx \frac{\pi \hbar^{2} q^{2}}{m} n \ln \left(\frac{D}{\xi q}\right)
$$

É fácil deduzir através da Eq. 4.19 que é inconveniente energeticamente a existência de um único vórtice com muitos quanta para certa quantidade fixa de momento angular total do sistema. Assim sendo, é comum um vórtice de muitos quanta decair em vários de circulação unitária $(q=1)$, formando uma estrutura de rede de vórtices. Se um vórtice de $q$ quanta se decompor em dois de quanta $q_{1}$ e $q_{2}$, com $q=q_{1}+q_{2}$, quanta a diferença de energia entre as configurações final e inicial será atribuída exclusivamente à energia de interação vórtice-vórtice $\varepsilon_{\text {int }}\left(=\varepsilon\left(q_{1}+q_{2}\right)-\varepsilon\left(q_{1}\right)-\varepsilon\left(q_{2}\right)\right)^{1}$ :

$$
\varepsilon_{\text {int }}=\frac{2 \pi q_{1} q_{2} \hbar^{2} n}{m} \ln \frac{D}{d}=\frac{-2 \pi q_{1} q_{2} \hbar^{2} n}{m} \ln \frac{d}{D},
$$

onde $d$ corresponde a distância entre os vórtices, sendo $D \gg d \gg \xi$. Considerando $d \approx \xi$ e $D / \xi \gg 1$ tem-se que:

$$
\varepsilon_{i n t}=\frac{2 \pi q_{1} q_{2} \hbar^{2} n}{m} \ln \frac{D}{\xi}
$$

Verifica-se que essa energia de interação é minimizada quando os vórtices se dispõem em uma rede triangular ${ }^{42-44}$. 
Quando os vórtices interagentes têm circulação oposta $\left(q_{1} q_{2} \leq 0\right)$ a energia da Eq. 4.21 será negativa, o que implica que a força que atua entre os dois vórtices (a qual é menos o gradiente de $\varepsilon_{i n t}$ ) é sempre atrativa, visto que a derivada de $\ln \frac{d}{D}$ com respeito a $d$ é sempre positiva. No caso onde os vórtices têm o mesmo sentido da circulação, a força é repulsiva como no caso de cargas elétricas. Por convenção, o vórtice que rotaciona no sentido anti-horário é tem vorticidade positiva, enquanto que o de sentido horário tem vorticidade negativo.

\subsubsection{Vórtice em uma nuvem armadilhada}

Agora vamos calcular a energia de um vórtice centrado em uma nuvem armadilhada uniforme em $x$ e $y$.

No regime de Thomas-Fermi (TF) temos que $R \gg \xi$. Nas proximidades do vórtice, a energia que mais contribui é a cinética azimutal do vórtice, que depende de $\rho^{-2}$. Podemos definir um raio $\rho_{1}$ tal que $R \gg \rho_{1} \gg \xi$ e que por estar mais perto do "core" do vórtice será onde a energia cinética mais contribuirá em um cilindro de raio $\rho_{2}\left(\rho_{2} \gg \rho_{1}\right)$. A densidade tem comportamento de parábola Eq. 3.20 invertida em nuvens armadilhadas. Em primeira aproximação vamos considerar a densidade de pico constante $n(0)$ para $\rho$ indo de $\xi$ até $\rho_{1}$. Depois calcularemos uma correção nesse termo, considerando que a densidade $n(\rho)$ entre $\rho_{1}$ e $\rho_{2}$ varia como $n(\rho)=n(0)\left(1-\rho^{2} / \rho_{2}^{2}\right)$. Teremos a energia por unidade de comprimento em $z$ corrigida para o vórtice na armadilha (meio não uniforme):

$$
\varepsilon_{v}=\pi n(0) \frac{\hbar^{2}}{m} \ln \left(1,464 \frac{\rho_{1}}{\xi}\right)+\frac{1}{2} \int_{\rho_{1}}^{\rho_{2}} m n(\rho) v^{2}(\rho) 2 \pi \rho d \rho,
$$

onde $v(\rho)=\frac{h}{m} \frac{1}{2 \pi \rho}$. Substituindo $v(\rho)$ e $n(\rho)$ na Eq. 4.22 e resolvendo a integral temos que:

$$
\varepsilon_{v}=\pi n(0) \frac{\hbar^{2}}{m}\left[\left(\ln 1,464 \frac{\rho_{2}}{\xi}\right)-\frac{1}{2}\right] \approx \pi n(0) \frac{\hbar^{2}}{m} \ln \left(0,888 \frac{\rho_{2}}{\xi}\right) .
$$

A integral foi resolvida para $\rho_{2} \gg \rho_{1}$, com os termos de ordem maior do que um para $\rho_{1} / \rho_{2}$ sendo negligenciados. O termo $-1 / 2$ dentro dos colchetes na segunda igualdade na Eq. 4.23 reflete a diminuição da energia cinética causada pela diminuição da densidade de partículas na armadilha harmônica. Da segunda para terceira igualdade da Eq. 4.23 foi utilizado que $1,464 / e^{1 / 2} \approx 0,888$. Perceba que a correção na energia devido ao armadilhamento é muito pequena porque se $\rho_{2} \gg \xi$ então $\ln \left(1,464 \frac{\rho_{2}}{\xi}\right) \gg \frac{1}{2}$ e se desprezar o termo $-\frac{1}{2}$ obtemos a energia do vórtice no plano com densidade $n(0)=n$ e $D=\rho_{2}$.

A energia total do vórtice (agora não mais por unidade de comprimento em $z$ ) pode ser calculada integrando a Eq. 4.23 nos limites de $(-Z, Z)$

$$
E_{v}=\frac{\pi \hbar^{2}}{m} \int_{-Z}^{Z} d z n(0, z) \ln \left[0,888 \frac{\rho(z)}{\xi(z)}\right]
$$


como já argumentado antes (Eq. 3.20) para o caso da armadilha harmônica a densidade no eixo $z$ é parabólica, $n(0, z)=n(0,0)\left(1-\frac{z^{2}}{Z^{2}}\right)$, enquanto que $\rho_{2}=R\left(1-\frac{z^{2}}{Z^{2}}\right)^{\frac{1}{2}} \mathrm{e}$ $\xi(z)=\xi\left[\frac{n(0,0)}{n(0, z))}\right]^{\frac{1}{2}}$. Fazendo essas subtituições na Eq. 4.24 teremos:

$$
\begin{aligned}
E_{v} & =\frac{\pi \hbar^{2} n(0,0)}{m} \int_{-Z}^{Z}\left(1-\frac{z^{2}}{Z^{2}}\right) \ln \left[0,888 \frac{R}{\xi}\left(1-\frac{z^{2}}{Z^{2}}\right)\right] \\
& =4 \pi \frac{n(0,0)}{3} \frac{\hbar^{2}}{m} Z \ln \left[\frac{0,671 R}{\xi}\right] .
\end{aligned}
$$

Concluída a parte que envolve a energia, vamos agora fazer algumas considerações sobre o momento angular. O momento angular $l_{\text {mom }}$ por unidade de comprimento em $z$ é igual a $\hbar$ multiplicado pelo número total de partículas por unidade de comprimento em $z$. Para o caso onde $\rho_{2} \gg \xi$, pode-se considerar a aproximação de Thomas-Fermi, que nos fornece a equação a seguir:

$$
l_{\text {mom }}=n(0) \hbar \int_{0}^{\rho_{2}}\left(1-\frac{\rho^{2}}{\rho_{2}^{2}}\right) 2 \pi \rho d \rho=\frac{1}{2} n(0) \pi \rho_{2}^{2} \hbar .
$$

O momento angular total $L$ proveniente da Eq. 4.26 será:

$$
\begin{aligned}
L & =N \hbar=\hbar \int d \mathbf{r} n(\mathbf{r}) \\
& =n(0,0) \hbar \int_{-Z}^{Z} d z \int_{0}^{\rho_{2}(z)} 2 \pi \rho d \rho\left(1-\frac{\rho^{2}}{R^{2}}-\frac{z^{2}}{Z^{2}}\right) \\
& =\frac{8 \pi}{15} n(0,0) R^{2} Z \hbar .
\end{aligned}
$$

Esses resultados serão utilizados mais adiante na discussão sobre a velocidade angular de rotação crítica da armadilha necessária para gerar um vórtice energeticamente estável.

\subsubsection{Armadilhas que Rotacionam}

Para interações moleculares repulsivas, o estado de menor energia de um condensado de Bose-Einstein armadilhado é geralmente uma superposição de vórtices e excitações elementares, especialmente ondas de superfície. O estado de menor energia é alcançado colocando todo o momento angular no centro de massa.

Para calcularmos a energia $E^{\prime}$ de uma nuvem de átomos rotacionando com velocidade angular de $\Omega$ em termos da energia $E$ do referencial do laboratório (inercial) e do momento angular $L$, podemos utilizar a seguinte equação mecânica:

$$
E^{\prime}=E-\Omega L
$$

Em uma armadilha sem eixo de simetria, o momento angular na direção do eixo de rotação não será conservado. O problema agora é encontrar o estado de menor energia da armadilha, 
ou seja, o menor valor de $E^{\prime}$.

Um estado com componente de momento angular $L$ na direção do eixo de rotação com energia $E_{L}$ será energeticamente favorável a formação de um vórtice se a velocidade angular da armadilha exceder o valor crítico $\Omega_{c}$ Ref. ${ }^{47}$,

$$
\Omega_{c}=\frac{E_{L}-E_{0}}{L},
$$

o qual depende do caráter do estado excitado. $E_{0}$ é a energia da nuvem sem rotação e $E_{L}$ é a energia da nuvem com o vórtice. Um vórtice em uma nuvem onde o número de partículas $N$ seja grande para que a aproximação de TF seja válida terá a energia de um estado com vórtice dada pela Eq. 4.25, ou seja, $E_{L}-E_{0}=E_{v}$, já o momento angular será dado por Eq. 4.27. Aplicando a energia (Eq. 4.25) e o valor do momento angular (Eq. 4.27) podemos obter $\Omega_{c}$ para a formação de um vórtice de um quantum,

$$
\Omega_{c}=\frac{5}{2} \frac{\hbar}{m R^{2}} \ln \left(0,671 \frac{R}{\xi}\right)
$$

De acordo com as Ref. ${ }^{45,46}$ a nuvem continua com formato parabólico mesmo quando rotacionada. Com a adição de mais rotação, cujo $\Omega$ seja múltiplo inteiro $n$ de $\Omega_{c}$, aparecerão mais $n$ vórtices de carga $|q|=1$, ou vórtices de $|q|>1$ de tal forma que a soma das cargas será $n$.

\subsubsection{Um vórtice fora do eixo}

Agora iremos considerar uma linha de vórtice de um único quantum de circulação apontando paralela ao eixo de simetria da armadilha, na direção $z$, a uma distância $b$ do eixo. De acordo com a Eq. 4.17, trocando-se $D$ por $R(z), \xi$ por $\xi(b, z)$ e $n$ por $n(b, z)$, para $q=1$ temos que a energia por unidade de comprimento em $z$ será

$$
\varepsilon_{v}=\frac{\pi \hbar}{m} n(b, z) \ln \left(\frac{R(z)}{\xi(b, z)}\right)
$$

sendo a energia total dada por

$$
E=\frac{\pi \hbar}{m} \int_{-Z_{b}}^{Z_{b}} d z n(b, z) \ln \left(\frac{R(z)}{\xi(b, z)}\right)=E_{0}\left(1-\frac{b^{2}}{R^{2}}\right)^{3 / 2}
$$

onde a energia $E_{0}$ é dada pela Eq. 4.25 e $Z_{b}=Z\left(1-\frac{b^{2}}{R^{2}}\right)^{1 / 2}$ é a extensão da nuvem onde se localiza o vórtice. Na Eq. 4.32 o fator de $\left(1-\frac{b^{2}}{R^{2}}\right)$ é devido a redução da densidade na posição do vórtice, e o outro termo $\left(1-\frac{b^{2}}{R^{2}}\right)^{1 / 2}$ se refere ao decréscimo do comprimento de um vórtice fora do eixo, pois conforme a linha de vórtice se distancia do centro da armadilha, a nuvem fica menos densa e menos extensa.

Esse cálculo demonstra que a energia decresce conforme a distância do vórtice em relação ao eixo da armadilha aumenta. Considerando mecanismos de dissipação que 
possam remover energia da nuvem, o vórtice tenderá a buscar um estado de menor energia, ou seja, migraria para as bordas da nuvem, ver Ref. ${ }^{47}$.

\subsection{Dinâmica de um vórtice armadilhado}

Para estudar a dinâmica de um vórtice armadilhado começamos introduzindo a lagrangiana do sistema em rotação (frequencia $\Omega \hat{z}$ ), cujo potencial de armadilhamento é representado por $V$, Ref. ${ }^{48}$ :

$$
\mathscr{L}(t)=\int d V\left[\frac{i \hbar}{2}\left(\Psi^{*} \frac{\partial \Psi}{\partial t}\right)-\Psi^{*}\left(T-V-\Omega L_{z}\right) \Psi-\frac{1}{2} g_{0}|\Psi|^{4}\right]
$$

sendo $L_{z}$ o momento angular em $z$ e $T$ a parte cinética. Se substituirmos $\Psi(r, t)$ por uma função com parâmetros variacionais, a evolução temporal desses parâmetros corresponderá a dinâmica do condensado. Para isso devemos minimizar a ação relativamente aos parâmetros da função ansatz, e resolver os sistema acoplado de equações de Euler-Lagrange resultantes. Essa função será escolhida da forma Ref. ${ }^{48}$ :

$$
\Psi(r, t)=B(t) f\left[r-r_{0}(t)\right] F\left[r-\eta_{0}(t)\right] \prod_{j=x, y, z} \exp \left[i x_{j} \alpha_{j}(t)+i x_{j}^{2} \kappa_{j}(t)\right]
$$

onde $f(r)$ caracteriza o perfil de densidade da linha de vórtice, enquanto que a função $F(r)$ é a densidade do condensado armadilhado. A função $\eta_{0}(t)=\left(\eta_{0 x}, \eta_{0 y}, \eta_{0 z}\right)$ descreve o movimento do centro de massa do condensado, enquanto que $r_{0}(t)=\left(x_{0}, y_{0}, 0\right)$ descreve a posição do vórtice no plano xy, assumindo que a nuvem tenha formato de disco. $B(t)$ descreverá a evolução da amplitude da nuvem, enquanto que as funções $\alpha_{j}(t)$ e $\kappa_{j}(t)$ descreverão a evolução temporal da fase. Resolvendo o sistema equações acopladas, combinada com a condição de normalização, encontraremos como solução para o movimento do vórtice direcionado em $\hat{\mathbf{z}}$ em uma nuvem armadilhada de formato de disco, isto é, com $R_{x}=R_{y} \gg R_{z}$, devido ao aprisionamento harmônico mais forte na direção $z$ $\left(w_{x}=w_{y} \ll w_{z}\right)$, Ref. ${ }^{41,48,49}$ :

$$
\begin{aligned}
& x_{0}=\beta_{0} R_{x} \sin \left(w_{a} t+\phi_{0}\right), \\
& y_{0}=\beta_{0} R_{y} \cos \left(w_{a} t+\phi_{0}\right),
\end{aligned}
$$

onde $\beta_{0}^{2}=\frac{x_{0}^{2}}{R_{x}^{2}}+\frac{y_{0}^{2}}{R_{y}^{2}}$. Para o deslocamento do centro de massa do condensado teremos:

$$
\begin{aligned}
& \eta_{0 x}=-\frac{15 \beta_{0}}{2 R_{y}} \xi \ln \left(\frac{R_{+}}{\xi}\right) \frac{R_{x}}{R_{x}+R_{y}} \sin \left(w_{a} t+\phi_{0}+\pi\right) \\
& \eta_{0 y}=-\frac{15 \beta_{0}}{2 R_{x}} \xi \ln \left(\frac{R_{+}}{\xi}\right) \frac{R_{y}}{R_{x}+R_{y}} \cos \left(w_{a} t+\phi_{0}+\pi\right),
\end{aligned}
$$


onde $w_{a}=-\frac{3 \hbar w_{x} w_{y}}{4 \mu} \ln \left(\frac{R_{+}}{\xi}\right)$. O deslocamento do centro de massa da nuvem devido a dinâmica do vórtice é quase desprezível pelo fato de que é proporcional ao "core" do vórtice. A quantidade $\beta_{0}$ permanece constante enquanto a linha do vórtice segue uma trajetória elíptica em torno do centro da armadilha, dado que a energia é conservada. $\mathrm{O}$ condensado também precessiona com com mesma frequencia $\omega_{a}$, mas com uma defasagem de $\pi$, ver Fig. 14.

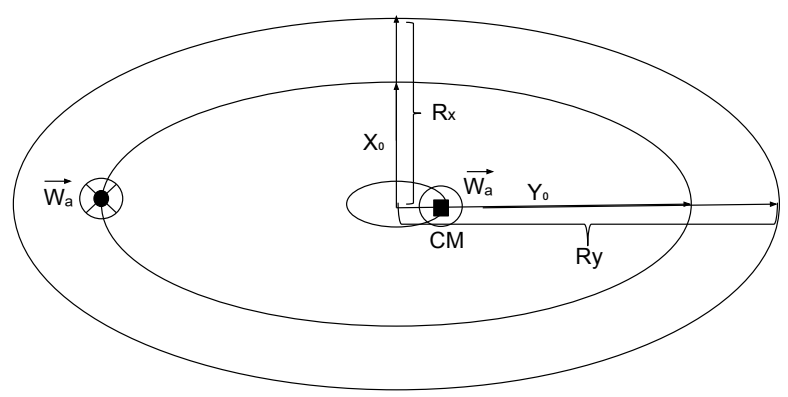

Figura 14: Esquema de um vórtice precessionando em uma nuvem armadilhada com rotação $\Omega$. O vórtice rotaciona com velocidade angular $w_{a}$ enquanto que o centro de massa $(\mathrm{CM})$ do condensado também rotaciona, mas com diferença de fase de $\pi$.

Fonte: Elaborada pelo autor.

\subsection{Interação de um par de vórtices}

Nesta Seção iremos tratar a dinâmica de dois vórtices em um condensado, ambos $\operatorname{com} q$ quanta de momento angular. A Fig. 15 mostra a posição de um vórtice em relação ao plano $2 \mathrm{D}$.

Vamos agora tratar um sistema com vários vórtices. Uma aproximação inicial para a função de onda do sistema é o produto das funções de onda de cada vórtice, onde a função de onda de um vórtice é dada pela Eq. 4.6,

$$
\Psi(\mathbf{r}, 0)=\prod_{i} f\left(\mathbf{d}_{i}\right) e^{ \pm i q \phi_{i}^{\prime}}
$$

onde $\vec{d}_{i}=\vec{r}_{i}-\vec{r}$. Vale observar que a fase toma diferentes sinais dependendo da circulação do vórtice. Além disso, a validade da Eq. 4.39 depende se a posição $r$ seja muito maior do que o "core" do vórtice $\xi$. De acordo com as Ref. ${ }^{50}$, se aplicar a Eq. 4.39 na GPE dependente do tempo, Eq. 3.26, utilizando algumas aproximações (como assumindo o core muito pequeno $\xi^{2} / R^{2} \ll 1$ ), chegaremos na seguinte equação (ansatz) para a função de onda dependente do tempo:

$$
\Psi(\mathbf{r}, t)=\prod_{i} f\left(\mathbf{d}_{i}-\mathbf{u}_{i} t\right) e^{ \pm i q \phi}
$$




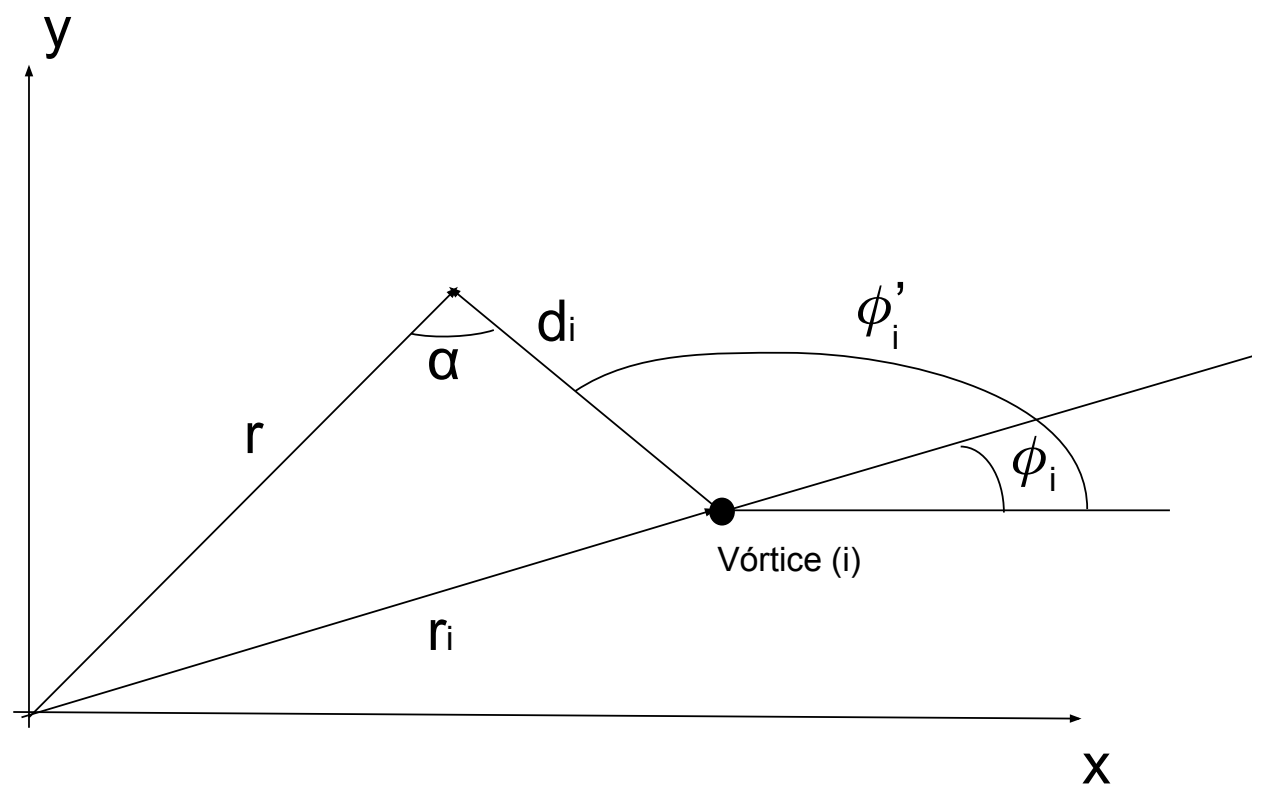

Figura 15: Localização de certo vórtice $i$ na posição $r_{i}$ em um ângulo $\phi_{i}$ atuando em certo ponto determinado por $r$ e $\phi$ a uma distância $d_{i}$.

Fonte: Elaborada pelo autor.

onde $\mathbf{u}_{i}$ corresponde à velocidade de fluxo do i-ésimo vórtice. Ainda nas Ref. ${ }^{50}$, temos que o valor da velocidade translacional do primeiro vórtice depende da velocidade de fluxo dos outros de acordo com a relação:

$$
\mathbf{u}_{i}=\sum_{k \neq i}\left(\frac{q_{k} \hbar}{m}\right) \nabla\left( \pm \phi_{k}^{\prime}\right)
$$

A Eq. 4.41 demonstra que a velocidade translacional de certo vórtice $i$ corresponde a soma do fluxo gerado pelos os outros vórtices $k$ sobre o ponto em que ele está localizado. $\mathrm{O}$ fluxo de velocidade de um vórtice não atua nele mesmo.

De acordo com o esquema ilustrado na Fig. 15, através de propriedades trigonométricas derivamos as relações que seguem:

$$
\begin{gathered}
d_{i}^{2}=r_{i}^{2}+r^{2}-2 r r_{i} \cos \left(\phi-\phi_{i}\right), \\
\frac{d_{i}}{\sin \left(\phi-\phi_{i}\right)}=\frac{r}{\sin \left(\phi^{\prime}-\phi_{i}\right)},
\end{gathered}
$$

que combinadas fornecerão:

$$
\phi^{\prime}=\phi+\arcsin \left(\frac{r_{i} \sin \left(\phi-\phi_{i}\right)}{\sqrt{r^{2}+r_{i}^{2}-2 r r_{i} \cos \left(\phi-\phi_{i}\right)}}\right) .
$$

Vamos então analisar um caso particular com dois vórtices separados de uma distância 2d. No caso em que os vórtices tem sinais opostos e módulo das cargas iguais a 
$1\left(\left|q_{k}\right|=\left|q_{i}\right|=1\right)$, o vórtice 1 de sinal positivo e o vórtice 2 , negativo, vemos através da Eq. 4.41, que esses terão velocidades com sentidos opostos em $\hat{\phi}$, mas sentidos idênticos em $\hat{y}$ já que estão separados por um ângulo $\phi=\pi(\hat{\phi}=\hat{y}$ para $\phi=0$ e $\hat{\phi}=-\hat{y}$ para $\phi=\pi)$. Aplicando a Eq. 4.44 na Eq. 4.41 teremos a velocidade de deslocamento:

$$
-\mathbf{u}_{1}=\mathbf{u}_{2}=\left(\frac{\hbar}{m} \frac{1}{2 d}\right) \hat{\phi}
$$

o qual fará com que os vórtices transladam alinhados na direção $\hat{y}$, ilustrada na situação A da Fig. 16. No caso onde ambos os vórtices possuem sentido positivo, teremos que:

$$
\mathbf{u}_{1}=\mathbf{u}_{2}=\left(\frac{\hbar}{m} \frac{1}{2 d}\right) \hat{\phi}
$$

isto é, os vórtices rotacionarão em $\hat{\phi}$ mantendo o fluxo e o centro de massa do sistema, ilustrado na situação B da Fig. 16.
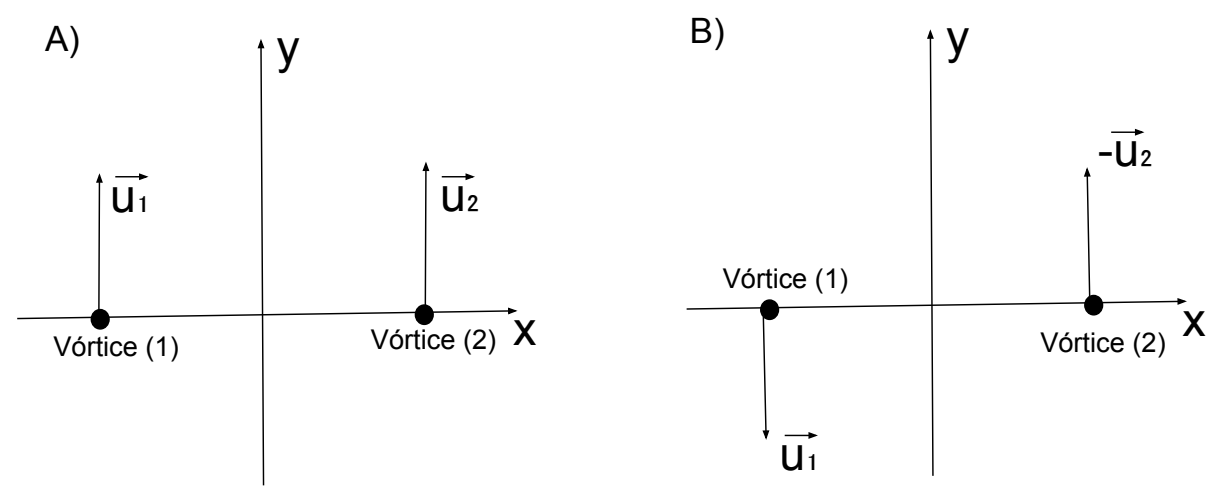

Figura 16: A e B correspondem a vórtices de vorticidades iguais e opostas. Fonte: Elaborada pelo autor.

\subsubsection{Efeito Magnus}

Quando um vórtice sofre a ação de um vetor de uma força o vórtice adquire uma componente extra em sua velocidade na direção pendicular à direção da força aplicada. Para melhor argumentar sobre essa analogia vamos utilizar a energia de interação dada pela Eq.4.20 escrita na forma:

$$
\varepsilon_{\text {int }}=\frac{2 \pi q_{1} q_{2} \hbar^{2} n}{m} \ln \left(\frac{\xi}{d} \frac{D}{\xi}\right)=\frac{2 \pi q_{1} q_{2} \hbar^{2} n}{m}\left[\ln \left(\frac{\xi}{d}\right)+\ln \left(\frac{D}{\xi}\right)\right] .
$$

Vamos definir um potencial de interação, baseado na Eq. 4.47, $\tilde{V}_{i, j}$ para dois vórtices $i$ e $j$ Ref. ${ }^{51}$

$$
\tilde{V}_{i, j}=2 \pi \hbar^{2} n q_{i} q_{j} \frac{1}{m} \ln \left(\frac{1}{r}\right)
$$


onde $\frac{1}{r}=\frac{\xi}{d}$. Perceba que $\tilde{V}_{i, j}$ é o potencial de interação, com $\mathbf{r}$ sendo a distância entre os vórtices em unidades do core $\xi$. Se focarmos em apenas dois vórtices, $i=1$ e $j=2$, as equações de movimento serão Ref. ${ }^{51}$ (considerando que o gradiente do potencial é a força):

$$
\begin{aligned}
& q_{1} \dot{\mathbf{r}}_{1}=-\hat{z} \times \nabla_{1} V_{12}\left(\mathbf{r}_{12}\right), \\
& q_{2} \dot{\mathbf{r}}_{2}=-\hat{z} \times \nabla_{2} V_{21}\left(\mathbf{r}_{21}\right),
\end{aligned}
$$

o sub-índice no gradiente é para informar qual variável o gradiente é tomado com respeito, $\mathbf{r}_{12}$ é vetor entre a posição do vórtice 1 e 2 e $\mathbf{r}_{21}$ é o vetor entre a posição do vórtice 2 e 1. A quantidade $-\nabla_{1} V_{12}\left(\mathbf{r}_{12}\right)$ é a força efetiva $\mathbf{F}_{1}$ que o vórtice 2 exerce no vórtice 1 e a quantidade $-\nabla_{2} V_{21}\left(\mathbf{r}_{21}\right)$ é a força efetiva $\mathbf{F}_{2}$ que o vórtice 1 exerce no vórtice 2 . Podemos reescrever Eq. 4.49 e Eq. 4.50 como

$$
\begin{gathered}
q_{1} \dot{\mathbf{r}}_{1}=\hat{z} \times \mathbf{F}_{1}, \\
q_{2} \dot{\mathbf{r}}_{2}=\hat{z} \times \mathbf{F}_{2}=-\hat{z} \times \mathbf{F}_{1} .
\end{gathered}
$$

Esse sistema mostra que cada vórtice se move perpendicularmente a uma força $\mathbf{F}$. Esse comportamento é chamado de efeito Magnus.

Se calcularmos $\mathbf{F}_{1}$ para $q_{1}=q_{2}=1$ obteremos que $\dot{\mathbf{r}}_{1}$ e $\dot{\mathbf{r}}_{2}$ são idênticos às velocidades dos vórtices dadas por Eq. 4.46. Já no caso $q_{1}=-q_{2}=1$ obteremos $\dot{\mathbf{r}}_{1}$ e $\dot{\mathbf{r}}_{2}$ dados pela Eq. $4.45^{51}$.

Por inspeção ao somarmos cada lado da igualdade das Eq. 4.51 e Eq. 4.52, verificamos que o vetor $q_{1} \dot{\mathbf{r}}_{1}+q_{2} \dot{\mathbf{r}}_{2}$ é zero considerando apenas a interação entre os vórtices. O que implica que $q_{1} \mathbf{r}_{1}+q_{2} \mathbf{r}_{2}$ é constante. Então no caso onde $q_{1}=-q_{2}$ teremos a conservação do espaçamento entre os vórtices.

\subsection{Dinâmica Combinada de Interação Vórtice-Nuvem e Par de Vórtices}

Nesta seção iremos tratar da dinâmica de vórtices ao levar em conta os efeitos da interação vórtice-vórtice e vórtice-nuvem.

As Eq. 4.35 e 4.36 nos fornecem a dinâmica do vórtice ao precessionar devido a interação vórtice-nuvem. As Eq. 4.45 e Eq. 4.46 nos fornecem a dinâmica dos vórtices devido a interação vórtice-anti-vórtice e vórtice-vórtice, respectivamente. Vale lembrar que $\beta_{0}^{2}=\frac{x_{0}^{2}}{R_{x}^{2}}+\frac{y_{0}^{2}}{R_{y}^{2}}$, portanto vemos que as velocidades de precessão crescem conforme o vórtice se aproxima da borda da nuvem, ou seja, para valores maiores do raio $r_{0}=\sqrt{x_{0}^{2}+y_{0}^{2}}$. Já pela Eq. 4.45 vemos que o módulo da velocidade de interação vórtice-vórtice varia inversamente com a distância entre os vórtices.

Assim sendo, podemos concluir que quando os vórtices estão distantes entre si ou próximos da borda da nuvem o efeito vórtice-nuvem prevalecerá. No entanto, quando 
os vórtices estão muito próximos e perto do centro da nuvem a interação vórtice-vórtice prevalecerá. O efeito combinado desses dois regimes gera uma dinâmica de vórtice muito interessante quando tratamos um vórtice e um anti-vórtice confinados em uma armadilha harmônica. $\mathrm{O}$ vórtice e o anti-vórtice quando próximos e no centro da nuvem transladam até a vizinhança da borda o que fará com que as coordenadas dos vórtices representadas por $\left(x_{0}, y_{0}\right)$ aumentem, e consequentemente, a interação vórtice-nuvem passa a predominar. Quando a interação vórtice nuvem predomina, os vórtices rotacionam pela borda da nuvem em sentidos opostos e se aproximam após realizar cada meia revolução. Quando os vórtices se encontram, a distância entre eles diminui o que faz com a interação vórtice-anti-vórtice volte a predominar, e assim eles irão se deslocar até o centro da nuvem retornando a posição inicial. Essa dinâmica é ilustrada com dados experimentais e numéricos na Fig. 17.

(a)

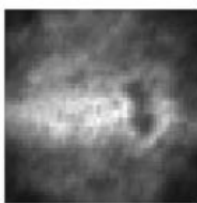

(b)

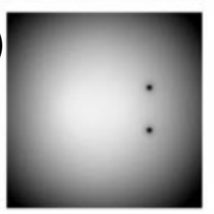

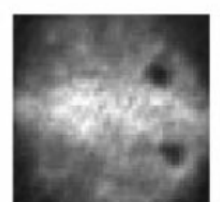

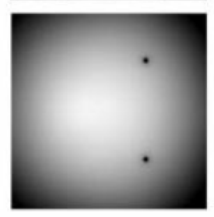

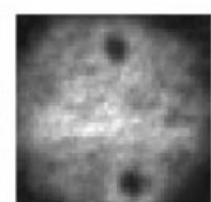

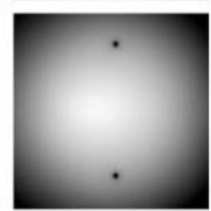

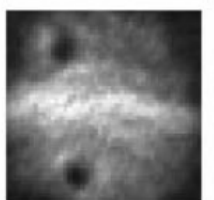

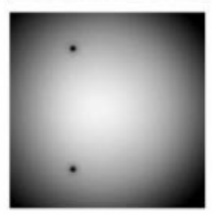

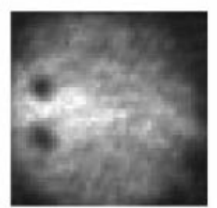

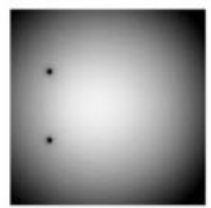

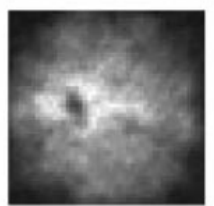
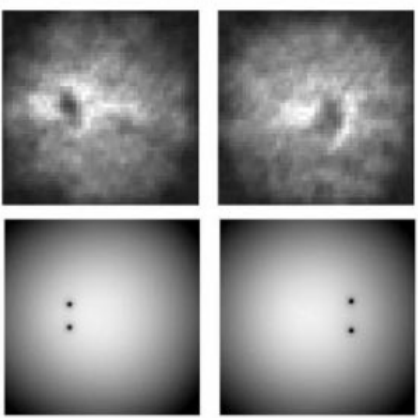

Figura 17: Dinâmica de um vórtice e um anti-vórtice, onde (a) são imagens experimentais e (b) imagens de simulação numérica.

Fonte: NEELY et $a l^{15}$. 



\section{DINÂMICA DE VÓRTICE NA ESFERA}

Nessa Seção iremos estudar vórtices na superfície de uma esfera, isto é, um filme de superfluido com curvatura constante contendo singularidades de vórtices.

A existência de um vórtice de carga positiva sobre essa superfície compacta implica necessariamente na existência de um de carga negativa, com mesma magnitude (quanta) de circulação. A neutralidade da circulação total sobre a superfície da esfera pode ser melhor compreendida quando consideramos as linhas do fluxo de velocidade formada pelo conjunto de vórtices, conforme esquematizado pela Fig. 18. Vemos que a existência de um vórtice positivo implica na formção de um negativo e vice-versa devido às suas linhas de fluxo, ver Ref. ${ }^{10,11}$.

$\mathrm{Na}$ esfera, a única interação que iremos considerar é a interação entre vórtices, pois como a esfera é uma superfície fechada e perfeitamente simétrica, diferentemente de outras superfícies como a Gaussiana, a "sela", entre outras, não há força devido ao seu formato geométrico ${ }^{9}$.

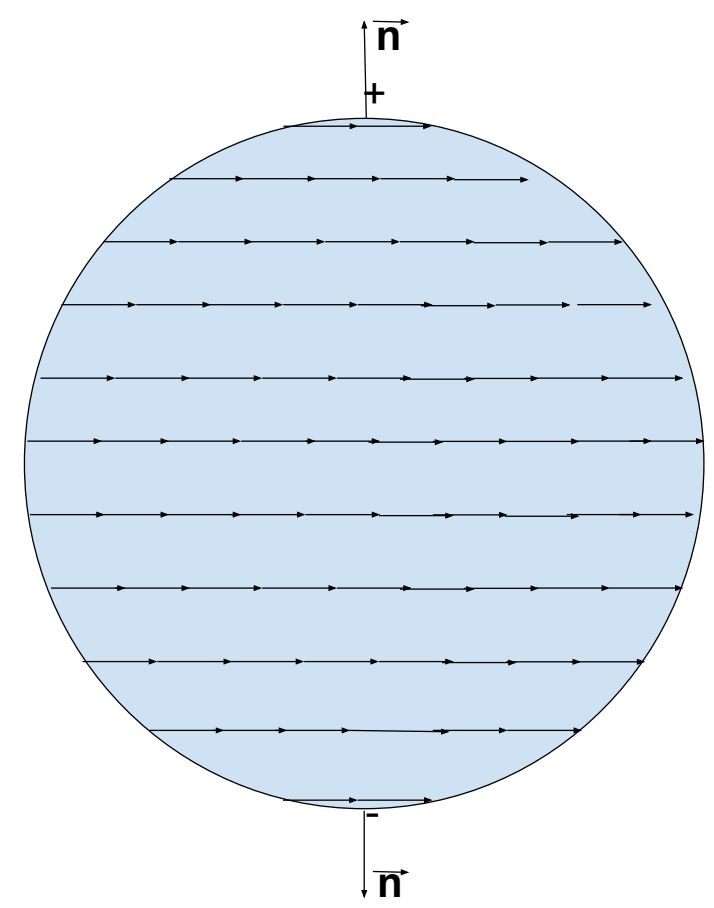

Figura 18: Linhas de velocidade de fluxo de dois vórtices de sinais opostos e separados de $\pi, \vec{n}$ é a normal.

Fonte: Elaborada pelo autor.

Vamos então considerar a casca esférica de raio $R$, cujos pontos são descritos pelas coordenadas polar $\theta$ e azimutal $\phi$. O vetor deslocamento sobre essa superfície será dado por $d \mathbf{s}=R d \theta \hat{\theta}+R \sin \theta d \phi \hat{\phi}$, sendo o vetor normal a superfície $\hat{\mathbf{n}}=\hat{\mathbf{r}}$. 
Como já argumentado antes, o rotacional da velocidade do superfluido é sempre zero quando não há uma singularidade. A equação desta condição para a velocidade v nos fornece que

$$
\hat{\mathbf{n}} \cdot(\nabla \times \mathbf{v})=\frac{1}{R \sin \theta}\left[\frac{\partial\left(\sin \theta v_{\phi}\right)}{\partial \theta}-\frac{\partial v_{\theta}}{\partial \phi}\right]=\frac{q \hbar}{m} 2 \pi \delta^{2}\left(\mathbf{r}-\mathbf{r}_{\mathbf{j}}\right),
$$

considerando a existência de um vórtice em $\mathbf{r}_{\mathbf{j}}$.

Outra propriedade a ser explorada é a incompressibilidade do superfluido descrita pela propriedade $\nabla \cdot \mathbf{v}=0$. Em coordenadas esféricas teremos

$$
\nabla \cdot \mathbf{v}=\frac{1}{R \sin \theta}\left[\frac{\partial}{\partial \theta}\left(\sin \theta v_{\theta}\right)+\frac{\partial v_{\phi}}{\partial \phi}\right]=0 .
$$

Podemos definir um potencial que satisfaz a relação da Eq. 5.2, o qual será denominado de "função de fluxo"12 $\chi$, e que se relaciona com as coordenadas do campo de velocidade segundo as equações:

$$
\begin{gathered}
v_{\theta}=-\frac{q}{R \sin (\theta)} \frac{\partial \chi}{\partial \phi} \\
v_{\phi}=\frac{q}{R} \frac{\partial \chi}{\partial \theta}
\end{gathered}
$$

ou equivalentemente

$$
\mathbf{v}=\frac{q \hbar}{m}(\hat{\mathbf{n}} \times \nabla \chi)
$$

. Escrevendo a Eq. 5.1 em função de $\chi$, teremos a equação de Poisson a ser sastisfeita pela função de fluxo,

$$
\nabla^{2} \chi=\frac{1}{R^{2} \sin \theta} \frac{\partial}{\partial \theta}\left(\sin \theta \frac{\partial \chi}{\partial \theta}\right)+\frac{1}{R^{2} \sin ^{2} \theta} \frac{\partial^{2} \chi}{\partial \phi^{2}}=2 \pi \delta\left(\mathbf{r}-\mathbf{r}_{\mathbf{0}}\right)
$$

Vale ressaltar uma analogia entre o campo de velocidade de um filme fino superfluido com o campo elétrico de uma distribuição de cargas, onde $\chi$ exercerá o papel de um potencial elétrico, e a função delta a de uma carga pontual cujo sinal se equivaleria ao sentido da circulação do vórtice.

Para um líquido irrotacional e incompressível em duas dimensões $(x, y)$, a variável complexa $z=x+i y$, onde $i=\sqrt{-1}$, nos dará a estrutura natural da dinâmica de vórtice. Portanto nos será muito útil introduzir a função complexa $F(z)=\chi(\mathbf{r})+i S(\mathbf{r})$, com $\mathbf{r}=(x, y)$, onde $S$ é dado pelas Eq. 3.27 e Eq. 3.29. Para que possamos ter uma função analítica, as condições de Cauchy-Riemann nos fornecem as seguintes relações entre as derivadas espaciais dos potenciais ${ }^{52}$, as quais estão relacionadas às componentes de velocidade:

$$
v_{x}=\frac{q \hbar}{m} \frac{\partial S}{\partial x}=-\frac{q \hbar}{m} \frac{\partial \chi}{\partial y}
$$




$$
v_{y}=\frac{q \hbar}{m} \frac{\partial S}{\partial y}=\frac{q \hbar}{m} \frac{\partial \chi}{\partial x} .
$$

Essas condições podem ser combinadas na relação:

$$
v_{y}+i v_{x}=\frac{q \hbar}{m} F^{\prime}(z)
$$

Para compreender o uso da notação complexa, vamos aplicá-la para um caso simples, apenas um vórtice de carga igual a $q$ em um plano. Utilizando a função $F=\ln (z)=\ln \left(\rho e^{i q \phi}\right)$ temos

$$
F^{\prime}(z)=\frac{1}{z}=\frac{1}{x+i y}=\frac{x}{x^{2}+y^{2}}-i \frac{y}{x^{2}+y^{2}} .
$$

Se substituirmos as coordenadas $(x, y)$ por $(\rho, \phi)$ através das relações $\cos \phi=$ $\frac{x}{\sqrt{x^{2}+y^{2}}}, \sin \phi=\frac{y}{\sqrt{x^{2}+y^{2}}}$ e $\rho=\sqrt{x^{2}+y^{2}}$, a Eq. 5.10 assume a forma

$$
F^{\prime}=\frac{\cos \phi}{\rho}-i \frac{\sin \phi}{\rho}
$$

Pela relação Eq. 5.9 temos $v_{y}=\frac{q \hbar}{m} \frac{\cos \phi}{\rho}$ e $v_{x}=-\frac{q \sin \phi}{\rho} x$. Finalmente podemos deduzir o fluxo de velocidade $\mathbf{v}=v_{x} \hat{\mathbf{x}}+v_{y} \hat{\mathbf{y}}=\frac{q \hbar}{m}[\cos \phi \hat{\mathbf{y}}-\sin \phi \hat{\mathbf{x}}]=\frac{q \hbar}{m \rho} \hat{\phi}$, idêntico a Eq. 4.4. Se o vórtice estiver deslocado em $z_{0}$ a função $F$ é dada por $F=\ln \left(z-z_{0}\right)^{12}$.

\subsection{Projeção estereográfica para dois vórtices na esfera}

É possível realizar uma transformação conforme ${ }^{3}$ tal que uma esfera seja projetada em um plano complexo. Se projetarmos os vórtices localizados em uma esfera sobre um plano, será possível encontrar a dinâmica destes resolvendo para o plano e usando a solução na esfera.

Na projeção estereográfica (transformação conforme), cada ponto da esfera (com excessão do ponto no extremo sul) tem uma correspondência unívoca com os pontos sobre um plano tangente a essa esfera no polo norte ${ }^{13}$. Um ponto sobre a esfera com coordenadas $(\theta, \phi)$ teria sua localização no plano dada pela coordenada complexa $z=\rho e^{-i \phi}$, onde $\rho=2 R \tan \theta / 2$. Vemos que a projeção estereográfica é uma transformação conforme onde associamos um círculo de raio $\rho$ sobre o plano complexo com um círculo de ângulo polar $\theta=2 \arctan [\rho /(2 R)]$ e $0 \leq \phi \leq 2 \pi$ na esfera unitária. O ângulo $\phi$ no plano coincide com o ângulo azimutal $\phi$ na esfera ${ }^{13,14}$.

Para solucionarmos o caso em que temos dois vórtices de sinais opostos $q_{1}=-q_{2}=1$ projetados no plano complexo em $z_{ \pm}=2 R \tan \left(\theta_{ \pm} / 2\right) e^{i \phi_{ \pm}}$, onde $\left(\theta_{ \pm}, \phi_{ \pm}\right)$são as coordenadas dos vórtices de cargas $q= \pm 1$ devemos utilizar a função $F=\ln \left(z-z_{+}\right)-\ln \left(z-z_{-}\right)$, a qual é a solução de dois vórtices deslocados do eixo em $z_{+}$e $z_{-}$no plano. Se substituirmos o valor de $z$ em $F$ obteremos $F=\ln \left(\frac{2 R \tan (\theta / 2) e^{i \phi}-z_{+}}{2 R \tan (\theta / 2) e^{i \phi}-z_{-}}\right)$. A função de fluxo correspondente 


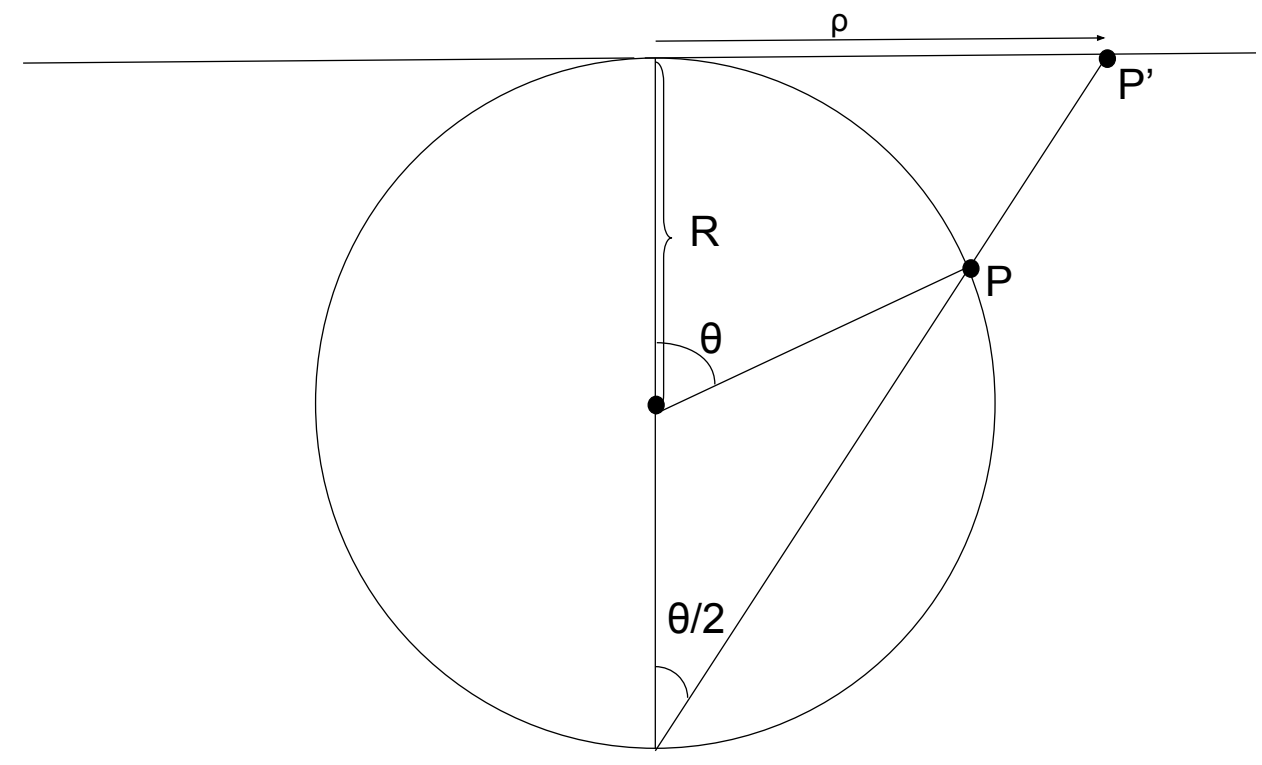

Figura 19: Projeção estereográfica da esfera no plano. Na figura podemos observar a projeção de um ponto qualquer P.

Fonte: Elaborada pelo autor.

será dada por $\chi=\operatorname{Re}[F]$, que fornecerá a função de fluxo do par de vórtices (ver apêndice $\mathrm{G})$ :

$$
\chi_{s}(\theta, \phi)=\frac{1}{2} \ln \left[\frac{\tan ^{2}(\theta / 2)+\tan ^{2}\left(\theta_{+} / 2\right)-2 \tan (\theta / 2) \tan \left(\theta_{+} / 2\right) \cos \left(\phi-\phi_{+}\right)}{\tan ^{2}(\theta / 2)+\tan ^{2}\left(\theta_{-} / 2\right)-2 \tan (\theta / 2) \tan \theta_{-} / 2 \cos \left(\phi-\phi_{-}\right)}\right]
$$

A Eq. 5.12 pode ser escrita como a soma da função de fluxo de cada um dos vórtices $\chi_{s}=\chi_{+}-\chi_{-}$. Considerando a função de fluxo de um dos vórtices $\chi_{j}(j= \pm)$, podemos utilizar a relação $\tan \theta / 2=\sqrt{\frac{1-\cos \theta}{1+\cos \theta}}$ para reescrevê-la na forma

$$
\chi_{j}(\theta, \phi)=\frac{1}{2} \ln \left[\frac{2-2 \cos \theta \cos \theta_{j}-2 \sin \theta \sin \theta_{j} \cos \left(\phi-\phi_{j}\right)}{(1+\cos \theta)\left(1+\cos \theta_{j}\right)}\right] .
$$

Como nosso maior interesse é achar a velocidade de fluxo, a qual depende do gradiente de $\chi$ então o termo $\left(1-\cos \theta_{j}\right)$ no denomidor dentro do logaritmo na Eq. 5.13 será anulado pelo gradiente. Além disso, a velocidade de fluxo corresponde à soma das velocidades de fluxo dos vórtices na esfera, e como eles têm sinais oposto o termo $(1+\cos \theta)$ também localizado no denominador dentro do logaritmo na Eq. 5.13 será anulado. Portanto, se a "função de fluxo" for escrita na forma abaixo teremos a mesma velocidade de fluxo total,

$$
\chi_{j}(\theta, \phi)=\frac{1}{2} \ln \left[2-2 \cos \theta \cos \theta_{j}-2 \sin \theta \sin \theta_{j} \cos \left(\phi-\phi_{j}\right)\right] .
$$


, teremos a mesma velocidade. Considerando agora o ângulo $\gamma_{j}$ entre os vetores $\mathbf{r}$ e $\mathbf{r}_{j}$, isto é, $\hat{\mathbf{r}} \cdot \hat{\mathbf{r}}_{j} \equiv \cos \gamma_{j}=\sin \theta \sin \theta_{j} \cos \left(\phi-\phi_{j}\right)+\cos \theta \cos \theta_{j}$, com a identidade $1-\cos \gamma_{j}=$ $2 \sin ^{2}\left(\gamma_{j} / 2\right)$ a Eq. 5.14 ficará da forma

$$
\chi_{j}\left(\hat{\mathbf{r}}, \hat{\mathbf{r}}_{j}\right)=\frac{1}{2} \ln \left[4 \sin ^{2}\left(\gamma_{j} / 2\right)\right] .
$$

Obtida a função de fluxo de um vórtice de coordenada $\mathbf{r}_{j}=\left(R, \theta_{j}, \phi_{j}\right)$, podemos derivar o fluxo de velocidade correspondente em um ponto arbitrário $\theta, \phi$ sobre a superfície da esfera, aplicando a relação Eq. 5.5

$\mathbf{v}_{j}(\mathbf{r})=\frac{\hbar}{m} \frac{q_{j}}{4 R \sin ^{2}\left(\gamma_{j} / 2\right)}\left\{\left[\cos \theta_{j} \sin \theta-\cos \theta \sin \theta_{j} \cos \left(\phi-\phi_{j}\right)\right] \hat{\phi}-\left[\sin \theta_{j} \sin \left(\phi-\phi_{j}\right)\right] \hat{\theta}\right\}$,

onde

$$
\sin \left(\frac{\gamma_{j}}{2}\right)=\left[\frac{1-\sin \theta_{j} \sin \theta \cos \left(\phi-\phi_{j}\right)-\cos \theta_{j} \cos \theta}{2}\right]^{1 / 2} .
$$

Por outro lado, o campo de velocidades na posição $\mathbf{r}(R, \theta, \phi)$ devido a um par de cargas $q_{i}$ e $q_{j}$, nas posições $\mathbf{r}_{i}$ e $\mathbf{r}_{j}$ respectivamente, será dada por $\mathbf{v}(\mathbf{r})=\mathbf{v}_{+}(\mathbf{r})+\mathbf{v}_{-}(\mathbf{r})$. Observe que a Eq. 5.16 é o equivalente da Eq. 4.4.

\subsection{Energia de interação}

Como já argumentado anteriormente, a contribuição do vórtice na energia total do sistema é predominantemente cinética

$$
E_{k i n}=\frac{n}{2} \int_{A} d^{2} r m \mathbf{v}^{2},
$$

onde $A$ é toda a area da superfície e $\mathbf{v}$ é soma das velocidades de fluxo gerado pelos vórtices. Logo temos que:

$$
\begin{aligned}
E_{k i n} & =\frac{n}{2} \int_{A} d^{2} r m\left(\mathbf{v}_{i}+\mathbf{v}_{j}\right)^{2} \\
& =\frac{n}{2} m R^{2} \int_{\epsilon}^{\pi-\epsilon} \sin \theta d \theta \int_{-\pi}^{\pi} d \phi\left[\mathbf{v}_{i}^{2}+\mathbf{v}_{j}^{2}+2 \mathbf{v}_{i} \cdot \mathbf{v}_{j}\right],
\end{aligned}
$$

onde $\epsilon \approx \frac{\xi}{R}$ trata-se da abertura angular correspondente ao core do vórtice. Os dois primeiros termos do integrando se referem a energia gerada por cada vórtice separadamente e o terceiro à interação entre os vórtices. Começando com os dois primeiros termos da Eq. 5.19 temos (utilizando a Eq. 5.16):

$$
\begin{aligned}
& E_{i}=\frac{n}{2} \int d^{2} r m \mathbf{v}_{i}^{2}=\left.\frac{q_{i}^{2} \pi n \hbar^{2}}{4 m}[\cos \theta+4 \ln \sin (\theta / 2)]\right|_{\epsilon} ^{\pi}=\frac{q_{i}^{2} \pi n \hbar^{2}}{M}\left[\ln \left(\frac{2 R}{\xi}\right)-\frac{1}{2}\right], \\
& E_{j}=\frac{n}{2} \int d^{2} r m \mathbf{v}_{j}^{2}=\left.\frac{q_{j}^{2} \pi n \hbar^{2}}{4 m}[\cos \theta+4 \ln \sin (\theta / 2)]\right|_{\epsilon} ^{\pi}=\frac{q_{j}^{2} \pi n \hbar^{2}}{m}\left[\ln \left(\frac{2 R}{\xi}\right)-\frac{1}{2}\right] .
\end{aligned}
$$


Vemos que esse resultado independe da posição dos vortices sobre a superficie da esfera, assim como do sentido da circulação. Já o terceiro termo da Eq. 5.19 será

$$
E_{\text {int }}=\frac{n}{2} m R^{2} \int_{0}^{2 \pi} d \phi \int_{\epsilon}^{\pi-\epsilon} 2 \mathbf{v}_{i} \cdot \mathbf{v}_{j} \sin \theta d \theta .
$$

Se recuperarmos $d^{2} r$ podemos fazer algumas manipulações na integral da Eq. 5.22:

$$
\begin{aligned}
\int_{\epsilon}^{\pi-\epsilon} \mathbf{v}_{i} \cdot \mathbf{v}_{j} \sin \theta d \theta & =\frac{q_{i} q_{j} \hbar^{2}}{m^{2}} \int_{\epsilon}^{\pi-\epsilon} \nabla \chi_{i} \cdot \nabla \chi_{j} \sin \theta d \theta \\
& =\frac{q_{i} q_{j} \hbar^{2}}{m^{2}} \int_{\epsilon}^{\pi-\epsilon}\left[\nabla \cdot\left(\chi_{i} \nabla_{j}\right) \chi_{j}-\chi_{i} \nabla^{2} \chi_{j}\right] \sin \theta d \theta
\end{aligned}
$$

Na última igualdade da Eq. 5.23 o primeiro termo integrado será zero por causa da periodicidade da esfera, enquanto que no segundo podemos aplicar a identidade $\nabla^{2} \chi_{j}=$ $2 \pi \delta\left(\mathbf{r}-\mathbf{r}_{\mathbf{j}}\right)$. Assim sendo, a Eq. 5.23 poderá ser reescrita como:

$$
E_{i n t}=-\frac{2 \pi n \hbar^{2}}{m} q_{i} q_{j} \chi_{i, j}
$$

onde $\chi_{i, j}=\chi_{j}\left(\hat{\mathbf{r}}_{\mathbf{i}}, \hat{\mathbf{r}_{\mathbf{j}}}\right)$ é dado pela 5.15. Por isso temos que a $E_{\text {int }}$ será

$$
E_{\text {int }}=-\pi \frac{n \hbar^{2}}{m} q_{i} q_{j} \ln \left(\frac{2 R \sin \left(\gamma_{i, j} / 2\right)}{\xi}\right) .
$$

Se tivermos $N$ pares podemos generalizar a $E_{\text {int }}$ dada pela Eq. 5.24,

$$
E_{i n t}^{N}=-\frac{\pi n \hbar^{2}}{m} \sum_{i \neq j}^{2 N} q_{i} q_{j} \chi_{i, j} .
$$

De acordo com Eq. 5.26, sendo $q_{i} q_{j}<0$, quando os vortices se aproximam, ou seja, $\gamma_{i, j} \rightarrow 0$, teremos $E_{\text {int }} \rightarrow-\infty$.

\subsection{Dinâmica de um par de vórtice na casca esférica}

Nesta seção iremos discutir a dinâmica de um dipolo de vórtice.

Como já discutido a velocidade de um vórtice corresponde à soma vetorial das velocidades de fluxo gerado pelos outros vórtices (Eq. 4.41). Dessa forma o módulo da velocidade de um vórtice na posição $\mathbf{r}_{i}$ devido a um vórtice localizado na posição $\mathbf{r}_{j}$ será:

$$
\begin{aligned}
\left|\mathbf{v}_{j \rightarrow i}\left(\mathbf{r}_{i}\right)\right| & =\frac{\hbar}{m} \frac{1}{4 R \sin ^{2}\left(\gamma_{i, j} / 2\right)} \sqrt{\left[\sin \theta_{i} \cos \theta_{j}-\cos \theta_{i} \sin \theta_{j} \cos \left(\phi_{i}-\phi_{j}\right)\right]^{2}+\left[\sin \theta_{j} \sin \left(\phi_{i}-\phi_{j}\right)\right]^{2}} \\
& =\frac{\hbar}{m} \frac{1}{4 R \sin ^{2}\left(\gamma_{i, j} / 2\right)} \sqrt{1-\cos ^{2} \gamma_{j, i}} \\
& =\frac{\hbar}{m} \frac{1}{2 R} \cot \left(\frac{\gamma_{j, i}}{2}\right),
\end{aligned}
$$

ou seja, a velocidade de fluxo depende puramente da cotangente do ângulo entre o vórtice e o ponto a ser analisado. 
Vamos assumir dois vórtices de cargas opostas em posições quaisquer. Devido a simetria da esfera podemos adequar o sistema de coordenadas para que ambos estejam em $\phi=0$, com o vórtice positivo em $\theta_{+}=(\pi / 2-\delta \theta)$ e o vórtice negativo em $\theta_{-}=(\pi / 2+\delta \theta)$, conforme ilustrado na Fig. 20. De acordo com a Eq. 5.27 temos que

$$
\begin{aligned}
& \mathbf{v}_{+\rightarrow-}=\frac{\hbar}{2 R m} \cot (\delta \theta) \hat{\phi}, \\
& \mathbf{v}_{-\rightarrow+}=\frac{\hbar}{2 R m} \cot (\delta \theta) \hat{\phi} .
\end{aligned}
$$

. As relações anteriores permitem concluir que os vortices transladarão paralelamente ao

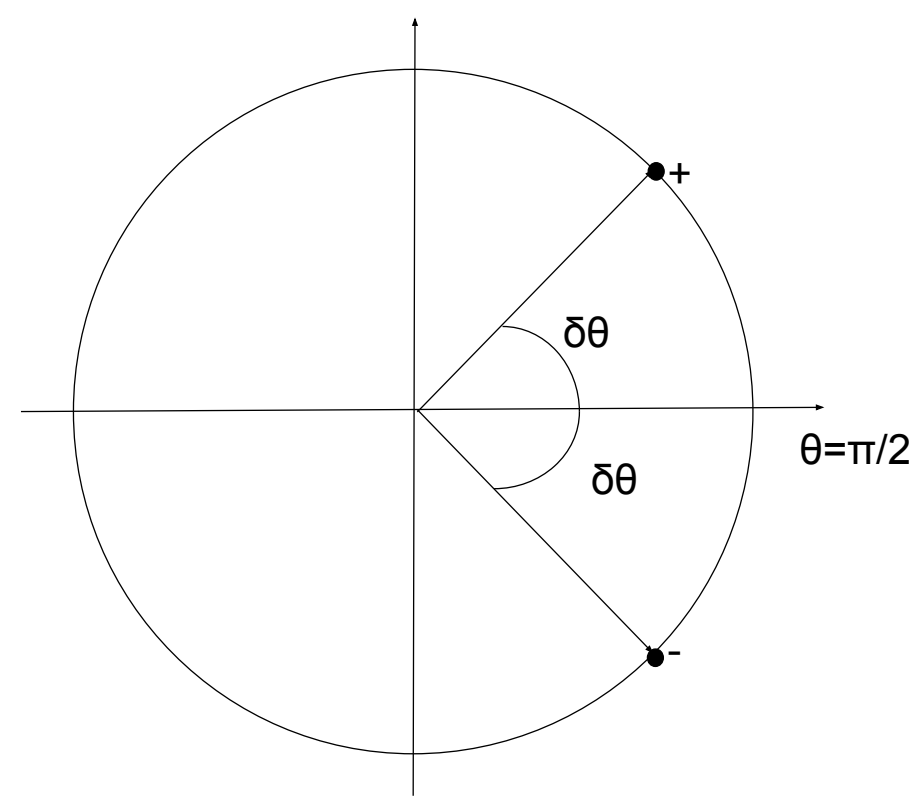

Figura 20: Par de vórtices em uma posição qualquer enquanto que os eixos adotados são tais que ambos estão em $\phi=0$ e $\theta=\pi / 2$ coincide com o ponto médio entre eles.

Fonte: Elaborada pelo autor.

círculo maior da esfera, mantendo fixa a distância inicial entre eles.

\subsubsection{Dinâmica de dois pares de vórtices na esfera}

Iremos analisar alguns casos com mais de um par de vórtices, começando com o caso simétrico de dois pares idênticos, alinhados com suas cargas antisimetricamente (caso A) ou simetricamente (caso B) posicionadas, ver Fig. 21. Em ambas situações (A e B) um par se encontra no plano $\phi=0$ e o outro em $\phi=\pi$, e com os seus centros (ponto médio da cada par) deslocados de $(\Delta \theta+\delta \theta)$ do polo norte da esfera. Consideraremos os vórtices 
enumerados de 1 a 4, com numeração indicando a ordem crescente no sentido horário da esfera.

A velocidade $\mathbf{v}_{i \rightarrow j}$ é definida como a velocidade do vórtice $j$ devido a influência (interação) do vórtice $i$.

Vamos agora analisar a situação A. O vórtice número 3, localizado em $\left(\theta_{3}, \phi_{3}\right)=$
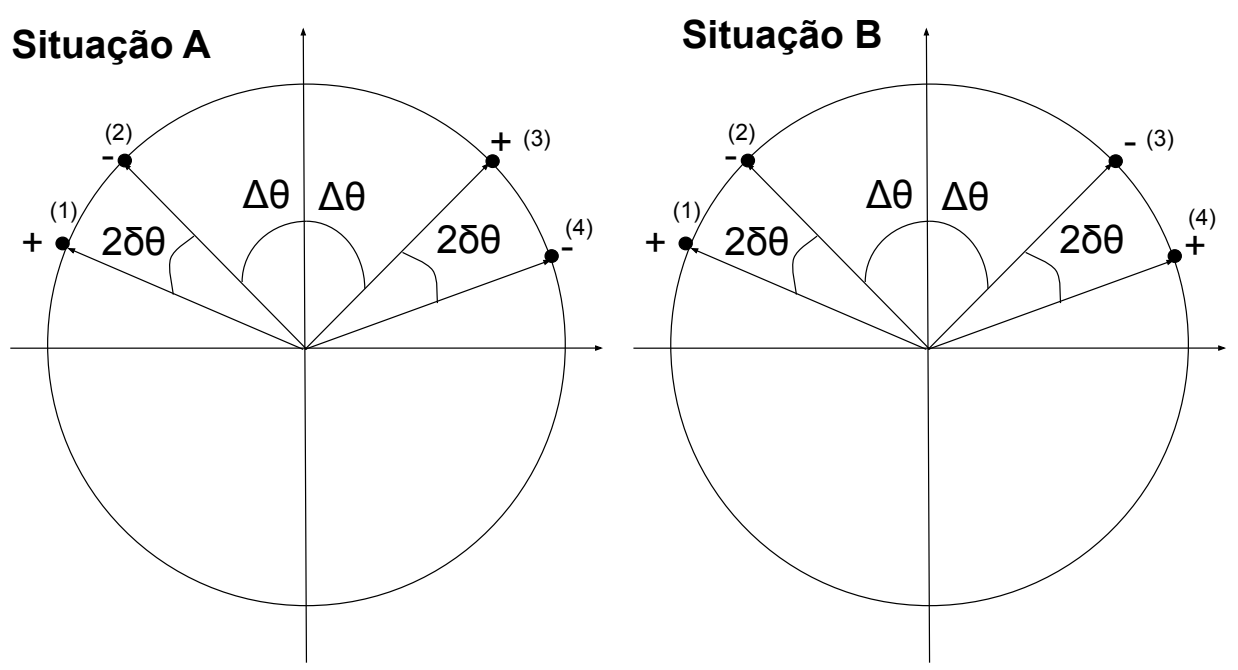

Figura 21: As situações propostas A e B esquematizam os dipolos de vórtices dispostos simetricamente e antisimetricamente respectivamente.

Fonte: Elaborada pelo autor.

$(\Delta \theta, 0)$, está sob ação das velocidades de fluxo $\mathbf{v}_{1 \rightarrow 3}$, devido ao vórtice 1 localizado em $\left(\theta_{1}, \phi_{1}\right)=(\Delta+2 \delta, \pi) ; \mathbf{v}_{2 \rightarrow 3}$, devido ao vórtice 2 localizado em $\left(\theta_{2}, \phi_{2}\right)=(\Delta \theta, \pi)$ e $\mathbf{v}_{4 \rightarrow 3}$ devido ao vórtice 4 localizado em $\left(\theta_{4}, \phi_{4}\right)=(\Delta \theta+2 \delta \theta, 0)$. Perceba pela Eq. 5.16 que nenhuma das velocidades de fluxo sobre o vórtice 3 possui componente em $\hat{\theta}$, pois estão todos nos planos de $\phi=0$ ou $\phi=\pi$. Isso implica que o módulo da velocidade dada pela Eq. 5.27 será apenas na direção $\hat{\phi}$. Concluimos que $\mathbf{v}_{3}$ será:

$$
\mathbf{v}_{3}=\sum_{i, i \neq 3}^{4} \mathbf{v}_{i \rightarrow 3}=\frac{\hbar}{2 m R}[\cot (\delta \theta+\Delta \theta)+\cot (\delta \theta)-\cot (\Delta \theta)] \hat{\phi} .
$$

Se fizermos os mesmos cálculos para obtermos as $\mathbf{v}_{1}, \mathbf{v}_{2}$ e $\mathbf{v}_{4}$ teremos:

$$
\begin{gathered}
\mathbf{v}_{1}=\sum_{i, i \neq 1}^{4} \mathbf{v}_{i \rightarrow 1}=-\frac{\hbar}{2 m R}[\cot (\delta \theta)-\cot (\delta \theta+\Delta \theta)+\cot (\Delta \theta+2 \delta \theta)] \hat{\phi} \\
\mathbf{v}_{2}=\sum_{i, i \neq 2}^{4} \mathbf{v}_{i \rightarrow 2}=-\frac{\hbar}{2 m R}[-\cot (\Delta \theta)+\cot (\delta \theta+\Delta \theta)+\cot (\delta \theta)] \hat{\phi} \\
\mathbf{v}_{4}=\sum_{i, i \neq 4}^{4} \mathbf{v}_{i \rightarrow 4}=\frac{\hbar}{2 m R}[\cot (\delta \theta)-\cot (\delta \theta+\Delta \theta)+\cot (\Delta \theta+2 \delta \theta)] \hat{\phi}
\end{gathered}
$$

Perceba que $\mathbf{v}_{1}=-\mathbf{v}_{4}$ e que $\mathbf{v}_{3}=-\mathbf{v}_{2}$, o que somado ao fato de que os vórtices 1 e 2 estão em $\phi=\pi$ e os 3 e 4 estão em $\phi=0$, podemos concluir que os vórtices irão girar 
em torno do eixo $\theta=\pi / 2$ desde que o período de revolução do vórtice 1 seja igual ao do vórtice 2 . O vórtice 1 deve percorrer a distância de $2 \pi R \cos (\Delta \theta+2 \delta \theta)$ no mesmo tempo em que o vórtice 2 percorre $2 \pi R \cos (\Delta \theta)$ :

$$
\frac{2 \pi R \cos (\Delta \theta)}{2 \pi R \cos (\Delta \theta+\delta \theta)}=\frac{\left|\mathbf{v}_{2}\right|}{\left|\mathbf{v}_{1}\right|}=\frac{\left|\mathbf{v}_{3}\right|}{\left|\mathbf{v}_{4}\right|}
$$

ou seja,

$$
\frac{\cos \Delta \theta}{\cos (\Delta \theta+\delta \theta)}=\frac{|-\cot (\Delta \theta)+\cot (\Delta \theta+\delta \theta)+\cot \delta \theta|}{|\cot \delta \theta-\cot (\Delta \theta+\delta \theta)+\cot \Delta \theta+2 \delta \theta|}
$$

deva ser satisfeita para os vórtices rotacionarem. Caso contrário sairão do mesmo plano em $\phi$ aparecendo componente $\hat{\theta}$ e o movimento será de 4 corpos interagentes, o que é bastante complexo. Não há solução para a Eq. 5.35, mas se tomarmos $\delta \theta=\Delta \theta=\pi / 4$ vemos das Eq. 5.30 até Eq. 5.33, que as velocidades dos vórtices são todas nulas, ou seja, nesta configuração diamentralmente opostas os vórtices ficarão estáticos.

$\mathrm{Na}$ situação B os vórtices 3 e 4 invertem de sinal e, assim, as velocidades de fluxo que atuam em 1, 2 e entre eles mesmos também irão inverter o sinal. Logo para este caso temos que, pela Eq. 5.16,

$$
\begin{gathered}
\mathbf{v}_{1}=\sum_{i, i \neq 1}^{4} \mathbf{v}_{i \rightarrow 1}=-\frac{\hbar}{2 m R}[-\cot (\delta \theta)-\cot (\delta \theta+\Delta \theta)+\cot (\Delta \theta+2 \delta \theta)] \hat{\phi} \\
\mathbf{v}_{2}=\sum_{i, i \neq 2}^{4} \mathbf{v}_{i \rightarrow 2}=-\frac{\hbar}{2 m R}[-\cot (\Delta \theta)+\cot (\delta \theta+\Delta \theta)-\cot (\delta \theta)] \hat{\phi} \\
\mathbf{v}_{3}=\sum_{i, i \neq 3}^{4} \mathbf{v}_{i \rightarrow 3}=\frac{\hbar}{2 m R}[-\cot (\delta \theta+\Delta \theta)+\cot (\delta \theta)+\cot (\Delta \theta)] \hat{\phi} \\
\mathbf{v}_{4}=\sum_{i, i \neq 4}^{4} \mathbf{v}_{i \rightarrow 4}=\frac{\hbar}{2 m R}[\cot (\delta \theta)+\cot (\delta \theta+\Delta \theta)-\cot (\Delta \theta+2 \delta \theta)] \hat{\phi}
\end{gathered}
$$

Perceba que $\mathbf{v}_{1}=\mathbf{v}_{4}$ e que $\mathbf{v}_{3}=\mathbf{v}_{2}$. Sendo assim podemos concluir que os vórtices irão rotacionar em torno do eixo $\theta=0$ desde que o período de revolução do vórtice 1 seja igual ao do vórtice 2 . O vórtice 1 deve percorrer a distância de $2 \pi R \sin (\Delta \theta+2 \delta \theta)$ no mesmo tempo em que o vórtice 2 percorrerá $2 \pi R \sin (\Delta \theta)$. Na situação $\mathrm{B}$ teremos que

$$
\frac{2 \pi R \sin \Delta \theta}{2 \pi R \sin (\Delta \theta+\delta \theta)}=\frac{\left|\mathbf{v}_{2}\right|}{\left|\mathbf{v}_{1}\right|}=\frac{\left|\mathbf{v}_{3}\right|}{\left|\mathbf{v}_{4}\right|}
$$

ou seja,

$$
\frac{\sin \Delta \theta}{\sin (\Delta \theta+\delta \theta)}=\frac{-\cot (\Delta \theta)+\cot (\Delta \theta+\delta \theta)-\cot \delta \theta}{-\cot (\Delta \theta+\delta \theta)-\cot (\delta \theta)+\cot (\Delta \theta+2 \delta \theta)} .
$$

A Eq. 5.41 será sempre satisfeita se $\delta \theta=\pi / 2-\Delta \theta$. Neste caso, as posições dos vórtices 1 , 2,3 e 4 serão $(\pi / 2+\delta \theta, \pi),(\pi / 2-\delta \theta, \pi),(\pi / 2-\delta \theta, 0)$ e $(\pi / 2+\delta \theta, 0)$, respectivamente. Esse seria o caso onde os pares de vórtices estão simétricos em relação ao eixo $\theta=\pi / 2$ e rotacionarão em torno deste eixo. 


\subsection{Minimização de energia de dipolos}

Nesta Seção iremos tratar a energia de interação de mais de um dipolo. No entanto, no intuito de simplificar as contas e as análises iremos adotar a condição em que o espaçamento angular entre os vórtices do dipolo $\epsilon^{\prime}$ seja muito menor do que a distância entre os dipolos, ou seja, $\epsilon^{\prime} \sim \xi / R \ll 1$.

Os vórtices de um dipolo muito pequeno estão localizados em $\left(\theta_{+}, \phi_{+}\right)=\left(\theta_{0}, \phi_{0}\right)$ e $\left(\theta_{-}, \phi_{-}\right)=\left(\theta_{0}+\epsilon^{\prime}, \phi_{0}\right)$. Logo temos que (observe que não há o fator 2 no denominador de $\chi$ porque a energia de interação provem das velocidades, as quias provem do gradiente de $\chi)$

$$
\chi_{+,-}=\frac{1}{2} \ln \left[1-\cos \left(\theta_{+}\right) \cos \left(\theta_{-}\right)-\sin \left(\theta_{+}\right) \sin \left(\theta_{-}\right) \cos \left(\phi_{+}-\phi_{-}\right)\right] .
$$

Se utilizarmos as seguintes aproximações: $\cos \left(\theta_{0}+\epsilon^{\prime}\right) \approx \cos \left(\theta_{0}\right)+\left[-\sin \left(\theta_{0}\right)\right] \epsilon^{\prime}+\left[-\cos \left(\theta_{0}\right)\right] \frac{\epsilon^{\prime 2}}{2}$ e $\sin \left(\theta_{0}+\epsilon^{\prime}\right) \approx \sin \left(\theta_{0}\right)+\cos \left(\theta_{0}\right) \epsilon^{\prime}+\left(-\sin \left(\theta_{0}\right)\right) \frac{\epsilon^{\prime 2}}{2}$ na Eq. 5.42 para usá-la Eq. 5.26, onde $N=1$, iremos obter a energia de interação de um dipolo de vórtices

$$
E_{i n t, 1}=\frac{2 \pi n \hbar^{2}}{m} \ln \left[\frac{\epsilon^{\prime}}{\sqrt{2}}\right] .
$$

Vamos agora analisar o caso onde há dois pequenos dipolos cujos vórtices têm cargas $q_{1} \operatorname{com} q_{2}$, e $q_{3}$ com $q_{4}$, onde suas coordenadas são: $\left(\theta_{1}, \phi_{1}\right)=\left(\theta_{0}, \phi_{1}\right),\left(\theta_{2}, \phi_{2}\right)=\left(\theta_{0}+\epsilon^{\prime}, \phi_{1}\right)$, $\left(\theta_{3}, \phi_{3}\right)=\left(\theta_{3}, \phi_{3}\right)$ e $\left(\theta_{4}, \phi_{4}\right)=\left(\theta_{3}+\epsilon^{\prime}, \phi_{3}+\delta\right)$, o que configura uma generalização das coordenadas, mas fixando $\epsilon^{\prime}$ como sendo o espaçamento angular entre os vórtices de cada dipolo. No entanto, se adotarmos os eixos de tal forma que o vórtice 1 esteja em $\theta_{1}=0$ e que o vórtice 2 esteja em $\phi=0$ podemos reescrever as coordenadas como: $\left(\theta_{1}, \phi_{1}\right)=(0,0)$, $\left(\theta_{2}, \phi_{2}\right)=\left(\epsilon^{\prime}, 0\right),\left(\theta_{3}, \phi_{3}\right)=\left(\theta_{3}, \phi_{3}\right)$ e $\left(\theta_{4}, \phi_{4}\right)=\left(\theta_{3}+\epsilon, \phi_{3}+\delta\right)$. Veja a Fig. 22

Agora que temos os ângulos podemos calcular os $\chi_{i, j}$ que serão:

$$
\begin{gathered}
\chi_{1,2}=\frac{1}{2} \ln \left[1-\cos \left(\epsilon^{\prime}\right)\right] \\
\chi_{1,3}=\frac{1}{2} \ln \left[1-\cos \left(\theta_{3}\right)\right] \\
\chi_{1,4}=\frac{1}{2} \ln \left[1-\cos \left(\epsilon^{\prime}\right) \cos \left(\theta_{3}\right)+\sin \left(\epsilon^{\prime}\right) \sin \left(\theta_{3}\right)\right] \\
\chi_{2,3}=\frac{1}{2} \ln \left[1-\cos \left(\epsilon^{\prime}\right) \cos \left(\theta_{3}\right)-\sin \left(\epsilon^{\prime}\right) \sin \left(\theta_{3}\right)\right] \\
\chi_{2,4} \frac{1}{2} \ln \left[1-\cos \left(\theta_{3}+\epsilon^{\prime}\right) \cos \left(\epsilon^{\prime}\right)-\sin \left(\epsilon^{\prime}\right) \sin \left(\theta_{3}+\epsilon^{\prime}\right) \cos \left(\phi_{3}+\delta\right)\right] \\
\chi_{3,4}=\frac{1}{2} \ln \left[1-\cos \left(\theta_{3}+\epsilon^{\prime}\right) \cos \left(\theta_{3}\right)-\sin \left(\theta_{3}\right) \sin \left(\theta_{3}+\epsilon^{\prime}\right) \cos (\delta)\right]
\end{gathered}
$$




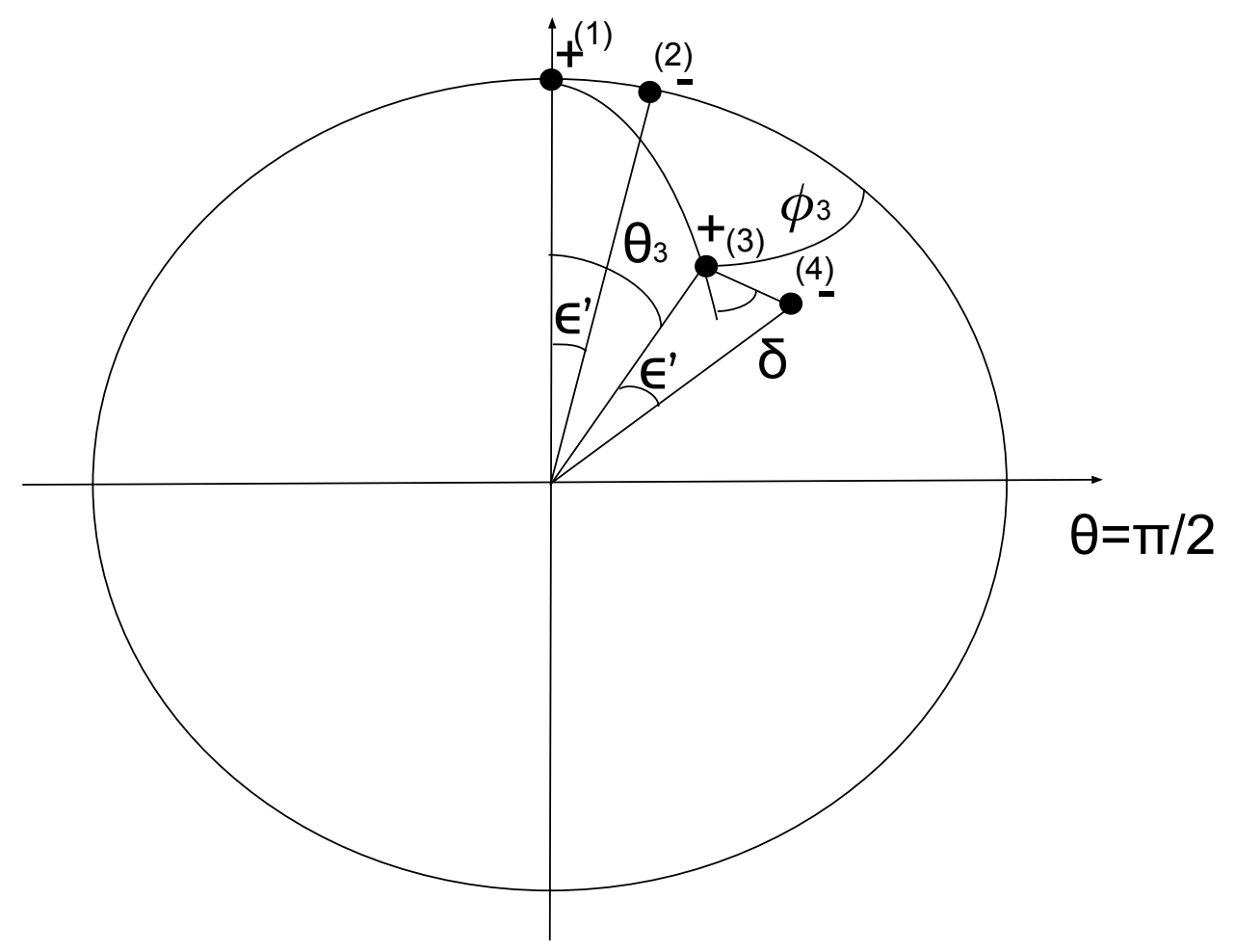

Figura 22: Representação de dipolos alinhados (situação A) em que o primeiro vórtice está localizado em $(\theta, \phi)=(0,0)$. No caso em que o segundo dipolo inverta teremos a situação B.

Fonte: Elaborada pelo autor.

Para o caso $q_{1}=q_{3}=1$ e $q_{2}=q_{4}=-1$ a energia de interação entre os quatro vórtices dos dois dipolos, $E_{i n t, 2}$, será dada pela Eq. 5.26, onde $N=2$,

$$
E_{i n t, 2}=-\frac{2 \pi n \hbar^{2}}{m}\left[-\chi_{1,2}+\chi_{1,3}-\chi_{1,4}-\chi_{2,3}+\chi_{2,4}-\chi_{3,4}\right]
$$

Se substituirmos os valores de $\chi_{i, j}$ na Eq. 5.50 expandindo as funções trigonométricas até a segunda ordem de $\epsilon^{\prime}$ obteremos:

$$
E_{i n t, 2} \approx \frac{4 \pi n \hbar^{2}}{m} \ln \left[\frac{\epsilon^{\prime}}{\sqrt{2}}\right]-\frac{\pi n \hbar^{2}}{m} \ln \left[1+\frac{\epsilon^{\prime} \cos \left(\phi_{3}\right)}{1-\cos \left(\theta_{3}\right)}+\frac{\delta \epsilon^{\prime}}{1-\cos \left(\theta_{3}\right)} \sin \left(\theta_{3}\right) \sin \left(\phi_{3}\right)\right] .
$$

Se utilizarmos a aproximação $\ln [1+\tilde{\chi}] \approx \tilde{\chi}$ quando $\tilde{\chi} \ll 1$ podemos reescrever a Eq. 5.51 como sendo

$$
E_{i n t, 2} \approx \frac{4 \pi n \hbar^{2}}{m} \ln \left[\frac{\epsilon^{\prime}}{\sqrt{2}}\right]-\frac{\pi n \hbar^{2}}{m}\left[\frac{\cos \left(\phi_{3}\right)}{1-\cos \left(\theta_{3}\right)} \epsilon^{\prime 2}+\frac{\sin \left(\theta_{3}\right) \sin \left(\phi_{3}\right)}{1-\cos \left(\theta_{3}\right)} \epsilon^{\prime} \delta\right] .
$$

O primeiro termo da Eq. 5.52 é o dobro da Eq. 5.43 porque este termo é atribuído a energia de interação entre os vórtices do dipolo (é o dobro porque neste caso há dois dipolos). Então o segundo termo da Eq. 5.52 é a energia de interação dipolo-dipolo. Se considerarmos $\theta_{3} \gg \epsilon^{\prime}$, ou seja, assumirmos que o espaçamento angular entre os vórtices positivos é muito maior que o espaçamento angular de dipolo temos que $\left|4 \ln \frac{\epsilon^{\prime}}{\sqrt{2}}\right| \gg$ 
$\left|-\left[\frac{\cos \left(\phi_{3}\right)}{1-\cos \left(\theta_{3}\right)} \epsilon^{\prime 2}+\frac{\sin \left(\theta_{3}\right) \sin \left(\phi_{3}\right)}{1-\cos \left(\theta_{3}\right)} \epsilon^{\prime} \delta\right]\right|$, pois como $\epsilon^{\prime} \ll 1$ o logarítmo vai divergir para $-\infty$ muito rapidamente enquanto o outro termo converge para zero. Então a energia de interação dipolo-dipolo interfere muito pouco na energia de interação do sistema. A energia de interação dipolo-dipolo contribui pouco na energia do sistema porque o módulo da velocidade de fluxo depende da cotangente da metade do ângulo entre os vórtices (ver Eq. 5.27), então os vórtices irão interagir mais intensamente com seus vizinhos mais próximos.

Se desprezarmos a interaçao dipolo-dipolo os dipolos irão transladar pela esfera em eixos que dependerão das condições iniciais com velocidades de translação dadas pela Eq. 5.28 para $\delta \theta=\epsilon^{\prime} / 2$. No entanto, se houver choque entre os dipolos a condição $\theta_{3} \gg \epsilon^{\prime}$ deixa de ser válida quebrando assim esse movimento de traslação. 


\section{CONCLUSÃO}

No presente projeto estudamos a formação de um condensado de Bose-Einstein em uma casca esférica quase-2D. Vimos a possibilidade de termos um valor finito para a temperatura crítica de condensação, mesmo no limite em que a espessura da casca vai a zero. A temperatura foi calculada numericamente, considerando diferentes geometrias e volumes para o aprisionamento dos átomos. Os resultados foram comparados à solução semi-clássica, cujo regime de validade foi justificado com base no teorema de Weyl.

Na segunda etapa consideramos a dinâmica e estabilidade de excitações de vórtices do condensado na casca esférica. A topologia desse sistema já impõe um vínculo sobre a configuração dos vórtices, a qual consistirá de pares de vórtice e anti-vórtices de mesma carga (circulação oposta). Calculamos o campo de velocidade para configurações arbitrária de pares, e através dele a dinâmica e energia dos vórtices. Usando a aproximação de dipolo pudemos analisar como a energia do sistema varia com o alinhamento dos dipolos, além de prever a dinâmica dos pares individuais, com base no balanço das auto-energias e da energia de interação entre os dipolos. Futuramente pretendemos realizar simulações numéricas para acompanhar a dinâmica das configurações de pares e dos dipolos de vórtices consideradas nesse projeto.

Finalmente, vale ressaltar aqui que a configuração estudada, isto é, o sistema do gás 2D uniformente distribuido na casca esférica, poderá ser analisada experimentalmente em um futuro próximo em vista da possibilidade de um experimento com bubble trap ser conduzido em um ambiente de microgravidade ${ }^{53}$. Em um trabalho ainda não publicado conduzido por colaboradores da Universidade de Paris XVIII, um experimento com o potencial de bubble trap foi realizado, e uma transição de um condensado com uma rede de vórtices para uma estrutura anular sem vórtices e um único vórtice gigante central foi observada. No entanto, ainda não se conhecem justificativas teóricas que expliquem o fenômeno observado no contexto desse potencial. Futuramente pretendemos fornecer explicações teóricas para os resultados experimentais obtidos pela colaboração, no projeto USP - COFECUB (19.1.411.1.9). Para tanto, pretendemos adaptar os nossos resultados e técnicas para o bubble trap considerendo o efeito do potencial gravitacional ${ }^{53}$. 



\section{REFERÊNCIAS}

1 PETHICK, C.; SMITH, H. Bose-Eintein condensation in dilute gases. 2nd ed. Cambrigde: Cambridge University Press, 2008. 569 p. ISBN 978-0521846516.

2 DAHMEN, S. Einstein e a teoria quântica de gases. Revista Brasileira de Ensino de Física, v. 27, p. 463-512, 2005. doi: 10.1590.

3 KETTERLE, W. et al. Making, probing and understanding Bose-Einstein condensates. Disponível em: https://arxiv.org/pdf/cond-mat/9904034v2.pdf. Acesso em: 22 nov. 2019.

4 ZOBAY, O.; GARRAWAY, B. M. Two-dimensional atom trapping in field-induced adiabatic potentials. Physical Review Letters, v. 86, p. 1195-1198, 2001. doi: 10.1103/PhysRevLett.86.1195.

5 ZOBAY O.; GARRAWAY, B. Atom trapping and two-dimensional Bose-Einstein condensates in field-induced adiabatic potentials. Physical Review A, v. 69, p. 023605, 2004. doi: 10.1103/PhysRevA.69.02360.

6 HAMMING, R. W. Numerical methods for scientists and engineers. 2nd ed. Nova York: Dover Publications, 1973. 752 p. ISBN 9780486652412.

7 BAGNATO, V. et al. Bose-Einstein condensation in an external potential. Physical Review A, v. 35, n. 10, p. 4354-4358, 1987. doi: 10.1103/PhysRevA.35.4354.

8 YUKALOV, V. I. Modified semiclassical approximation for trapped Bose gases. Physical Review A, v. 72, p. 033608, 2005. doi: 10.1103/PhysRevA.72.033608.

9 TURNER, A. M.; VITELLI, V.; NELSON, D. R. Vortices on curved surfaces. Review Modern Physics, v. 82, p. 1301-1348, 2010. doi: 10.1103/RevModPhys.82.1301.

10 OVRUT, B. A.; THOMAS, S. Theory of vortices and monopoles on a sphere. Physical Review D, v. 43, p. 1314-1322, 1991. doi: 10.1103/PhysRevD.43.1314.

11 REUTHER S.; VOIGT, A. The interplay of curvature and vortices in flow on curved surfaces. Multiscale Modeling and Simulation, v. 13, p. 632-643, 2015. doi: 10.1137/140971798.

12 MASSIGNAN P.; FETTER, A. L. Superfluid vortex dynamics on planar sectors and cones. Physical Review A, v. 99, p. 063602, 2019. doi: 10.1103/PhysRevA.99.063602.

13 BUTKOV, E. Física matemática. Rio de Janeiro: Editora Guanabara Dois S.A., 1978. 725 p. ISBN 9788521611455.

14 BAEK, S. K. Vortex interaction on curved surfaces. Physical Review E, v. 86, p. 056603, 2012. doi: 10.1103/PhysRevE.86.056603.

15 NEELY, T. W. et al. Observation of vortex dipoles in an oblate BoseEinstein condensate. Physical Review Letters, v. 104, p. 160401, 2010. doi: 10.1103/PhysRevLett.104.160401. 
16 BOSE, S.; THEIMER, O. The beginning of quantum statistics: a translation of "Planck's law and the light quantum hypothesis". American Journal of Physics, v. 44, p. 1056-1057, 1976. doi: 10.1119/1.10584.

17 BOSE, S. Planck's law and light quantum hypothesis. Zeitschrift für Physik, v. 26, p. 178-181, 1924. ISSN 0044-3328. doi: 10.1007/BF01327326. Disponível em: http://web.ihep.su/dbserv/compas/src/bose24/eng.pdf. Acesso em: 17 fev. 2020.

18 BLANPIED, W. A. Satyendranath Bose: Co-founder of quantum statistics. American Journal of Physics, v. 40, p. 1056-1057. doi: 10.1119/1.1986805, 1972.

19 LEGGETT, A. J. Quantum liquids: Bose condensation and Cooper pairing in condensed-matter systems. New York: Oxford University Press., 2006. 237 p. ISBN 9780198526438 .

20 UEDA, M. Fundamentals and new frontiers of Bose-Einstein condensation. New York: World Scientific, 2010. Disponível em: https://doi.org/10.1142/7216. Acesso em: 25 nov. 2019. 368 p. ISBN 9789812839596.

21 ANDERSON, M. H. et al. Observation of Bose-Einstein condensation in a dilute atomic vapor. Science, v. 269, n. 4, p. 198-201, 1955. doi: 10.1126/science.269.5221.198.

22 BRADley, C. C.; SACKETT, C. A.; TOlletT, J. J.; Hulet, R. G. Evidence of Bose-Einstein condensation in an atomic gas with attractive interactions. Physical Review Letters, v. 75, p. 1687-1690, 1995. doi: 10.1103/PhysRevLett.75.1687. Disponível em: https://link.aps.org/doi/10.1103/PhysRevLett.75.1687. Acesso 22 nov. 2019.

23 DAVIS, K. et al. Bose-Einstein condensation in a gas of sodium atoms. Physical Review Letters, v. 75, p. 3969-, 1995. doi: 10.1109/EQEC.1996.561567.

24 GARRAWAY B. M.; PERRIN, H. Recent developments in trapping and manipulation of atoms with adiabatic potentials. Journal of Physics B: atomic, molecular and optical physics, v. 69, p. 023605, 2016. Disponível em: https://iopscience.iop.org/article/10.1088/0953-4075/49/17/172001. Acesso em: 22 nov. 2019 .

25 ELLIOT, E. R. et al. Nasa's cold atom lab (cal): system development and ground test status. npjMicrogravity, v. 4, p. 1-7, 2018. doi: 10.1038/s41526-018-0049-9.

26 LUNDBLAD, N. et al. Shell potentials for microgravity Bose-Einstein condensates. 2019. Disponível em: https://arxiv.org/abs/1906.05885. Acesso em: 22 nov. 2019 .

27 PITAEVSKII, L. P. Vortex lines in an imperfect Bose gas. Journal of Experimental and Theoretical Physics, v. 13, p. 451-454, 1961. Disponível em: http://www.jetp.ac.ru/cgi-bin/e/index/e/13/2/p451?a=list. Acesso em: 22 nov. 2019.

28 SALINAS, S. R. A. Introdução a física estatística. 2. ed. São Paulo: Edusp, 2005. ISBN 9788521611455.

29 COOK G.; DICKERSON, R. H. Understanding the chemical potential. American Journal of Physics, v. 63, 1995. doi: 10.1119/1.17844. 
30 MUlHALL D.; MOELTERB, M. J. Calculating and visualizing the density of state for simple quantum mechanical systems. American Journal of Physics, v. 82, p. 665, 2014. doi: 10.1119/1.4867489.

31 GRIFFITHS D. J.; SCHROETER, D. F. Introduction to quantum mechanics. 2nd ed. Cambrigde: Cambridge University Press., 2018. 360 p. ISBN 1107189632.

32 GROSSMAN S.; HOLTHAUS, M. On Bose-Einstein condensation in harmonic traps. Physics Letters A, v. 208, p. 177-260, 1995. Disponível em: https://www.sciencedirect.com/journal/physics-letters-a/vol/208/issue/3. Acesso em: 22 nov. 2019.

33 SCHARF, G. On Bose-Einstein condensation. American Journal of Physics, v. 61 , p. 842-845, 1993. doi: 10.1119/1.17416.

34 DAlFOVO, F.; GIORGINI, S.; PITAEVSKII, L. P.; STRINGARI, S. Theory of Bose-Einstein condensation in trapped gases. Review Modern Physics, v. 71, p. 463-512, 1999. doi: 10.1103/RevModPhys.71.463.

35 WEYL, H. Das asymptotische verteilungsgesetz der eigenwerte linearer partieller differentialgleichungen (mit einer anwendung auf die theorie der hohlraumstrahlung). Mathematische Annalen, v. 71, n. 4, p. 441-479, 1912. doi: 10.1007/BF01456804. ISSN 1432-1807.

36 BERETA, S. J.; MADEIRA, L.; BAGNATO, V. S.; CARACANHAS, M. A. Bose-Einstein condensation in spherically symmetric traps. American Journal of Physics, v. 87, 2019. doi: 10.1119/1.5125092.

37 ABRAMOWITZ, M. Handbook of mathematical functions: with formulas, graphs and mathematical tables. 2nd ed. Nova York: Dover Books on Mathematics, 2012. 1048 p. ISBN 9788521611455.

38 TONONI, A.; SALASNICH, L. Bose-Einstein condensation on the surface of a sphere. Physical Review Letters, v. 123, p. 160403, 2019. doi: 10.1103/PhysRevLett.123.160403.

39 MCGEE S. A.; HOLLAND, M. J. Rotational dynamics of vortices in confined Bose-Einstein condensates. Physical Review A, v. 63, p. 043608, 2001. doi: 10.1103/PhysRevA.63.043608.

40 GROSS, E. P. Structure of a quantized vortex in boron Systems.

Il Nuevo Cimento, v. 20, p. 454-477, 1961. Disponível em: https://link.springer.com/content/pdf/10.1007\%2FBF02731494.pdf. Acesso em: 25 nov. 2019.

41 FETTER, A. L. Rotating trapped Bose-Einstein condensates. Laser Physics, v. 18, n. 1, p. 1-11, 2008. doi: 10.1134/S1054660X08010015. ISSN 1555-6611.

42 SHEEHY, D. E.; RADZIHOVSKY, L. Vortex lattice inhomogeneity in spatially inhomogeneous superfluids. Physical Review A, v. 70, p. 051602, 2004. doi: 10.1103/PhysRevA.70.051602.

43 FETTER, A. L. Vortices and dynamics in trapped Bose-Einstein condensates. Journal of Low Temperature Physics, v. 161, n. 5, p. 445-459, 2010. doi: 10.1007/s10909-010-0202-7. ISSN 1573-7357. 
44 ABO-SHAEER, J. R. et al. Observation of vortex lattices in Bose-Einstein condensates. Science, v. 292, p. 476-479, 2001. doi: 10.1126/science.1060182.

45 FETTER A.; KIM, J. Vortex precession in a rotating nonaxisymmetric trapped Bose-Einstein condensate. Journal of Low Temperature Physics, v. 125, p. 239-248, 2001. doi: 10.1023/A:1012919924475.

46 SVIDZINSKY A. A.; FETTER, A. L. Dynamics of a vortex in a trapped Bose-Einstein condensate. Physical Review A, v. 62, p. 063617, 2000. doi: 10.1103/PhysRevA.62.063617.

47 FETTER L. A.; SVIDZINSKY, A. A. Vortices in a trapped dilute Bose-Einstein condensate. Journal of Physics: condensed matter, v. 13, n. 12, p. R135-R194, 2001. doi: 10.1088/0953-8984/13/12/201.

48 FETTER A.; SVIDZINSKY, A. Topical review: vortices in a trapped dilute Bose-Einstein condensate. Journal of Physics, v. 13, 2001. doi: 10.1088/0953$8984 / 13 / 12 / 201$.

49 SVIDZINSKY A.; FETTER, A. L. Stability of a vortex in a trapped BoseEinstein condensate. Physical Review Letters, v. 84, p. 5919-23, 2000. doi: 10.1103/PhysRevLett.84.5919.

50 FETTER, A. L. Vortices in an imperfect Bose gas. iv. translational velocity. Physycal Review, v. 151, p. 100-104, 1966. doi: 10.1103/PhysRev.151.100.

51 CALDERARO, L. et al. Vortex dynamics in coherently coupled Bose-Einstein condensates. Physycal Review A, v. 95, p. 023605, 2017. doi: 10.1103/PhysRevA.95.023605.

52 GUENTHER, N.; MASSIGNAN, P.; FETTER, A. L. Quantized superfluid vortex dynamics on cylindrical surfaces and planar annuli. Physical Review A, v. 96, p. 063608, 2017. doi: 10.1103/PhysRevA.96.063608.

53 SUN, K. et al. Static and dynamic properties of shell-shaped condensates. Physical Review A, v. 98, p. 013609, 2018. doi: 10.1103/PhysRevA.98.013609. 
Apêndices 



\section{APÊNDICE A - DEMONSTRAÇÃO DO RESULTADO DA INTEGRAL UTILIZADO PARA CONCLUIR A EQ. 2.3}

Neste apêndice iremos demonstrar o resultado da integral utilizado para concluir a Eq. 2.3. O princípio de Heisenberg diz que o menor volume no espaço de momento e real é:

$$
d V=d p^{3} d r^{3} / h^{3}
$$

onde $d p$ é o diferencial de momento e $d r$, de espaço. Se quizermos saber todos os estados possíveis até certo $p^{\prime}$, ou seja, o cumulante $G(p)$, devemos integrar $d V$ sobre 0 até $p^{\prime}$ em todo o volume confinado. Se considerarmos $d^{3} p=4 \pi p^{2} d p$, o cumulante ficará:

$$
G\left(p^{\prime}\right)=\frac{1}{h^{3}} \int d^{3} r \int_{0}^{p^{\prime}} 4 \pi p^{2} d p=\frac{4 \pi}{3 h^{3}} \int d^{3} r p^{\prime 3} .
$$

A energia $\epsilon$ corresponde a parte potencial mais a cinética, $\epsilon=\frac{p^{\prime 2}}{2 m}+U(\mathbf{r})$. Logo $p^{\prime}=2 m(\epsilon-U(\mathbf{r}))^{1 / 2}$, isso faz com que a Eq. A.2 fique da forma:

$$
G(\epsilon)=\frac{4 \pi(2 m)^{3 / 2}}{3 h^{3}} \int_{V}(\epsilon-U(\mathbf{r}))^{3 / 2} d^{3} r .
$$

Se o cumulante nos dá o total de estados até $\epsilon$ então a derivada do cumulante será o número de estados entre $\epsilon+d \epsilon$ que corresponde ao $g(\epsilon)$. Logo temos que:

$$
g(\epsilon)=\frac{2 \pi(2 m)^{3 / 2}}{h^{3}} \int_{V(\epsilon)} \sqrt{\epsilon-U(\mathbf{r})} d^{3} r .
$$





\section{APÊNDICE B - DENSIDADE DE ESTADOS DO OSCILADOR HARMÔNICO}

Para calcular a densidade de estados do oscilador harmônico precisamos resolver o problema: quantas vezes é possível adicionar três números para obter um número específico $n$ ? Por exemplo, supondo que queiramos obter o número $n=2$, o qual é igual a $0+0+2$, ou $0+1+1$, ou $0+2+0$, ou $1+1+0$, ou $2+0+0$, ou $1+0+1$, teremos seis combinações diferentes para obter esse número. A resposta para esse problema é a combinação de $n+2$ com 2, $C_{n+2,2}=\frac{(n+2) !}{n ! 2 !}$. De acordo com o exemplo dado $(n=2) C_{2+2,2}=6$. É possível ver que a resposta para este problema é a combinação quando consideramos um unidade representada pelo símbolo * o qual será permutado com dois sinais de adição + , por exemplo, se tivermos $n=3$, as possibilidades serão: $++^{* * *}$, ou $+^{*}+{ }^{* *}$, ou $+^{* *}+{ }^{*} \ldots$, neste caso nós temos $C_{3+2,2}=15$ combinações.

Então assumindo $n=n_{1}+n_{2}+n_{3}$ nós agora sabemos quantas maneiras diferentes podemos obter a energia de nível $\varepsilon=\hbar w\left(n+\frac{1}{2}\right)$. A total de combinações possíveis $G(n)$ para obter até um nível de energia de estado $n$ será

$$
\sum_{i=0}^{n} \frac{(i+2) !}{i ! 2 !}=\sum_{i=0}^{n} \frac{i^{2}+3 i+2}{2}
$$

com um pouco de álgebra

$$
C(n)=\frac{5}{6}+\frac{4}{3} n+\frac{n^{2}}{2}+\frac{(n+1)^{3}}{6} .
$$

A derivação com respeito a $n$ para obter a densidade de estados $g_{o s c}(n)$ :

$$
g_{\text {osc }}(\varepsilon) d n=\left(\frac{11}{6}+2 n+\frac{n^{2}}{2}\right) d n .
$$

Após a substituição $n=\frac{\varepsilon}{\hbar w}-\frac{1}{2}$ finalmente temos a densidade de estados

$$
g_{o s c}(\varepsilon)=\frac{1}{2} \frac{\varepsilon^{2}}{(\hbar w)^{3}}+\frac{3}{2} \frac{\varepsilon}{(\hbar w)^{2}}+\frac{23}{24} .
$$





\section{APÊNDICE C - DENSIDADE DE ESTADOS DO OSCILADOR HARMÔNICO UTILIZANDO A EQ. 2.3}

Dada a energia potencial de interesse $U=\frac{m w^{2}}{2}\left(x^{2}+y^{2}+z^{2}\right)$. Aplicando este potencial na Eq. 2.3 resulta

$$
g_{\text {osc }}(\varepsilon)=\frac{1}{4 \pi^{2}}\left(\frac{2 m}{\hbar^{2}}\right)^{\frac{3}{2}} \int_{V} d^{3} r \sqrt{\varepsilon-\left[\frac{m w^{2}}{2}\left(x^{2}+y^{2}+z^{2}\right)\right]} .
$$

Sendo $d^{3} r=r^{2} \sin \theta d \theta d \phi d r$.

Para facilitar a resoluçao da integral vamos utilizar as seguintes substituição de variáveis: $\varepsilon_{1}=\frac{m w^{2}}{2}$ e $R=\sqrt{\frac{\varepsilon_{1}}{\varepsilon}} r$. O limite da integral será quando a energia for puramente potencial, e isso ocorrerá quando $r_{m a ́ x}^{2}=\frac{\varepsilon}{\varepsilon_{1}}$, na nossa substituição de variável será o mesmo que $R=1$. Dessa forma a Eq. C.1 ficará da forma

$$
g_{\text {osc }}(\varepsilon)=\frac{1}{4 \pi^{2}}\left(\frac{2 m}{\hbar^{2}}\right)^{\frac{3}{2}} 4 \pi \frac{\varepsilon^{2}}{\varepsilon_{1}^{\frac{3}{2}}} \int_{0}^{1} R^{2} \sqrt{1-R^{2}} d R .
$$

Dado valor da $\int_{0}^{1} R^{2} \sqrt{1-R^{2}} d R=\frac{\pi}{16}$. Fazendo algumas simplificações, e retornando a transformação de variável $\varepsilon_{1}=\frac{m w^{2}}{2}$, temos que

$$
g_{\text {osc }}(\varepsilon)=\frac{\varepsilon^{2}}{2(\hbar w)^{3}}
$$





\section{APÊNDICE D - DEMONSTRAÇÃO DO RESULTADO DA INTEGRAL UTILIZADO PARA CONCLUIR A EQ. 2.16}

Neste apêndice iremos demonstrar o resultado da integral utilizado para concluir a Eq. 2.16.

$$
\int_{0}^{\infty} \epsilon^{\alpha-1} \frac{1}{e^{\beta \epsilon}-1} d \epsilon
$$

O primeiro passo para a resolução desta integral é fazer a transformação de variável:

$$
\beta \epsilon=x,
$$

o que nos dá:

$$
\frac{1}{\beta^{\alpha}} \int x^{\alpha-1} \frac{d x}{e^{x}-1}
$$

- O segundo passo é aplicar a expansão:

$$
\frac{1}{e^{x}-1}=\sum_{n=1}^{\infty} e^{-n x}
$$

Assim a integral ficará:

$$
\frac{1}{\beta^{\alpha}} \sum_{n=1}^{\infty} \int e^{-n x} x^{\alpha-1} d x
$$

O terceiro passo é fazer uma nova substituição:

$$
n x=X
$$

Com essa nova substituição a integral ficará:

$$
\frac{1}{\beta^{\alpha}} \sum_{n=1}^{\infty} n^{-\alpha} \int_{0}^{\infty} X^{\alpha-1} e^{-X} d X
$$

Sendo $\beta=1 / k_{B} T, \Gamma(\alpha)=\int_{0}^{\infty} X^{\alpha-1} e^{-X} d X$ e $\zeta(\alpha)=\sum_{n=1}^{\infty} n^{-\alpha}$. 



\section{APÊNDICE E - DEMONSTRAÇÃO DAS EQ. 2.22 E 2.24}

Neste apêndice iremos demonstrar a Eq. 2.22 e 2.24 através da Eq. 2.20.

Considere a equação de Schrödinger abaixo:

$$
\frac{-\hbar^{2}}{2 m} \nabla^{2} \Psi(r, \theta, \phi)+U(\mathbf{r}) \Psi(r, \theta, \phi)=\epsilon \Psi(r, \theta, \phi)
$$

Se considerarmos que $U(r)=0$ e que $\epsilon=\frac{\hbar^{2} k^{2}}{2 m}$ a Eq. E.1 fica da forma:

$$
\nabla^{2} \Psi_{k}+k^{2} \Psi_{k}=0
$$

Se fizermos a transformação $\Psi(r, \theta, \phi)=R(r) Y_{l, m}(\theta, \phi)$ e abrirmos o laplaciano a Eq. E.2 fica da seguinte forma:

$$
\begin{aligned}
0 & =\left[\frac{1}{r^{2}} \frac{\partial}{\partial r}\left(r^{2} \frac{\partial}{\partial r}\right)+\frac{1}{r^{2} \sin (\theta)} \frac{\partial}{\partial \theta}\left(\sin (\theta) \frac{\partial}{\partial \theta}\right)\right. \\
& \left.+\frac{1}{r^{2} \sin ^{2} \theta} \frac{\partial^{2}}{\partial \phi^{2}}\right] R(r) Y_{l, m}(\theta, \phi)+k^{2} R(r) Y_{l, m}(\theta, \phi) .
\end{aligned}
$$

Sabemos que a derivada de $R(r)$ com respeito a $\theta$ e $\phi$ é zero e que a derivada de $Y_{l, m}$ com respeito a $r$ também é zero. Se multiplicarmos a Eq. E.3 por $r^{2} /\left(R(r) Y_{l, m}(\theta, \phi)\right)$ ela ficará da forma:

$$
\begin{aligned}
0 & =\frac{1}{R(r)} \frac{\partial}{\partial r}\left(r^{2} \frac{\partial R(r)}{\partial r}\right)+\frac{1}{Y_{l, m}(\theta, \phi) \sin (\theta)} \frac{\partial\left(\sin (\theta) \frac{\partial Y_{l, m}(\theta, \phi)}{\partial \theta}\right)}{\partial \theta} \\
& +\frac{1}{Y_{l, m}(\theta, \phi) \sin ^{2}(\theta)} \frac{\partial^{2} Y_{l, m}(\theta, \phi)}{\partial \phi^{2}}+k^{2} r^{2}
\end{aligned}
$$

Perceba que o primeiro e o quarto termos são somente radiais e o segundo e o terceiro termos são angulares. Vamos assumir que a parte angular é igual a $-l(l+1)$ onde $l$ é um número inteiro positivo. Assim sendo podemos separar a Eq. E.3 em duas:

$$
\begin{gathered}
l(l+1)=\frac{1}{R(r)} \frac{\partial}{\partial r}\left(r^{2} \frac{\partial R(r)}{\partial r}\right)+k^{2} r^{2}, \\
-l(l+1)=\frac{1}{Y_{l, m}(\theta, \phi) \sin (\theta)} \frac{\partial\left(\sin (\theta) \frac{\partial Y_{l, m}(\theta, \phi)}{\partial \theta}\right)}{\partial \theta}+\frac{1}{Y_{l, m}(\theta, \phi) \sin ^{2}(\theta)} \frac{\partial^{2} Y_{l, m}(\theta, \phi)}{\partial \phi^{2}} .
\end{gathered}
$$


Se multiplicarmos a Eq. E.4 por $Y_{l, m}$ teremos a Eq. 2.24.

$$
\frac{1}{\sin \theta} \frac{\partial\left(\sin \theta \frac{\partial Y_{l}, m}{\partial \theta}\right)}{\partial \theta}+\frac{1}{\sin ^{2} \theta} \frac{\partial^{2} Y_{l, m}}{\partial \varphi^{2}}+l(l+1) Y_{l, m}=0 .
$$

Se dividirmos a Eq. E.4 por $r^{2}$ e aplicarmos a regra da cadeia em no primeiro termo depois da igualdade teremos a Eq. 2.22,

$$
\frac{\mathrm{d}^{2} R_{n, l}}{\mathrm{~d} r^{2}}+\frac{2}{r} \frac{\mathrm{d} R_{n, l}}{\mathrm{~d} r}+\left(k^{2}-\frac{l(l+1)}{r^{2}}\right) R_{n, l}=0 .
$$

Perceba que a derivada parcial será igual a derivada total em relação a $r$, pois $R(r)$ não depende de $\theta$ ou $\phi$. 


\section{APÊNDICE F - DEDUÇÃO DA EQ. 2.48}

Esse apêndice propõe a dedução da Eq. 2.48.

De forma análoga ao argumentado no apêndice A. Temos que:

$$
d A=\frac{d^{2} p d^{2} r}{h^{2}}
$$

então o cumulante em duas dimensões $G_{2 D}(\epsilon)$ será:

$$
G_{2 D}(\epsilon)=\frac{1}{h^{2}} \int d^{2} p d^{2} r=\frac{1}{h^{2}} \pi \int p^{\prime 2} d^{2} r
$$

se substituirmos ${p^{\prime}}^{2}=(\epsilon-U(\mathbf{r})) 2 m$ e assumirmos que $U(\mathbf{r})=0$ o cumulante fica da forma:

$$
G_{2 D}(\epsilon)=\frac{1}{h^{2}} \pi \int 2 m \epsilon d^{2} r=2 \pi m \frac{A}{h^{2}} \epsilon,
$$

tomando a derivada e susbtituindo $\hbar=h / 2 \pi$ temos:

$$
g_{2 D}(\epsilon)=\frac{m}{2 \pi} \frac{A}{\hbar^{2}}
$$





\section{APÊNDICE G - DEDUÇÃO DA EQ. 5.13}

Neste apêndice deduziremos a Eq. 5.13 separando a parte real e a imaginária de $F=\ln \left(\frac{2 R \tan (\theta / 2) e^{i \phi}-z_{+}}{\tan (\theta / 2) e^{i \phi}-z_{-}}\right)$, onde $z_{ \pm}=\tan \left(\theta_{ \pm} / 2\right) e^{i \phi_{ \pm}}$. Se subtituirmos $z_{ \pm}$em $F$ temos:

$$
F=\ln \left(\frac{\tan (\theta / 2) e^{i \phi}-z_{+}}{\tan (\theta / 2) e^{i \phi}-z_{-}}\right)
$$

Agora se substituirmos $z_{ \pm}=2 R \tan \left(\theta_{ \pm} / 2\right) e^{i \phi_{ \pm}}$obteremos

$$
\begin{gathered}
F=\ln \left(\frac{2 R \tan (\theta / 2) e^{i \phi}-\tan \left(\theta_{+} / 2\right) e^{i \phi_{+}}}{2 R \tan (\theta / 2) e^{i \phi}-\tan \left(\theta_{-} / 2\right) e^{i \phi_{-}}}\right), \\
F=\ln \left[\tan (\theta / 2) e^{i \phi}-\tan \left(\theta_{+} / 2\right) e^{i \phi_{+}}\right]-\ln \left[\tan (\theta / 2) e^{i \phi}-\tan \left(\theta_{-} / 2\right) e^{i \phi_{-}}\right],
\end{gathered}
$$

O primeiro termos a direita da igualdade da Eq. G.3 é analogo ao segundo termo por isso vamos calcular apenas a parte real do primeiro termo. Se fizermos a substituição $e^{i \phi_{+}}=\cos \phi_{+}+i \sin \phi_{+}$obteremos:

$$
\ln \left[\tan (\theta / 2)(\cos \phi+i \sin \phi)-\tan \left(\theta_{+} / 2\right)\left(\cos \phi_{+}+i \sin \phi_{+}\right)\right]
$$

$$
\ln \left\{\tan (\theta / 2) \cos \phi-\tan \left(\theta_{+} / 2\right) \cos \phi_{+}+i\left[\tan (\theta / 2) \sin \phi-\tan \left(\theta_{+} / 2\right) \sin \phi_{+}\right]\right\}
$$

Para simplificar a expressão vamos definir duas novas variáveis $u=[\tan (\theta / 2) \cos \phi-$ $\left.\tan \left(\theta_{+} / 2\right) \cos \phi_{+}\right]$e $v=\left[\tan (\theta / 2) \sin \phi-\tan \left(\theta_{+} / 2\right) \sin \phi_{+}\right]$. Portanto podemos reescrever a Eq. G.5 na forma:

$$
\ln (u+i v)=\ln \left[\sqrt{u^{2}+v^{2}}\left(\frac{u}{\sqrt{u^{2}+v^{2}}}+i \frac{v}{\sqrt{u^{2}+v^{2}}}\right)\right]=\ln \left[\sqrt{u^{2}+v^{2}}\left(e^{i \arctan (v / u)}\right)\right] .
$$

Da Eq. G.6 podemos concluir:

$$
\ln \left[\sqrt{u^{2}+v^{2}}\left(e^{i \arctan (v / u)}\right)\right]=\ln \sqrt{u^{2}+v^{2}}+i \arctan v / u,
$$

ou seja, parte real é $\ln \sqrt{u^{2}+v^{2}}$. Se recuperarmos $u$ e $v$ obteremos:

$$
\frac{1}{2} \ln \left[\tan ^{2}(\theta / 2)+\tan ^{2}\left(\theta_{+} / 2\right)-2 \tan (\theta / 2) \tan \left(\theta_{+} / 2\right)\left(\cos \phi \cos \phi_{+}+\sin \phi \sin \phi_{+}\right)\right]
$$

De forma análoga, o negativo:

$\frac{1}{2} \ln \left[\tan ^{2}(\theta / 2)+\tan ^{2}\left(\theta_{-} / 2\right)-2 \tan (\theta / 2) \tan \left(\theta_{-} / 2\right)\left(\cos \phi \cos \phi_{-}+\sin \phi \sin \phi_{-}\right)\right]$ 
Portanto temos que $\chi_{+}-\chi_{-}$será:

$$
\ln \frac{\left[\tan ^{2}(\theta / 2)+\tan ^{2}\left(\theta_{+} / 2\right)-2 \tan (\theta / 2) \tan \left(\theta_{+} / 2\right)\left(\cos \phi \cos \phi_{+}+\sin \phi \sin \phi_{+}\right)\right]}{\left[\tan ^{2}(\theta / 2)+\tan ^{2}\left(\theta_{-} / 2\right)-2 \tan (\theta / 2) \tan \left(\theta_{-} / 2\right)\left(\cos \phi \cos \phi_{-}+\sin \phi \sin \phi_{-}\right)\right]}
$$

Lembrando que a Eq. 5.13 é a parte real da Eq. 5.12.

Podemos reescrever a Eq. 5.12 na forma:

$$
\chi_{s}=\chi_{+}-\chi_{-},
$$

onde

$$
\chi_{+}(\theta, \phi)=\frac{1}{2} \ln \left[\tan ^{2}(\theta / 2)+\tan ^{2}\left(\theta_{+} / 2\right)-2 \tan (\theta / 2) \tan \theta_{+} / 2 \cos \left(\phi-\phi_{+}\right)\right],
$$

e

$$
\chi_{-}(\theta, \phi)=\frac{1}{2} \ln \left[\tan ^{2}(\theta / 2)+\tan ^{2}\left(\theta_{-} / 2\right)-2 \tan (\theta / 2) \tan \left(\theta_{-} / 2\right) \cos \left(\phi-\phi_{-}\right)\right] .
$$

